\title{
HYDRODYNAMIC LIMIT OF A KINETIC GAS FLOW PAST AN OBSTACLE
}

\author{
R. ESPOSITO, Y. GUO, AND R. MARRA
}

\begin{abstract}
Given an obstacle in $\mathbb{R}^{3}$ and a non-zero velocity with small amplitude at the infinity, we construct the unique steady Boltzmann solution flowing around such an obstacle with the prescribed velocity as $|x| \rightarrow \infty$, which approaches the corresponding Navier-Stokes steady flow, as the mean-free path goes to zero. Furthermore, we establish the error estimate between the Boltzmann solution and its Navier-Stokes approximation. Our method consists of new $L^{6}$ and $L^{3}$ estimates in the unbounded exterior domain, as well as an iterative scheme preserving the positivity of the distribution function.
\end{abstract}

\section{Contents}

1. Introduction

2. Energy estimate

3. $L^{6}$ estimate of $\mathbf{P}_{\mathfrak{u}} f$

4. Balance laws

5. Estimate of $\left\|\mathbf{P}_{\mathfrak{u}} f\right\|_{3}$

1

5.1. Splitting of $\mathbf{P}_{\mathfrak{u}} f$

5.2. Estimate of $\mathbf{S}_{1} f$

5.3. Estimate of $\mathbf{S}_{2} f$

5.4. Estimate of $\mathbf{S}_{3} f$

5.5. Estimate of $\mathbf{S}_{4} f$

5.6. Estimate of $\mathbf{S}_{5} f$

5.7. Proof of Theorem 1.5

Pruction of the positive solution to the non linear problem

6.1. Positivity scheme 34

6.2. Iteration 39

Appendix A. Bounds on the velocity field 42

References

\section{INTRODUCTION}

Let $\Omega$ be a smooth bounded open subset of $\mathbb{R}^{3}$ and $\bar{\Omega}$ its closure. A gas moves in $\Omega^{c}=\mathbb{R}^{3} \backslash \bar{\Omega}$ with prescribed velocity $\mathfrak{u}$ at infinity and vanishing velocity on $\partial \Omega$, evolving according to the incompressible Navier-Stokes equations. The steady boundary value problem for this system is classical in Fluid Mechanics and a huge literature has been devoted to it [2, 12, 19, 20, 22, 27. (see also [13] and references quoted therein). One of the main difficulties of this problem is related to the presence of the "wake" [29] and the corresponding slow decay to $\mathfrak{u}$ of the velocity field at infinity.

In the case of a rarefied gas, an alternative description is possible in terms of the Boltzmann equation and suitable boundary conditions. In this paper we study the link between these two descriptions in the small Knudsen numbers and low Mach numbers regime.

(R. E.) International Research Center M\&MOCS, Univ. dell'Aquila, Cisterna di Latina, (LT) 04012 Italy.

(Y. G.) Division of Applied Mathematics, Brown University, Providence, RI 02812, U.S.A..

(R. M.) Dipartimento di Fisica and Unità INFN, Università di Roma Tor Vergata, 00133 Roma, Italy. 
It is well known that in this regime the time dependent Boltzmann equation behaves as the incompressible Navier-Stokes equation, 3, 4, 8, 15, 16, 17, 23, 25, 28. Much less is know for the corresponding steady Boltzmann problem, where the natural $L^{1}$ and entropy estimates are not available, and only the entropy production can be exploited.

Ukai and Asano [30, 31, see also [32, studied the Boltzmann equation in the exterior domain with fixed Knudsen number. They considered a rarefied gas outside a piecewise smooth convex domain of $\mathbb{R}^{3}$, with suitable boundary conditions and a prescribed Maxwellian behavior at infinity. The Maxwellian at infinity was centered at a small velocity field. For this problem Ukai and Asano were able to prove existence of the steady solution and its dynamical stability.

Our main result is the construction of the steady solution to the Boltzmann equation in the exterior domain and the estimate of its closeness to the steady incompressible Navier Stokes equation when Knudsen and Mach numbers are small. Recently in [10] we have constructed the solution to the Boltzmann equation for small Knudsen and Mach numbers in a smooth bounded domain, under the action of a suitably small external force and small variations of the boundary temperature. The exterior problem is even more difficult, due to the need of good decay properties for large $x$.

Before describing the difficulties to achieve our program, let us state more precisely the problem and the result.

We assume that $\Omega \subset \mathbb{R}^{3}$ is a $C^{2}$ bounded domain, not necessarily convex. Let $x \in \Omega^{c}=\mathbb{R}^{3} \backslash \bar{\Omega}$ and $v \in \mathbb{R}^{3}$. Let $F(x, v) \geq 0$ be the (unnormalized) distribution function of a rarefied gas in $\Omega^{c}$ with position $x$ and velocity $v$, satisfying the steady Boltzmann equation

$$
v \cdot \nabla F=\frac{1}{\varepsilon} Q(F, F), \quad \text { in } \Omega^{c}
$$

where $\nabla \equiv \nabla_{x}$ and

$$
\begin{aligned}
Q(f, g)(v) & =Q^{+}(f, g)-Q^{-}(f, g), \\
Q^{+}(f, g)(v) & =\int_{\mathbb{R}^{3}} \mathrm{~d} v_{*} \int_{\left\{\omega \in \mathbb{R}^{3}:|\omega|=1\right\}} \mathrm{d} \omega B\left(\omega, v-v_{*}\right) f\left(v^{\prime}\right) g\left(v_{*}^{\prime}\right), \\
Q^{-}(f, g)(v) & =f(v) \int_{\mathbb{R}^{3}} v_{*} \int_{\left\{\omega \in \mathbb{R}^{3}:|\omega|=1\right\}} \mathrm{d} \omega B\left(\omega, v-v_{*}\right) g\left(v_{*}\right) .
\end{aligned}
$$

Here $v^{\prime}$ and $v_{*}^{\prime}$ are the incoming velocities in the elastic collision, defined by

$$
v^{\prime}=v-\omega\left(v-v_{*}\right) \cdot \omega, \quad v_{*}^{\prime}=v_{*}+\omega\left(v-v_{*}\right) \cdot \omega,
$$

and $B(\omega, V)$ is the cross section for hard potentials with Grad's angular cutoff, so that $\int_{\{|\omega|=1\}} \mathrm{d} \omega B(V, \omega) \lesssim|V|^{\theta}$ for $0 \leq \theta \leq 1$ depending on the interaction potential. In particular, $B(\omega, V)=|\omega \cdot V|$ for hard spheres and $\theta=1$.

We assume diffuse reflection boundary condition: Let $\gamma=\partial \Omega \times \mathbb{R}^{3}=\gamma_{+} \cup \gamma_{-} \cup \gamma_{0}$, with

$$
\gamma_{ \pm}=\left\{(x, v) \in \partial \Omega \times \mathbb{R}^{3}: n(x) \cdot v \gtrless 0\right\}, \quad \gamma_{0}=\left\{(x, v) \in \partial \Omega \times \mathbb{R}^{3}: n(x) \cdot v=0\right\},
$$

$n(x)$ denoting the normal at $x$ to $\partial \Omega$ pointing inside $\Omega$. Let

$$
M_{\rho, u, T}:=\frac{\rho}{(2 \pi T)^{\frac{3}{2}}} \exp \left[-\frac{|v-u|^{2}}{2 T}\right],
$$

be the local Maxwellian with density $\rho$, mean velocity $u$, and temperature $T$ and

$$
\mu=M_{1,0,1}=\frac{1}{(2 \pi)^{\frac{3}{2}}} \exp \left[-\frac{|v|^{2}}{2}\right] .
$$

On the boundary $F$ satisfies the diffuse reflection condition defined as

$$
F(x, v)=\mathcal{P}_{\gamma}^{w}(F)(x, v) \quad \text { on } \gamma_{-},
$$

where

$$
\mathcal{P}_{\gamma}^{w}(F)(x, v):=M^{w}(x, v) \int_{\{n(x) \cdot w>0\}} \mathrm{d} w F(x, w)\{n(x) \cdot w\}
$$


with the wall Maxwellian defined as

$$
M^{w}=\sqrt{2 \pi} \mu=\frac{1}{2 \pi} \exp \left[-\frac{|v|^{2}}{2}\right], \quad \int_{\{v \cdot n \gtrless 0\}} \mathrm{d} v M^{w}(v)|n \cdot v|=1 .
$$

We also specify the condition at infinity. Since we study the problem in the small Mach number regime, we assume that the velocity at infinity is of order $\varepsilon$. In other words, fixed a constant vector $\mathfrak{u}$, denoting

$$
v_{\mathfrak{u}}:=v-\varepsilon \mathfrak{u}, \quad \mu_{\mathfrak{u}}(v):=\mu\left(v_{\mathfrak{u}}\right)=M_{1, \varepsilon \mathfrak{u}, 1}(v),
$$

we assume in a suitable sense

$$
\lim _{|x| \rightarrow \infty} F(x, v)=\mu_{\mathfrak{u}}(v) .
$$

Note that we have prescribed the same uniform temperature on $\partial \Omega$ and at infinity for sake of simplicity, but we believe that a temperature difference of order $\varepsilon$ could be included. We do not discuss this. The case of sufficiently small difference of temperature for fixed $\varepsilon$ has been discussed in [33].

Let the couple velocity field and pressure, $(U, p)$, be solution to the Stationary Incompressible Navier-Stokes equation (SINS) in $\Omega^{c}$ :

$$
U \cdot \nabla U+\nabla P=\mathfrak{v} \Delta U, \quad \nabla \cdot U=0, \quad U=0 \text { on } \partial \Omega, \quad U \rightarrow \mathfrak{u}, \text { as }|x| \rightarrow \infty
$$

where $\mathfrak{v}>0$ is the viscosity coefficient. It is convenient to represent $U=u+\mathfrak{u}$, with $(u, P)$ solving

$$
(\mathfrak{u}+u) \cdot \nabla u+\nabla P=\mathfrak{v} \Delta u, \quad \nabla \cdot u=0, \quad u=-\mathfrak{u} \text { on } \partial \Omega, \quad u \rightarrow 0, \text { as }|x| \rightarrow \infty .
$$

Solutions to this equation do exist in $L^{p}$, for any $p>2$ and uniqueness is ensured for $|\mathfrak{u}|$ small (see e.g. [13, Thm. X.6.4).

Our aim is to show that $F \approx M_{1, \varepsilon(u+\mathfrak{u}), 1}$ as $\varepsilon \rightarrow 0$. More precisely, since $M_{1, \varepsilon(u+\mathfrak{u}), 1}=$ $\mu_{\mathfrak{u}}+\varepsilon f_{1} \sqrt{\mu}_{\mathfrak{u}}+O\left(\varepsilon^{2}\right)$, where

$$
f_{1}=\sqrt{\mu_{\mathfrak{u}}} u \cdot v_{\mathfrak{u}}
$$

we need to show that $\varepsilon^{-1}\left(F-\mu_{\mathfrak{u}}\right) \approx f_{1} \sqrt{\mu}_{\mathfrak{u}}$ as $\varepsilon \rightarrow 0$ is in $L^{p}$ for any $p>2$, with the same decay of $u$. Therefore, we set $\tilde{R}=\varepsilon^{-\frac{1}{2}} \mu_{\mathfrak{u}}^{-\frac{1}{2}}\left[F-\mu_{\mathfrak{u}}-\varepsilon f_{1} \sqrt{\mu_{\mathfrak{u}}}\right]$ and write the equation for $\tilde{R}$. Let $L_{\mathfrak{u}}$ be the usual linearized Boltzmann operator defined as

$$
L_{\mathfrak{u}} f=-\mu_{\mathfrak{u}}^{-\frac{1}{2}}\left[Q\left(\mu_{\mathfrak{u}}, \mu_{\mathfrak{u}}^{\frac{1}{2}} f\right)+Q\left(\mu_{\mathfrak{u}}^{\frac{1}{2}} f, \mu_{\mathfrak{u}}\right)\right]:=\nu f-K f,
$$

where: $\nu(v)=\int_{\mathbb{R}^{3} \times\{|\omega|=1\}} d v_{*} d \omega B\left(v-v_{*}, \omega\right) \mu\left(v_{*}\right)$ is such that $0 \leq \nu_{0}|v|^{\theta} \leq \nu(v) \leq \nu_{1}|v|^{\theta} ; K$ is a compact operator on $L^{2}\left(\mathbb{R}_{v}^{3}\right) . L_{\mathfrak{u}}$ is an operator on $L^{2}\left(\mathbb{R}_{v}^{3}\right)$ whose null space is

$$
\text { Null } L_{\mathfrak{u}}=\operatorname{span}\left\{1, v_{\mathfrak{u}},\left|v_{\mathfrak{u}}\right|^{2}\right\} \sqrt{\mu_{\mathfrak{u}}},
$$

Let $\mathbf{P}_{\mathfrak{u}}$ be the orthogonal projector on Null $L_{\mathfrak{u}}$. In particular, $L_{\mathfrak{u}} f_{1}=0$. Thus we have

$$
v \cdot \nabla \tilde{R}+\varepsilon^{-1} L_{\mathfrak{u}} \tilde{R}=\left[\Gamma_{\mathfrak{u}}\left(f_{1}, \tilde{R}\right)+\Gamma_{\mathfrak{u}}\left(\tilde{R}, f_{1}\right)\right]+\varepsilon^{\frac{1}{2}} \Gamma_{\mathfrak{u}}(\tilde{R}, \tilde{R})+\varepsilon^{-\frac{1}{2}}\left[\Gamma_{\mathfrak{u}}\left(f_{1}, f_{1}\right)-v \cdot \nabla f_{1}\right],
$$

where

$$
\begin{aligned}
\Gamma_{\mathfrak{u}}(f, g) & =\Gamma_{\mathfrak{u}}^{+}(f, g)-\Gamma_{\mathfrak{u}}^{-}(f, g), \\
\Gamma_{\mathfrak{u}}^{ \pm}(f, g) & =\mu_{\mathfrak{u}}^{-\frac{1}{2}} Q^{ \pm}\left(\mu_{\mathfrak{u}}^{\frac{1}{2}} f, \mu_{\mathfrak{u}}^{\frac{1}{2}} g\right), \\
\tilde{\Gamma}_{\mathfrak{u}}(f, g) & =\frac{1}{2}\left[\Gamma_{\mathfrak{u}}(f, g)+\Gamma_{\mathfrak{u}}(g, f)\right] .
\end{aligned}
$$

To remove the divergent term in (1.18), we note that, since $\nabla \cdot u=0$, then

$$
\mathbf{P}_{\mathfrak{u}}\left(v_{\mathfrak{u}} \cdot \nabla f_{1}\right)=0,
$$

and

$$
f_{2}=L_{\mathfrak{u}}^{-1}\left[-\left(\mathbf{I}-\mathbf{P}_{\mathfrak{u}}\right)\left[v_{\mathfrak{u}} \cdot \nabla f_{1}\right]+\Gamma_{\mathfrak{u}}\left(f_{1}, f_{1}\right)\right]
$$

is well defined and is in $L^{p}$ for any $p>\frac{4}{3}$, because so is $\nabla u$ (see e.g. [13, Thm. X.6.4). Since $u$ solves the SINS equation, then it is easy to check that

$$
\mathbf{P}_{\mathfrak{u}}\left[v_{\mathfrak{u}} \cdot \nabla f_{2}+\mathfrak{u} \cdot \nabla f_{1}\right]=0 .
$$


Therefore, by setting $R=\tilde{R}-\varepsilon^{\frac{1}{2}} f_{2}$, which means $F=\mu_{\mathfrak{u}}+\varepsilon\left(f_{1}+\varepsilon f_{2}+\varepsilon^{\frac{1}{2}} R\right) \sqrt{\mu_{\mathfrak{u}}}$, we see that $F$ is a stationary solution to (1.1) if and only if $R$ solves the equation:

$$
v \cdot \nabla R+\varepsilon^{-1} L_{\mathfrak{u}} R=L_{\mathfrak{u}}^{(1)} R+\varepsilon^{\frac{1}{2}} \Gamma_{\mathfrak{u}}(R, R)+\varepsilon^{\frac{1}{2}} A_{\mathfrak{u}},
$$

where

$$
\begin{gathered}
L_{\mathfrak{u}}^{(1)} R=2 \tilde{\Gamma}_{\mathfrak{u}}\left(f_{1}+\varepsilon f_{2}, R\right) \\
A_{\mathfrak{u}}=-\left(\mathbf{I}-\mathbf{P}_{\mathfrak{u}}\right)\left[v \cdot \nabla f_{2}\right]+2 \tilde{\Gamma}_{\mathfrak{u}}\left(f_{1}, f_{2}\right)+\varepsilon \Gamma_{\mathfrak{u}}\left(f_{2}, f_{2}\right)-\varepsilon \mathfrak{u} \cdot \nabla f_{2} .
\end{gathered}
$$

Since $u \rightarrow 0$ at $\infty$, then $f_{1}$ and $f_{2}$ also go to 0 at $\infty$. Thus we have to impose

$$
\lim _{|x| \rightarrow \infty} R=0 .
$$

For $f \in L^{1}\left(\gamma_{ \pm}\right)$we define

$$
P_{\gamma}^{\mathfrak{u}} f=\mu_{\mathfrak{u}}^{-\frac{1}{2}} \mathcal{P}_{\gamma}^{w}\left[\mu_{\mathfrak{u}}^{\frac{1}{2}} f\right]=\sqrt{2 \pi} \frac{\mu}{\sqrt{\mu}_{\mathfrak{u}}} z_{\gamma_{+}}(f), \quad z_{\gamma_{ \pm}}(f)(x)=\int_{\{v \cdot n(x) \gtrless 0\}} \mathrm{d} v \sqrt{\mu}_{\mathfrak{u}}(v)|v \cdot n(x)| f(x, v),
$$

$z_{\gamma_{ \pm}}(f)(x)$ being the outgoing/incoming mass flux at $x \in \partial \Omega$. We will omit the index \pm when there is no ambiguity.

The boundary condition for $R$ is:

$$
R=P_{\gamma}^{\mathfrak{u}} R+\varepsilon^{\frac{1}{2}} r
$$

where

$$
r=P_{\gamma}^{\mathfrak{u}}\left[f_{2}-\phi_{\varepsilon}\right]-\left(f_{2}-\phi_{\varepsilon}\right), \quad \text { on } \gamma_{-},
$$

with $\phi_{\varepsilon}$ defined as

$$
\phi_{\varepsilon}=\varepsilon^{-2} \mu_{\mathfrak{u}}^{-\frac{1}{2}}\left[M_{1, \varepsilon(\mathfrak{u}+u), 1}-\mu_{\mathfrak{u}}-\varepsilon \sqrt{\mu_{\mathfrak{u}}} f_{1}\right],
$$

such that

$$
\left|\phi_{\varepsilon}\right| \leq C_{\beta}\left(|u|^{2}+|\mathfrak{u}|^{2}\right) \exp \left[-\beta|v|^{2}\right] \quad \text { for any } \beta<\frac{1}{4} .
$$

Indeed, for $x \in \partial \Omega$, where $u(x)=-\mathfrak{u}$, we have $\mu=M_{1, \varepsilon(\mathfrak{u}+u), 1}$ and hence

$$
\mu=\left.M_{1, \varepsilon(\mathfrak{u}+u), 1}\right|_{\partial \Omega}=\mu_{\mathfrak{u}}+\left.\varepsilon \sqrt{\mu_{\mathfrak{u}}} f_{1}\right|_{\partial \Omega}+\left.\varepsilon^{2} \sqrt{\mu_{\mathfrak{u}}} \phi_{\varepsilon}\right|_{\partial \Omega} .
$$

and, in consequence of $\mu=\mathcal{P}_{\gamma}^{w} \mu$, on $\gamma_{-}$we have

$$
\mu_{\mathfrak{u}}+\varepsilon f_{1} \mu_{\mathfrak{u}}^{\frac{1}{2}}+\varepsilon^{2} \phi_{\varepsilon} \mu_{\mathfrak{u}}^{\frac{1}{2}}=\mathcal{P}_{\gamma}^{w}\left[\mu_{\mathfrak{u}}+\varepsilon f_{1} \mu_{\mathfrak{u}}^{\frac{1}{2}}+\varepsilon^{2} \phi_{\varepsilon} \mu_{\mathfrak{u}}^{\frac{1}{2}}\right] .
$$

On the other hand the boundary condition (1.8) for $F$ gives on $\gamma_{-}$,

$$
\mu_{\mathfrak{u}}+\varepsilon f_{1} \mu_{\mathfrak{u}}^{\frac{1}{2}}+\varepsilon^{2} f_{2} \mu_{\mathfrak{u}}^{\frac{1}{2}}+\varepsilon^{\frac{3}{2}} R \mu_{\mathfrak{u}}^{\frac{1}{2}}=\mathcal{P}_{\gamma}^{w}\left[\mu_{\mathfrak{u}}+\varepsilon f_{1} \mu_{\mathfrak{u}}^{\frac{1}{2}}+\varepsilon^{2} f_{2} \mu_{\mathfrak{u}}^{\frac{1}{2}}+\varepsilon^{\frac{3}{2}} R \mu_{\mathfrak{u}}^{\frac{1}{2}}\right] .
$$

Therefore, subtracting the last two equations

$$
\varepsilon^{2} f_{2} \mu_{\mathfrak{u}}^{\frac{1}{2}}+\varepsilon^{\frac{3}{2}} R \mu_{\mathfrak{u}}^{\frac{1}{2}}-\varepsilon^{2} \phi_{\varepsilon} \mu_{\mathfrak{u}}^{\frac{1}{2}}=\mathcal{P}_{\gamma}^{w}\left[\varepsilon^{2} f_{2} \mu_{\mathfrak{u}}^{\frac{1}{2}}+\varepsilon^{\frac{3}{2}} R \mu_{\mathfrak{u}}^{\frac{1}{2}}-\varepsilon^{2} \phi_{\varepsilon} \mu_{\mathfrak{u}}^{\frac{1}{2}}\right],
$$

which implies (1.28).

Note that, from the definition of $A_{\mathfrak{u}}$, it follows that

$$
\mathbf{P}_{\mathfrak{u}} A_{\mathfrak{u}}=0 .
$$

Moreover, it can be checked that

$$
z_{\gamma_{-}}(r)=\int_{\{v \cdot n<0\}} \mathrm{d} v r \sqrt{\mu_{\mathfrak{u}}} n \cdot v=0 .
$$

From the definition of $r$ it follows that

$$
|r|_{2,-}+|r|_{\infty} \lesssim|\mathfrak{u}|
$$


Notation. Depending on the context, we denote $\|f\|_{p}=\|f\|_{L^{p}\left(\Omega_{x}^{c} \times \mathbb{R}_{v}^{3}\right)}$ or $\|f\|_{p}=\|f\|_{L^{p}\left(\Omega_{x}^{c}\right)}$ or $\|f\|_{p}=\|f\|_{L^{p}(\partial \Omega)}$ for $1 \leq p \leq \infty$. $\|f\|_{\nu}=\left\|f \nu^{\frac{1}{2}}\right\|_{2}$. We set $|f|_{p, \pm}=\left(\int_{\gamma_{ \pm}} \mathrm{d} \gamma|f(x, v)|^{p}\right)^{\frac{1}{p}}$, with

$$
\int_{\gamma_{ \pm}} f \mathrm{~d} \gamma=\int_{\partial \Omega} d S(x) \int_{\{v \cdot n(x) \gtrless 0\}} \mathrm{d} v|v \cdot n(x)| f(x, v) .
$$

Finally, we define

$$
\left[[f]_{\beta, \beta^{\prime}}=\varepsilon^{-1}\left\|\left(\mathbf{I}-\mathbf{P}_{\mathfrak{u}}\right) f\right\|_{\nu}+\varepsilon^{-\frac{1}{2}}\left|\left(1-P_{\gamma}^{\mathfrak{u}}\right) f\right|_{2,+}+\left\|\mathbf{P}_{\mathfrak{u}} f\right\|_{6}+\varepsilon^{\frac{1}{2}}\left\|\mathbf{P}_{\mathfrak{u}} f\right\|_{3}+\varepsilon^{\frac{1}{2}}\|w f\|_{\infty}\right.
$$

with the weight function $w(v)=\langle v\rangle^{\beta^{\prime}} \exp \left[\beta|v|^{2}\right]$, where $\langle v\rangle=\left(1+|v|^{2}\right)^{\frac{1}{2}}$.

The main result is

Theorem 1.1. Let $\Omega$ be a $C^{2}$ bounded open set of $\mathbb{R}^{3}$ and $\Omega^{c}=\mathbb{R}^{3} \backslash \bar{\Omega}$. Fix $\mathfrak{u} \in \mathbb{R}^{3}$ such that $0<|\mathfrak{u}| \ll 1$. For any $0<\varepsilon \ll 1$ consider the steady boundary value problem

$$
\left\{\begin{array}{l}
v \cdot \nabla F=\frac{1}{\varepsilon} Q(F, F), \quad \text { in } \Omega^{c} \\
F(x, v)=M^{w} \int_{\{v \cdot n>0\}} F v \cdot n \mathrm{~d} v \text { on } \gamma_{-}, \\
\lim _{|x| \rightarrow \infty} F(x, v)=\mu_{\mathfrak{u}}(v) .
\end{array}\right.
$$

Then

- the problem (1.39) has a positive solution which can be represented as

$$
F=\mu_{\mathfrak{u}}+\sqrt{\mu}_{\mathfrak{u}}\left[\varepsilon f_{1}+\varepsilon^{2} f_{2}+\varepsilon^{\frac{3}{2}} R\right],
$$

with $f_{1}$ and $f_{2}$ given by (1.15) and (1.21), $u$ solving (1.14), and $R$ solving (1.23), (1.28).

- $R$ satisfies the bound

for $\beta^{\prime} \geq 0$ and $0<\beta \ll \frac{1}{4}$.

$$
[[R]]_{\beta, \beta^{\prime}} \lesssim|\mathfrak{u}|,
$$

- $R$ is unique in the ball $\left\{f: \llbracket[f]_{0, \beta^{\prime}} \lesssim|\mathfrak{u}|\right\}$.

Remark 1.2. Note that while the $L^{2}$ norm of $\left(\mathbf{I}-\mathbf{P}_{\mathfrak{u}}\right) R$ is bounded and actually small as $\varepsilon \rightarrow 0$, $\mathbf{P}_{\mathfrak{u}} R$ is bounded uniformly in $\varepsilon$ only in $L^{6}$, while the $L^{3}$ and $L^{\infty}$ bounds of $\mathbf{P}_{\mathfrak{u}} R$ are divergent with $\varepsilon^{-\frac{1}{2}}$. It turns out that that the $L^{p}$ norm of $\mathbf{P}_{\mathfrak{u}} R$ is bounded for $p>2$, but the bound is not uniform in $\varepsilon$ for $2<p<6$. This is the counterpart of the slow decay of the velocity field $u$ at infinity, which is well known in Fluid Dynamics, where it is proved that the $L^{2}$ norm of $u$ is unbounded. We do not know if a similar statement is true for $R$, but it is certainly true for $f_{1}$ which is linear in $u$ and hence for $\varepsilon^{-1}\left(F-\mu_{\mathfrak{u}}\right)$.

Remark 1.3. We also note that combining the estimates implied by (1.41), it follows that $\|R\|_{6}$ is bounded uniformly in $\varepsilon$. In fact, we have $\left\|\mathbf{P}_{\mathfrak{u}} R\right\|_{6} \leq[[R] \lesssim|\mathfrak{u}|$ and

$$
\left\|\left(\mathbf{I}-\mathbf{P}_{\mathfrak{u}}\right) R\right\|_{6} \leq\left\|\left(\mathbf{I}-\mathbf{P}_{\mathfrak{u}}\right) R\right\|_{2}^{\frac{1}{3}}\|R\|_{\infty}^{\frac{2}{3}} \leq(\varepsilon[[R]])^{\frac{1}{3}}\left(\varepsilon^{-\frac{1}{2}}[[R]]\right)^{\frac{2}{3}} \leq[[R]\rfloor|\mathfrak{u}| .
$$

Since $f_{1}$ and $f_{2}$ are also bounded in $L^{6}$, uniformly in $\varepsilon$, we conclude that $\varepsilon^{-1}\left(F-\mu_{\mathfrak{u}}\right)$ is bounded in $L^{6}$ uniformly in $\varepsilon$. The condition at infinity for $F$ is verified in this sense.

Remark 1.4. The uniqueness is proved in the ball $\left\{f:\left[[f]_{0, \beta^{\prime}} \lesssim|\mathfrak{u}|\right\}\right.$. No exponential decay in $v$ is required for uniqueness.

In Sections 25 we shall consider the following linear problem:

$$
\begin{cases}v \cdot \nabla f+\varepsilon^{-1} L_{\mathfrak{u}} f=g, & (x, v) \in \Omega^{c} \\ f=P_{\gamma}^{\mathfrak{u}} f+\varepsilon^{\frac{1}{2}} r, & (x, v) \in \gamma_{-}, \\ \lim _{|x| \rightarrow \infty} f=0 . & \end{cases}
$$


By (1.34) and (1.35), $\mathbf{P}_{\mathfrak{u}} g=0$ and $z_{\gamma_{-}}(r)=0$ in the linearization of the problem (1.23), (1.28). However, to prove the positivity of the solution to (1.1) we are going to construct, we have to suitably modify the equation (1.1) and in the resulting linear problem to be studied (1.34) and (1.35) is no more exact but $\mathbf{P}_{\mathfrak{u}} g$ and $z_{\gamma}(r)$ is small for $\varepsilon$ small. Therefore in the next sections we shall drop the condition (1.34) and (1.35).

We shall prove the following

Theorem 1.5. Fixed $\mathfrak{u}$ with $0<|\mathfrak{u}| \ll 1$, if $\varepsilon \ll 1$, the solution to the linear problem (1.42) satisfies the inequality

$$
[[f]]_{\beta, \beta^{\prime}}^{2} \lesssim \mathscr{M}(g, r)
$$

where

$$
\begin{aligned}
& \mathscr{M}(g, r)=\left\|\nu^{-\frac{1}{2}}\left(\mathbf{I}-\mathbf{P}_{\mathfrak{u}}\right) g\right\|_{2}^{2}+\varepsilon\left\|\nu^{-\frac{1}{2}} g\right\|_{\frac{3}{2}}^{2}+\varepsilon^{3}\left\|\langle v\rangle^{-1} w g\right\|_{\infty}^{2}+\varepsilon|w r|_{\infty,-}^{2}+|r|_{2,-}^{2} \\
& +\left\|\mathbf{P}_{\mathfrak{u}} g\right\|_{2}^{2}+\varepsilon^{-2}|\mathfrak{u}|^{-2}\left\|\mathbf{P}_{\mathfrak{u}} g\right\|_{\frac{6}{5}}^{2}+\varepsilon^{-1}|\mathfrak{u}|^{-2+2 \varrho}\left\|\mathbf{P}_{\mathfrak{u}} g\right\|_{\frac{6}{5}}^{2}+\left(|\mathfrak{u}|^{-2+2 \varrho}+|\mathfrak{u}|^{-1} \varepsilon^{-1}\right)\left\|z_{\gamma}(r)\right\|_{2}^{2},
\end{aligned}
$$

for $\beta^{\prime} \geq 0$ and $0 \leq \beta \ll \frac{1}{4}$ and $\rho>0$.

Remark 1.6. We note that the second line of (1.44) vanishes when the hydrodynamic part of $g$ and the net mass flux of $r$ vanishes. This is the case for the problem (1.23), (1.28). In the modified problem introduced for the proof of positivity it does not vanish, but its contribution turns out to be small.

Before going into a short sketch of the arguments we use, it is worth to comment the choice of the power of $\varepsilon$ in front of $R, \alpha=\frac{3}{2}$. Clearly, to deal with the non linear term is easier when this power is large. However we are limited by the fact that $f_{2}$ does not satisfy the boundary conditions and a power $\alpha>2$ would require the introduction of a boundary layer correction with serious regularity issues due to the general geometry (see 34 for the analysis of such problems). On the other hand $\alpha \leq \frac{3}{2}$ is required to avoid a divergent contribution from the boundary terms in the energy inequality. It turns out that the value $\alpha=\frac{3}{2}$ is exactly what we need to bound the non linear term thanks to the uniform estimate we are able to obtain for $\varepsilon^{\frac{1}{2}}\left\|\mathbf{P}_{\mathfrak{u}} R\right\|_{3}$.

Our analysis relies crucially on energy inequality to control entropy production. It gives important information: the microscopic part of the solution $\left(\mathbf{I}-\mathbf{P}_{\mathfrak{u}}\right) R$ is of order $\varepsilon$ in $L^{2}$ and moreover $\left|\left(1-P_{\gamma}^{\mathfrak{u}}\right) R\right|_{2,+} \sim \sqrt{\varepsilon}$.

Our main technical achievement is establishing the linear estimate (1.43), $[[f]]_{\beta, \beta^{\prime}}^{2} \lesssim \mathscr{M}(g, r)$. The starting point is a new $L^{6}$ estimate for $\mathbf{P}_{\mathfrak{u}} f$ in Section 3 , which extends the one in the recent paper [10, while the $L^{\infty}$ estimate follows directly from [10. The key observation is that the $L^{6}$ estimate for the macroscopic part of $R, \mathbf{P}_{\mathfrak{u}} R$, is valid in the unbounded exterior region, thanks to scaling invariance in the homogeneous Sobolev space $\dot{H}^{1}$. The proof, which requires a weak formulation and a careful choice of the test functions, is also based on delicate estimates of the boundary terms.

However, to deal with the nonlinear part $\Gamma_{\mathfrak{u}}(R, R)$, the $L^{6}$ estimate is not sufficient, some control of the $L^{3}$ estimate is required. Unlike in the bounded domains, the $L^{6}$ bound alone cannot imply $L^{3}$ bound, for $|x| \rightarrow \infty$. In fact, the $L^{3}$ bound requires faster decay as $|x| \rightarrow \infty$, which is a much stronger estimate than $L^{6}$ estimate. This gain of lower integrability near infinity can be viewed as opposite to the velocity averaging ideas which lead to higher integrability gain for bounded $|x|$. In fact, starting from the bound for $L^{6}$ norm, we need to show bounds on lower $p$ 's norms. By working on the balance laws we can prove a uniform in $\varepsilon$ bound for $\varepsilon^{\frac{1}{2}}\left\|\mathbf{P}_{\mathfrak{u}} f\right\|_{3}$ for $|x| \gg 1$, which is sufficient to close our estimate (Sections 6).

To this purpose, inspired by Maslova, 24], in Section 4, after multiplying the equation by a smooth spatial cutoff function $\zeta$ vanishing at $\partial \Omega$, we rewrite the macroscopic projection of the linear Boltzmann equation for $f^{\zeta}=\zeta f$ as a (non closed) system for $\mathbf{P}_{\mathfrak{u}} f^{\zeta}$ in the whole space (see Eq.(4.32), (4.33), (4.34) ) (In [24] a similar system was introduced to solve the steady Boltzmann equation with $\varepsilon=1$, with in-flow boundary condition and asymptotic Maxwellian with prescribed 
mean velocity at infinity):

$$
\begin{aligned}
\nabla_{x} \cdot b^{\zeta}+\varepsilon \mathfrak{u} \cdot \nabla_{x} a^{\zeta} & =s_{0}, \\
\nabla_{x}\left(a^{\zeta}+c^{\zeta}\right)-\varepsilon \mathfrak{v} \Delta b^{\zeta}+\varepsilon \mathfrak{u} \cdot \nabla_{x} b^{\zeta} & =\underline{s}, \\
\nabla_{x} \cdot b^{\zeta}-\varepsilon \kappa \Delta c^{\zeta}+\frac{3}{2} \varepsilon \mathfrak{U} \cdot \nabla_{x} c^{\zeta} & =s_{4},
\end{aligned}
$$

where $\mathbf{P}_{\mathfrak{u}} f^{\zeta}=\left[a^{\zeta}+b^{\zeta} \cdot v_{\mathfrak{u}}+\frac{1}{2} c^{\zeta}\left(\left|v_{\mathfrak{u}}\right|^{2}-3\right)\right] \sqrt{\mu}_{\mathfrak{u}}$ and the sources $s_{0}, \underline{s}, s_{4}$ depend on $f$ and on $\zeta$. For $|\mathfrak{u}| \ll 1$ we study the above system via Fourier analysis, by means of a decomposition of $\mathbf{P}_{\mathfrak{u}} f^{\zeta}$ into high-frequency and low-frequency parts. Of course, in the large $|x|$ regime the lowfrequency part is the difficult one and its treatment requires a further decomposition in different contributions, the most delicate being the one for the total mass, momentum and energy fluxes at the boundary, needed in Lemma 5.5 which are obtained thanks to the condition $\mathfrak{u} \neq 0$, an ingredient also entering crucially in the Fluid Dynamic treatment of the problem (see e.g. [13]). We establish in Section [5 very precise $L^{p}$ estimates $p>2$ for the different parts of $\mathbf{P}_{\mathfrak{u}} f$, because $\mathfrak{u} \neq 0$ ensures more integrability than in the corresponding Stokes system. It is worth to stress that such arguments, however accurate they are, only produce an estimate of $\left\|\mathbf{P}_{\mathfrak{u}} f\right\|_{p} \sim \varepsilon^{-1}$, which would not be good enough for our purposes, we need at most $\left\|\mathbf{P}_{\mathfrak{u}} f\right\|_{3} \sim \varepsilon^{-\frac{1}{2}}$ to deal with the non linearity because of the limitation explained before. It is only thanks to the essential uniform in $\varepsilon$ estimate of $\left\|\mathbf{P}_{\mathfrak{u}} f\right\|_{6} \sim 1$, that, via a careful estimate of the mass momentum and energy fluxes at the boundary in Subsection 5.3 and interpolation, we can obtain a bound $\sqrt{\varepsilon}\left\|\mathbf{P}_{\mathfrak{u}} f\right\|_{3} \sim 1$, uniform in $\varepsilon$.

It is well-known that it is challenging to prove positivity for steady Boltzmann solutions. We succeed in this by suitably adapting and extending the positivity-preserving scheme of Arkeryd and Nouri [1. When dealing with the diffuse reflection boundary condition for this new scheme we encounter an extra difficulty with a new term determining a potential violation of the vanishing net mass flux condition at the boundary, that is controlled via accurate estimates in the large velocity set and the Ukai trace theorem [30].

Finally we prove our main theorem in Section 6 via iteration, based on the linear estimate (1.43). A crucial information we need to close the iteration is the smallness of the velocity field when $|\mathfrak{u}|$ is small. This estimate is proven in the Appendix $\mathrm{A}$.

\section{ENERGY ESTIMATE}

We shall use in many points the following two lemmas whose proof is standard and can be found for example in $[9]$ :

Lemma 2.1. Assume that $f(x, v), h(x, v) \in L^{p}\left(\Omega^{c} \times \mathbb{R}^{3}\right), p \geq 2$ and $v \cdot \nabla_{x} f, v \cdot \nabla_{x} h \in L^{\frac{p}{p-1}}\left(\Omega^{c} \times\right.$ $\left.\mathbb{R}^{3}\right)$ and $\left.f\right|_{\gamma},\left.h\right|_{\gamma} \in L^{2}\left(\partial \Omega \times \mathbb{R}^{3}\right)$. Then

$$
\iint_{\Omega^{c} \times \mathbb{R}^{3}} \mathrm{~d} x \mathrm{~d} v\left[\left(v \cdot \nabla_{x} h\right) f+\left(v \cdot \nabla_{x} f\right) h\right]=\int_{\gamma_{+}} \mathrm{d} \gamma f h-\int_{\gamma_{-}} \mathrm{d} \gamma f h .
$$

Lemma 2.2. Assume $\Omega_{1}$ is an open bounded subset of $\mathbb{R}^{3}$ with $\partial\left(\Omega_{1} \backslash \bar{\Omega}\right)$ in $C^{2}$, such that $\{x \in$ $\left.\Omega^{c} \mid d(x, \Omega) \leq 1\right\} \subset \Omega_{1}$. We define

$$
\gamma_{ \pm}^{\delta}:=\left\{(x, v) \in \gamma_{ \pm}:|n(x) \cdot v|>\delta, \quad \delta \leq|v| \leq \frac{1}{\delta}\right\} .
$$

Then

$$
\left|f \mathbf{1}_{\gamma_{ \pm}^{\delta}}\right|_{1} \lesssim \delta, \Omega_{1}\|f\|_{L^{1}\left(\Omega_{1} \backslash \Omega\right)}+\left\|v \cdot \nabla_{x} f\right\|_{L^{1}\left(\Omega_{1} \backslash \Omega\right)} .
$$

Remark 2.3. Since, as proved in [9], page 194, eq. (3.8), $\left|P_{\gamma}^{\mathfrak{u}} f\right|_{2, \pm} \lesssim\left|P_{\gamma}^{\mathfrak{u}} f \mathbf{1}_{\gamma_{ \pm}^{\delta}}\right|_{2, \pm}$ and $\delta^{\theta / 2} \lesssim$ $\nu^{\frac{1}{2}} \lesssim \delta^{-\theta / 2}$, from previous lemma applied to $\nu f^{2}$ we get

$$
\left|P_{\gamma}^{\mathfrak{u}} f\right|_{2, \pm} \lesssim_{\delta}\|f\|_{L^{2}\left(\Omega_{1} \backslash \Omega\right)}+\left\|\nu^{-\frac{1}{2}} v \cdot \nabla f\right\|_{L^{2}\left(\Omega_{1} \backslash \Omega\right)} .
$$

Next two lemmas are useful to bound the boundary terms in the energy inequality: 


\section{Lemma 2.4.}

$$
\begin{gathered}
\left.\left|\int_{\partial \Omega} \mathrm{d} S \int_{\{v \cdot n>0\}} \mathrm{d} v v \cdot n\right| P_{\gamma}^{\mathfrak{u}} f\right|^{2}-\left.\int_{\partial \Omega} d S \int_{\{v \cdot n<0\}} \mathrm{d} v|v \cdot n|\left|P_{\gamma}^{\mathfrak{u}} f\right|^{2}|\lesssim \varepsilon| \mathfrak{u}\left|\int_{\gamma_{+}}\right| f\right|^{2} \mathrm{~d} \gamma . \\
\left|\int_{\partial \Omega} \mathrm{d} S \int_{\{v \cdot n>0\}} \mathrm{d} v v \cdot n P_{\gamma}^{\mathfrak{u}} f\left(1-P_{\gamma}^{\mathfrak{u}}\right) f\right| \lesssim \varepsilon|\mathfrak{u}| \int_{\gamma_{+}}|f|^{2} \mathrm{~d} \gamma .
\end{gathered}
$$

Proof. From the definition of $P_{\gamma}^{\mathfrak{u}}$,

$$
\int_{\{v \cdot n \gtrless 0\}} \mathrm{d} v|v \cdot n|\left|P_{\gamma}^{\mathfrak{u}} f\right|^{2}=\sqrt{2 \pi}\left|z_{\gamma}(f)\right|^{2} \int_{\{v \cdot n \gtrless 0\}} \mathrm{d} v \sqrt{2 \pi} \mu^{2} \mu_{\mathfrak{u}}^{-1}|v \cdot n| .
$$

Since by (1.32)

$$
\begin{aligned}
& \mu^{2} \mu_{\mathfrak{u}}^{-1}=\mu-\mu\left(\mu_{\mathfrak{u}}-\mu\right) \mu_{\mathfrak{u}}^{-1}=\mu-\mu\left[\varepsilon \mathfrak{u} \cdot v_{\mathfrak{u}} \mu_{\mathfrak{u}}+\varepsilon^{2} \phi_{\varepsilon} \sqrt{\mu_{\mathfrak{u}}} \mu_{\mathfrak{u}}^{-1}=\mu-\varepsilon \mu \mathfrak{u} \cdot v_{\mathfrak{u}}-\varepsilon^{2} \mu \phi_{\varepsilon} \mu_{\mathfrak{u}}^{-\frac{1}{2}},\right. \\
& \int_{\{v \cdot n \gtrless 0\}} \mathrm{d} v \sqrt{2 \pi} \mu^{2} \mu_{\mathfrak{u}}^{-1}|v \cdot n|=\int_{\{v \cdot n \gtrless 0\}} \mathrm{d} v \sqrt{2 \pi}|v \cdot n|\left[\mu-\varepsilon \mu \mathfrak{u} \cdot v_{\mathfrak{u}}-\varepsilon^{2} \mu \phi_{\varepsilon} \mu_{\mathfrak{u}}^{-\frac{1}{2}}\right] \\
& =1-\varepsilon \int_{\{v \cdot n \gtrless 0\}} \mathrm{d} v \sqrt{2 \pi}|v \cdot n| \mu \mathfrak{u} \cdot(v-\varepsilon \mathfrak{u})-\varepsilon^{2} \int_{\{v \cdot n \gtrless 0\}} \mathrm{d} v \sqrt{2 \pi} \mu|v \cdot n| \phi_{\varepsilon} \mid \mu \mu_{\mathfrak{u}}^{-\frac{1}{2}}=1+O(\varepsilon|\mathfrak{u}|) .
\end{aligned}
$$

The last term is bounded because, by (1.32), $\left|\phi_{\varepsilon}\right| \lesssim|\mathfrak{u}|^{2} \mu_{\mathfrak{u}}^{\frac{1}{2}^{-}}$. Therefore

$$
\int_{\{v \cdot n \gtrless 0\}} \mathrm{d} v\left|P_{\gamma} f\right|^{2}=\sqrt{2 \pi}\left|z_{\gamma}(f)\right|^{2}(1+O(\varepsilon|\mathfrak{u}|) .
$$

Thus

$$
\left.\left|\int_{\partial \Omega} \mathrm{d} S \int_{\{v \cdot n>0\}} \mathrm{d} v v \cdot n\right| P_{\gamma}^{\mathfrak{u}} f\right|^{2}-\left.\int_{\partial \Omega} \mathrm{d} S \int_{\{v \cdot n<0\}} \mathrm{d} v|v \cdot n|\left|P_{\gamma}^{\mathfrak{u}} f\right|^{2}\left|\lesssim O(\varepsilon|\mathfrak{u}|) \int_{\partial \Omega} \mathrm{d} S\right| z_{\gamma}(f)\right|^{2}
$$

and this proves (2.4), because

To prove (2.5) we note that

$$
\int_{\partial \Omega} \mathrm{d} S\left|z_{\gamma}(f)\right|^{2} \leq|f|_{2,+}^{2}
$$

$$
\begin{gathered}
\int_{\{v \cdot n>0\}} \mathrm{d} v v \cdot n P_{\gamma}^{\mathfrak{u}} f\left(1-P_{\gamma}^{\mathfrak{u}}\right) f=\int_{\{v \cdot n>0\}} \mathrm{d} v v \cdot n f P_{\gamma}^{\mathfrak{u}} f-\int_{\{v \cdot n>0\}} \mathrm{d} v v \cdot n\left|P_{\gamma}^{\mathfrak{u}} f\right|^{2} . \\
\int_{\{v \cdot n>0\}} \mathrm{d} v v \cdot n f P_{\gamma}^{\mathfrak{u}} f \\
=\sqrt{2 \pi} z_{\gamma}(f) \int_{\{v \cdot n>0\}} \mathrm{d} v v \cdot n f \mu \mu_{\mathfrak{u}}^{-\frac{1}{2}} \\
=\sqrt{2 \pi} z_{\gamma}(f) \int_{\{v \cdot n>0\}} \mathrm{d} v v \cdot n f\left[\mu_{\mathfrak{u}}^{\frac{1}{2}}+\left(\mu-\mu_{\mathfrak{u}}\right) \mu_{\mathfrak{u}}^{-\frac{1}{2}}\right] \\
=\sqrt{2 \pi}\left|z_{\gamma}(f)\right|^{2}+\sqrt{2 \pi}\left|z_{\gamma}(f)\right| \int_{\{v \cdot n>0\}} \mathrm{d} v v \cdot n f\left(\mu-\mu_{\mathfrak{u}}\right) \mu_{\mathfrak{u}}^{-\frac{1}{2}}
\end{gathered}
$$

Using again $\left(\mu-\mu_{\mathfrak{u}}\right) \mu_{\mathfrak{u}}^{-\frac{1}{2}}=\varepsilon \mathfrak{u} \cdot v_{\mathfrak{u}} \mu_{\mathfrak{u}}^{\frac{1}{2}}+\varepsilon^{2} \phi_{\varepsilon}$,

$$
\begin{array}{r}
\int_{\{v \cdot n>0\}} \mathrm{d} v v \cdot n f\left(\mu-\mu_{\mathfrak{u}}\right) \mu_{\mathfrak{u}}^{-\frac{1}{2}} \leq \varepsilon|\mathfrak{u}|\left(\int_{\{v \cdot n>0\}} \mathrm{d} v v \cdot n f^{2}\right)^{\frac{1}{2}}\left(\int_{\{v \cdot n>0\}} \mathrm{d} v v \cdot n\left[\left|v_{\mathfrak{u}}\right|^{2} \mu_{\mathfrak{u}}+\varepsilon|\mathfrak{u}|^{-2}\left|\phi_{\varepsilon}\right|^{2}\right]\right)^{\frac{1}{2}} \\
\lesssim \varepsilon|\mathfrak{u}|\left(\int_{\{v \cdot n>0\}} \mathrm{d} v v \cdot n f^{2}\right)^{\frac{1}{2}}
\end{array}
$$

Therefore

$$
\left|\int_{\partial \Omega} \mathrm{d} S \int_{\{v \cdot n>0\}} \mathrm{d} v v \cdot n P_{\gamma}^{\mathfrak{u}} f\left(1-P_{\gamma}^{\mathfrak{u}}\right) f\right| \lesssim \varepsilon|\mathfrak{u}| \int_{\partial \Omega} \mathrm{d} S\left|z_{\gamma}(f)\right|\left(\int_{\{v \cdot n>0\}} \mathrm{d} v v \cdot n f^{2}\right)^{\frac{1}{2}} \leq \varepsilon|\mathfrak{u}||f|_{2,+}^{2},
$$

and this concludes the proof. 
Lemma 2.5. For any $\eta>0$,

$$
\left|\int_{\partial \Omega} \mathrm{d} S \int_{\{v \cdot n<0\}} \mathrm{d} v\right| v \cdot n\left|\varepsilon^{\frac{1}{2}} r P_{\gamma}^{\mathfrak{u}} f\right| \lesssim \frac{1}{\eta}\left\|z_{\gamma}(r)\right\|_{2}^{2}+\varepsilon \eta|f|_{2,+}^{2}+\varepsilon^{\frac{3}{2}}|r|_{2,-}^{2} .
$$

Proof. We note that

$$
\begin{aligned}
\varepsilon^{\frac{1}{2}} \int_{\{v \cdot n<0\}} r P_{\gamma}^{\mathfrak{u}} f \mathrm{~d} v|v \cdot n| & =\varepsilon^{\frac{1}{2}} \sqrt{2 \pi} z_{\gamma}(f) \int_{\{v \cdot n<0\}} \mathrm{d} v r|v \cdot n| \mu \mu_{\mathfrak{u}}^{-\frac{1}{2}} \\
& =\varepsilon^{\frac{1}{2} \sqrt{2 \pi}} z_{\gamma}(f) z_{\gamma}(r)+\varepsilon^{\frac{1}{2}} \sqrt{2 \pi} z_{\gamma}(f) \int_{\{v \cdot n<0\}} \mathrm{d} v r|v \cdot n|\left(\mu-\mu_{\mathfrak{u}}\right) \mu_{\mathfrak{u}}^{-\frac{1}{2}}
\end{aligned}
$$

The integral on $\partial \Omega$ of the first term is bounded by

$$
\begin{aligned}
\varepsilon^{\frac{1}{2}}\left|\sqrt{2 \pi} \int_{\partial \Omega} \mathrm{d} S\right| z_{\gamma}(r)\left|z_{\gamma}(f)\right| \leq \frac{2 \pi}{4 \eta} \int_{\partial \Omega} \mathrm{d} S\left|z_{\gamma}(r)\right|^{2}+\eta \varepsilon \int_{\partial \Omega} \mathrm{d} S\left|z_{\gamma}(f)\right|^{2} & \\
& \left.\lesssim \eta^{-1}\left\|z_{\gamma}(r)\right\|_{2}^{2}+\varepsilon \eta|f|_{2,+}^{2}\right] .
\end{aligned}
$$

The second by is bounded by

$$
\begin{aligned}
\left|\varepsilon^{\frac{1}{2}} \int_{\partial \Omega} \mathrm{d} S\right| z_{\gamma}(f)\left|\int_{\{v \cdot n<0\}} \mathrm{d} v r\right| v \cdot n\left|\left(\mu-\mu_{\mathfrak{u}}\right) \mu_{\mathfrak{u}}^{-\frac{1}{2}}\right| & \\
& \lesssim \varepsilon^{\frac{3}{2}}|\mathfrak{u}| \int_{\partial \Omega} \mathrm{d} S\left(\int_{\{v \cdot n<0\}} \mathrm{d} v|v \cdot n||r|^{2}\right)^{\frac{1}{2}}\left|z_{\gamma}(f)\right| \leq \varepsilon^{\frac{3}{2}}|\mathfrak{u}|\left(|r|_{2,-}^{2}+|f|_{2,+}^{2}\right)
\end{aligned}
$$

and we obtain (2.7).

For fixed $\varepsilon$ the construction of the solution to the linear problem (1.42) is standard, see e.g. 24. To prove Theorem 1.5, we begin with the energy inequality.

Proposition 2.6. For $|\mathfrak{u}|$ sufficiently small the solution to (1.42) satisfies the inequality

$$
\begin{aligned}
\varepsilon^{-2}\left\|\left(\mathbf{I}-\mathbf{P}_{\mathfrak{u}}\right) f\right\|_{\nu}^{2}+\varepsilon^{-1}\left|\left(1-P_{\gamma}^{\mathfrak{u}}\right) f\right|_{2,+}^{2} \lesssim & \left\|\nu^{-\frac{1}{2}}\left(\mathbf{I}-\mathbf{P}_{\mathfrak{u}}\right) g\right\|_{2}^{2}+|\mathfrak{u}|^{2}\left\|\mathbf{P}_{\mathfrak{u}} f\right\|_{6}^{2}+\left(1+\varepsilon^{\frac{1}{2}}\right)|r|_{2,-}^{2} \\
& +(\varepsilon|\mathfrak{u}|)^{-1}\left\|z_{\gamma}(r)\right\|_{2}^{2}+\varepsilon^{-2}|\mathfrak{u}|^{-2}\left\|\mathbf{P}_{\mathfrak{u}} g\right\|_{\frac{6}{5}}^{2}+\left\|\mathbf{P}_{\mathfrak{u}} g\right\|_{2}^{2} .
\end{aligned}
$$

Proof. Use (2.1) with $h=f$. Then, multiplying by $\varepsilon^{-1}$ we have

$$
\frac{1}{2} \varepsilon^{-1} \int_{\gamma_{+}} \mathrm{d} \gamma f^{2}-\frac{1}{2} \varepsilon^{-1} \int_{\gamma_{-}} \mathrm{d} \gamma f^{2}+\varepsilon^{-2} \int_{\Omega^{c} \times \mathbb{R}^{3}} \mathrm{~d} x \mathrm{~d} v f L_{\mathfrak{u}} f-\varepsilon^{-1} \int_{\Omega^{c} \times \mathbb{R}^{3}} \mathrm{~d} x \mathrm{~d} v f g=0 .
$$

We use the spectral inequality (see e.g. [7], Th. 7.2.5),

$$
\varepsilon^{-2} \int_{\Omega^{c} \times \mathbb{R}^{3}} \mathrm{~d} x \mathrm{~d} v f L_{\mathfrak{u}} f \gtrsim \varepsilon^{-2}\left\|\left(\mathbf{I}-\mathbf{P}_{\mathfrak{u}}\right) f\right\|_{\nu}^{2} .
$$

Moreover, using the Holder inequality to bound $\left|\left(\mathbf{P}_{\mathfrak{u}} f, \mathbf{P}_{\mathfrak{u}} g\right)\right| \leq\left\|\mathbf{P}_{\mathfrak{u}} f\right\|_{6}\left\|\mathbf{P}_{\mathfrak{u}} g\right\|_{\frac{6}{5}}$,

$$
\begin{aligned}
\varepsilon^{-1}\left|\int_{\Omega^{c} \times \mathbb{R}^{3}} \mathrm{~d} x \mathrm{~d} v f g\right| \leq \varepsilon^{-1}\left\|\left(\mathbf{I}-\mathbf{P}_{\mathfrak{u}}\right) f\right\|_{\nu}\left\|\nu^{-\frac{1}{2}}\left(\mathbf{I}-\mathbf{P}_{\mathfrak{u}}\right) g\right\|_{2}+\varepsilon^{-1}\left\|\mathbf{P}_{\mathfrak{u}} f\right\|_{6}\left\|\mathbf{P}_{\mathfrak{u}} g\right\|_{\frac{6}{5}} \leq \\
\eta_{1} \varepsilon^{-2}\left\|\left(\mathbf{I}-\mathbf{P}_{\mathfrak{u}}\right) f\right\|_{\nu}^{2}+\frac{1}{4 \eta_{1}}\left\|\nu^{-\frac{1}{2}}\left(\mathbf{I}-\mathbf{P}_{\mathfrak{u}}\right) g\right\|_{2}^{2}+\eta_{2}\left\|\mathbf{P}_{\mathfrak{u}} f\right\|_{6}^{2}+\frac{1}{4 \eta_{2} \varepsilon^{2}}\left\|\mathbf{P}_{\mathfrak{u}} g\right\|_{\frac{6}{5}}^{2} .
\end{aligned}
$$

From the boundary conditions, on $\gamma_{-}$we have $f=P_{\gamma}^{\mathfrak{u}} f+\varepsilon^{\frac{1}{2}} r$. Hence, using Lemma 2.5

$$
\begin{array}{r}
\varepsilon^{-1} \int_{\gamma_{-}} \mathrm{d} \gamma f^{2}=\varepsilon^{-1} \int_{\gamma_{-}} \mathrm{d} \gamma\left[P_{\gamma}^{\mathfrak{u}} f+\varepsilon^{\frac{1}{2}} r\right]^{2}=\varepsilon^{-1} \int_{\gamma_{-}} \mathrm{d} \gamma\left(\left|P_{\gamma}^{\mathfrak{u}} f\right|^{2}+\varepsilon|r|^{2}+2 \varepsilon^{\frac{1}{2}} r P_{\gamma}^{\mathfrak{u}} f\right) \\
=\varepsilon^{-1}\left[\int_{\gamma_{-}} \mathrm{d} \gamma\left|P_{\gamma}^{\mathfrak{u}} f\right|^{2}+\varepsilon|r|_{2,-}^{2}+\varepsilon^{\frac{3}{2}}|r|_{2,-}^{2}+\frac{1}{\eta}\left\|z_{\gamma}(r)\right\|_{2}^{2}+\varepsilon \eta|f|_{2,+}^{2}\right] .
\end{array}
$$


Moreover

$$
\varepsilon^{-1} \int_{\gamma_{+}} \mathrm{d} \gamma f^{2}=\varepsilon^{-1} \int_{\gamma_{+}} \mathrm{d} \gamma\left[\left(1-P_{\gamma}^{\mathfrak{u}}\right) f\right]^{2}+\varepsilon^{-1} \int_{\gamma_{+}} \mathrm{d} \gamma\left[P_{\gamma}^{\mathfrak{u}} f\right]^{2}+2 \varepsilon^{-1} \int_{\gamma_{+}} \mathrm{d} \gamma\left[\left(1-P_{\gamma}^{\mathfrak{u}}\right) f\right]\left[P_{\gamma}^{\mathfrak{u}} f\right] .
$$

The last term is bounded by (2.5) and the second is replaced by $\int_{\gamma_{-}}\left[P_{\gamma}^{\mathfrak{u}} f\right]^{2}$ by using (2.4). Then $(|\mathfrak{u}|+\eta)|f|_{2,+}^{2}$ is split into $(|\mathfrak{u}|+\eta)\left|\left(1-P_{\gamma}^{\mathfrak{u}}\right) f\right|_{2,+}^{2}+(|\mathfrak{u}|+\eta)\left|P_{\gamma}^{\mathfrak{u}} f\right|_{2,+}^{2}$ Collecting the terms and choosing $\eta=|\mathfrak{u}|, \eta_{1}$ sufficiently small and $\eta_{2}=|\mathfrak{u}|^{2}$ we have the energy inequality

$$
\begin{aligned}
& \varepsilon^{-2}\left\|\left(\mathbf{I}-\mathbf{P}_{\mathfrak{u}}\right) f\right\|_{\nu}^{2}+\varepsilon^{-1}\left|\left(1-P_{\gamma}^{\mathfrak{u}}\right) f\right|_{2,+} \\
\lesssim & \left\|\nu^{-\frac{1}{2}}\left(\mathbf{I}-\mathbf{P}_{\mathfrak{u}}\right) g\right\|_{2}^{2}+\frac{1}{\varepsilon^{2}|\mathfrak{u}|^{2}}\left\|\mathbf{P}_{\mathfrak{u}} g\right\|_{\frac{6}{5}}^{2}+\left(1+\varepsilon^{\frac{1}{2}}\right)|r|_{2,-}^{2}+(\varepsilon|\mathfrak{u}|)^{-1}\left\|z_{\gamma}\right\|_{2}^{2}+\left|\mathfrak{u}\left\|\left.P_{\gamma}^{\mathfrak{u}} f\right|_{2,+} ^{2}+|\mathfrak{u}|^{2}\right\| \mathbf{P}_{\mathfrak{u}} f \|_{6}^{2},\right.
\end{aligned}
$$

where we have used $\int_{\gamma_{+}}\left[\left(1-P_{\gamma}^{\mathfrak{u}}\right) f\right]^{2}-|\mathfrak{u}|\left|\left(1-P_{\gamma}^{\mathfrak{u}}\right) f\right|_{2,+}^{2} \gtrsim\left|\left(1-P_{\gamma}^{\mathfrak{u}}\right) f\right|_{2,+}^{2}$ for $|\mathfrak{u}|$ sufficiently small. Next we use (2.3) to bound

$$
|\mathfrak{u}|\left|P_{\gamma}^{\mathfrak{u}} f\right|_{2,+}^{2} \leq|\mathfrak{u}|\|f\|_{L^{2}\left(\Omega_{1} \backslash \Omega\right)}^{2}+|\mathfrak{u}|\left\|\varepsilon^{-1}\left(\mathbf{I}-\mathbf{P}_{\mathfrak{u}}\right) f\right\|_{L^{2}\left(\Omega_{1} \backslash \Omega\right)}^{2}+|\mathfrak{u}|\left\|\nu^{-\frac{1}{2}} g\right\|_{L^{2}\left(\Omega_{1} \backslash \Omega\right)}^{2}
$$

Moreover, we split $\|f\|_{L^{2}\left(\Omega_{1} \backslash \Omega\right)}^{2}=\left\|\left(\mathbf{I}-\mathbf{P}_{\mathfrak{u}}\right) f\right\|_{L^{2}\left(\Omega_{1} \backslash \Omega\right)}^{2}+\left\|\mathbf{P}_{\mathfrak{u}} f\right\|_{L^{2}\left(\Omega_{1} \backslash \Omega\right)}^{2}$ and bound

$$
\left\|\mathbf{P}_{\mathfrak{u}} f\right\|_{L^{2}\left(\Omega_{1} \backslash \Omega\right)}^{2} \lesssim \Omega_{1}\left\|\mathbf{P}_{\mathfrak{u}} f\right\|_{6}^{2} .
$$

Finally, we bound

$$
\left\|\nu^{-\frac{1}{2}} g\right\|_{L^{2}\left(\Omega_{1} \backslash \Omega\right)}^{2} \lesssim\left\|\nu^{-\frac{1}{2}}\left(\mathbf{I}-\mathbf{P}_{\mathfrak{u}}\right) g\right\|_{2}^{2}+\left\|\mathbf{P}_{\mathfrak{u}} g\right\|_{2}^{2}
$$

We have so proved (2.8).

Proposition 2.7. Let $w=e^{\beta^{\prime}|v|^{2}}\langle v\rangle^{\beta}$. Then, for $0 \leq \beta^{\prime} \ll 1 / 4$ and $\beta \geq 0$ we have

$$
\varepsilon^{\frac{1}{2}}\|w f\|_{L^{\infty}\left(\Omega^{c}\right)} \lesssim \varepsilon^{-1}\left\|\left(\mathbf{I}-\mathbf{P}_{\mathfrak{u}}\right) f\right\|_{\nu}+\left\|\mathbf{P}_{\mathfrak{u}} f\right\|_{6}+\varepsilon^{\frac{1}{2}}|w r|_{\infty}+\varepsilon^{\frac{3}{2}}\left\|\langle v\rangle^{-1} w g\right\|_{\infty} .
$$

Proof. As in [10], Prop. 2.6.

\section{3. $L^{6}$ estimate of $\mathbf{P}_{\mathfrak{u}} f$}

Given $g$ and $r$, we consider the weak version of the linear problem (1.42): for any test function $\psi$,

$$
\begin{aligned}
& \int_{\gamma_{+}} \mathrm{d} \gamma f \psi-\int_{\Omega^{c} \times \mathbb{R}^{3}} \mathrm{~d} x \mathrm{~d} v f v \cdot \nabla \psi+\varepsilon^{-1} \int_{\Omega^{c} \times \mathbb{R}^{3}} \mathrm{~d} x \mathrm{~d} v \psi L_{\mathfrak{u}} f \\
&=\int_{\Omega^{c} \times \mathbb{R}^{3}} \mathrm{~d} x \mathrm{~d} v g \psi+\int_{\gamma_{-}} \mathrm{d} \gamma\left(P_{\gamma}^{\mathfrak{u}} f+\varepsilon^{\frac{1}{2}} r\right) \psi .
\end{aligned}
$$

Remind that $\mathbf{P}_{\mathfrak{u}} f=\sqrt{\mu_{\mathfrak{u}}}\left[a+b \cdot v_{\mathfrak{u}}+\frac{1}{2}\left(\left|v_{\mathfrak{u}}\right|^{2}-3\right)\right]$. To get a $L^{6}$ bound on $\mathbf{P}_{\mathfrak{u}} f$ we bound separately the functions $a, b$ and $c$ by means of suitable choices of the test functions $\psi$. To this end we will need to solve $-\Delta \phi=h \in L^{6 / 5}\left(\Omega^{c}\right)$ with Dirichlet or Neumann boundary conditions.

Lemma 3.1. For exterior domain $\Omega^{c}$ with $C^{2}$ boundary $\partial \Omega$, there exists a unique solution to $-\Delta \phi=h \in L^{6 / 5}\left(\Omega^{c}\right)$ with either Dirichlet or Neumann boundary conditions such that

$$
\|\nabla \phi\|_{L^{2}}+\|\phi\|_{L^{6}}+\left\|\nabla^{2} \phi\right\|_{L^{6 / 5}} \leq\|h\|_{L^{6 / 5}}
$$

Proof. We solve $-\Delta \phi=f \in L^{6 / 5}\left(\Omega^{c}\right)$ by the Lax-Milgram theorem: define a bilinear form

$$
[[\nabla \phi, \nabla \psi]] \equiv \int_{\Omega^{c}} \mathrm{~d} x \mathrm{~d} v \nabla \phi \cdot \nabla \psi
$$

with the functional $h$ defined by

$$
\langle h, \psi\rangle \equiv \int_{\Omega^{c}} \mathrm{~d} x \mathrm{~d} v f \psi .
$$

We choose homogeneous Sobolev space $\dot{H}^{1}\left(\Omega^{c}\right)$, with norm $\|\phi\|_{\dot{H}^{1}\left(\Omega^{c}\right)}=\|\nabla \phi\|_{L^{2}\left(\Omega^{c}\right)}$ for Neumnann boundary conditions and $\dot{H}_{0}^{1}\left(\Omega^{c}\right)$ for Dirichlet boundary conditions. 
We have the Sobolev embedding

$$
\|\xi\|_{L^{6}\left(\Omega^{c}\right)} \lesssim\|\nabla \xi\|_{L^{2}\left(\Omega^{c}\right)}
$$

(see 11], p. 263). Therefore $\langle h, \psi\rangle$ defines a bounded linear functional in $\dot{H}^{1}\left(\Omega^{c}\right)$ thanks to the inequality

$$
\langle h, \psi\rangle=\int_{\Omega^{c}} \mathrm{~d} x \mathrm{~d} v f \psi \leq\|f\|_{L^{6 / 5}}\|\psi\|_{L^{6}} \leq c_{h}\|\nabla \psi\|_{L^{2}}
$$

The existence and uniqueness as well as the first two inequalities then follows from Lax-Milgram theorem. To bound $\left\|\nabla^{2} \phi\right\|_{L^{6 / 5}}$, we take a smooth cutoff function $\chi$ such that

$$
\Delta(\chi \phi)=\chi h+2 \nabla \chi \cdot \nabla \phi+\Delta \chi \phi \in L^{6 / 5} .
$$

If $\chi$ is zero near $\partial \Omega$, then, by the $W^{2, p}$ estimate for the whole space, and the fact $\nabla \chi$ has compact support,

$$
\begin{aligned}
\left\|\nabla^{2} \chi \phi\right\|_{L^{6 / 5}} & \leq\|\chi h+2 \nabla \chi \cdot \nabla \phi+\Delta \chi \phi\|_{L^{6 / 5}} \\
& \leq\|h\|_{L^{6 / 5}} .
\end{aligned}
$$

On the other hand, if $\chi$ is zero for $|x|$ large, then by the $W^{2, p}$ estimate for mixed Dirichlet-Neumann b.c. in a fixed domain, we have

$$
\begin{aligned}
\left\|\nabla^{2}(\chi \phi)\right\|_{L^{6 / 5}} & \leq\|\chi h+2 \nabla \chi \cdot \nabla \phi+\Delta \chi \phi\|_{L^{6 / 5}}+\|\chi \phi\|_{L^{6 / 5}} \\
& \leq\|h\|_{L^{6 / 5}} .
\end{aligned}
$$

We therefore conclude (3.2).

Proposition 3.2. If $|\mathfrak{u}|$ is sufficiently small we have:

$$
\begin{aligned}
\left\|\mathbf{P}_{\mathfrak{u}} f\right\|_{6} \lesssim \varepsilon^{-1}\left\|\left(\mathbf{I}-\mathbf{P}_{\mathfrak{u}}\right) f\right\|_{\nu}+\left\|\left(\mathbf{I}-\mathbf{P}_{\mathfrak{u}}\right) f\right\|_{6}+\left\|g \nu^{-\frac{1}{2}}\right\|_{2}+\varepsilon^{-\frac{1}{2}} \mid & \left.\left(1-P_{\gamma}^{\mathfrak{u}}\right) f\right|_{2,+} \\
& +\varepsilon^{\frac{1}{2}}|r|_{\infty}+o(1)\left[\varepsilon^{\frac{1}{2}}\|f\|_{\infty}\right] .
\end{aligned}
$$

Remark 3.3. Note that

$$
\begin{array}{r}
\left\|\left(\mathbf{I}-\mathbf{P}_{\mathfrak{u}}\right) f\right\|_{6} \leq\left\|\left(\mathbf{I}-\mathbf{P}_{\mathfrak{u}}\right) f\right\|_{2}^{1 / 3}\left\|\left(\mathbf{I}-\mathbf{P}_{\mathfrak{u}}\right) f\right\|_{\infty}^{2 / 3}=\varepsilon^{1 / 3}\left\|\varepsilon^{-1}\left(\mathbf{I}-\mathbf{P}_{\mathfrak{u}}\right) f\right\|_{2}^{1 / 3} \varepsilon^{-\frac{1}{2} \times \frac{2}{3}}\left\|\varepsilon^{\frac{1}{2}}\left(\mathbf{I}-\mathbf{P}_{\mathfrak{u}}\right) f\right\|_{\infty}^{2 / 3} \\
\lesssim \eta\left\|\varepsilon^{\frac{1}{2}}\left(\mathbf{I}-\mathbf{P}_{\mathfrak{u}}\right) f\right\|_{\infty}+\frac{1}{\eta}\left\|\varepsilon^{-1}\left(\mathbf{I}-\mathbf{P}_{\mathfrak{u}}\right) f\right\|_{2} .
\end{array}
$$

Therefore by choosing $\eta$ small we obtain

$$
\left\|\mathbf{P}_{\mathfrak{u}} f\right\|_{6} \lesssim \varepsilon^{-1}\left\|\left(\mathbf{I}-\mathbf{P}_{\mathfrak{u}}\right) f\right\|_{\nu}+\left\|g \nu^{-\frac{1}{2}}\right\|_{2}+\varepsilon^{-\frac{1}{2}}\left|\left(1-P_{\gamma}^{\mathfrak{u}}\right) f\right|_{2,+}+\varepsilon^{\frac{1}{2}}|r|_{\infty}+o(1)\left[\varepsilon^{\frac{1}{2}}\|f\|_{\infty}\right] .
$$

Proof.

Step 1:

In order to get a bound for $c$, we choose the function $\psi_{c}$ in (3.1) as

$$
\psi_{c}=\sqrt{\mu_{\mathfrak{u}}}\left(\left|v_{\mathfrak{u}}\right|^{2}-\beta_{c}\right) v_{\mathfrak{u}} \cdot \nabla \varphi_{c},
$$

with $\beta_{c}$ a suitable constant to be chosen later and $\varphi_{c}$ solution to the problem

$$
-\Delta \varphi_{c}=c^{5} \text { in } \Omega^{c}, \quad \varphi=0 \text { on } \partial \Omega .
$$

Hence, by previous discussion, there is a unique $\varphi_{c}$ and

$$
\left\|\nabla \varphi_{c}\right\|_{\dot{H}^{1}\left(\Omega^{c}\right)} \leq\left\|c^{5}\right\|_{L^{\frac{6}{5}}\left(\Omega^{c}\right)}=\|c\|_{L^{6}\left(\Omega^{c}\right)}^{5} .
$$

We start computing the term $\int_{\Omega^{c} \times \mathbb{R}^{3}} d x d v f v \cdot \nabla \psi_{c}$. We have:

$$
\int_{\Omega^{c} \times \mathbb{R}^{3}} \mathrm{~d} x \mathrm{~d} v f v \cdot \nabla \psi_{c}=\int_{\Omega^{c} \times \mathbb{R}^{3}} \mathrm{~d} x \mathrm{~d} v f v_{\mathfrak{u}} \cdot \nabla \psi_{c}+\varepsilon \int_{\Omega^{c} \times \mathbb{R}^{3}} \mathrm{~d} x \mathrm{~d} v f \mathfrak{u} \cdot \nabla \psi_{c} .
$$

By (3.6),$f=\mathbf{P}_{\mathfrak{u}} f+\left(\mathbf{I}-\mathbf{P}_{\mathfrak{u}}\right) f$ and the Young inequality,

$$
\left|\varepsilon \int_{\Omega^{c} \times \mathbb{R}^{3}} \mathrm{~d} x \mathrm{~d} v f \mathfrak{u} \cdot \nabla \psi_{c}\right| \leq \varepsilon|\mathfrak{u}|\|c\|_{6}^{5}\|f\|_{6} \lesssim \varepsilon|\mathfrak{u}|\left\|\mathbf{P}_{\mathfrak{u}} f\right\|_{6}^{6}+\varepsilon|\mathfrak{u}|\left\|\left(\mathbf{I}-\mathbf{P}_{\mathfrak{u}}\right) f\right\|_{6}^{6} .
$$


By using $f=\mathbf{P}_{\mathfrak{u}} f+\left(\mathbf{I}-\mathbf{P}_{\mathfrak{u}}\right) f$ and the expression of $\mathbf{P}_{\mathfrak{u}} f$, we need to compute

$$
\begin{gathered}
\int_{\Omega^{c} \times \mathbb{R}^{3}} \mathrm{~d} x \mathrm{~d} v a \sqrt{\mu_{\mathfrak{u}}} v_{\mathfrak{u}} \cdot \nabla \psi_{c} \\
\int_{\Omega^{c} \times \mathbb{R}^{3}} \mathrm{~d} x \mathrm{~d} v b \cdot v_{\mathfrak{u}} \sqrt{\mu_{\mathfrak{u}}} v_{\mathfrak{u}} \cdot \nabla \psi_{c} \\
\int_{\Omega^{c} \times \mathbb{R}^{3}} \mathrm{~d} x \mathrm{~d} v c \frac{\left|v_{\mathfrak{u}}\right|^{2}-3}{2} \sqrt{\mu_{\mathfrak{u}}} v_{\mathfrak{u}} \cdot \nabla \psi_{c} \\
\int_{\Omega^{c} \times \mathbb{R}^{3}} \mathrm{~d} x \mathrm{~d} v v \cdot \nabla \psi_{c}\left(\mathbf{I}-\mathbf{P}_{\mathfrak{u}}\right) f=\int_{\Omega^{c} \times \mathbb{R}^{3}} \mathrm{~d} x \mathrm{~d} v \sqrt{\mu_{\mathfrak{u}}}\left(\left|v_{\mathfrak{u}}\right|^{2}-\beta_{c}\right) v_{\mathfrak{u}} \otimes v_{\mathfrak{u}}: \nabla \otimes \nabla \varphi_{c}\left(\mathbf{I}-\mathbf{P}_{\mathfrak{u}}\right) f
\end{gathered}
$$

Using (3.6), by the Young inequality, the last one is bounded by

$$
\left\|\left(\mathbf{I}-\mathbf{P}_{\mathfrak{u}}\right) f\right\|_{6}\|c\|_{L^{6}\left(\Omega^{c}\right)}^{5} \leq \frac{5}{6} \eta\|c\|_{L^{6}\left(\Omega^{c}\right)}^{6}+\frac{1}{6} \eta^{-\frac{1}{5}}\left\|\left(\mathbf{I}-\mathbf{P}_{\mathfrak{u}}\right) f\right\|_{6}^{6},
$$

for any $\eta>0$.

With the choice $\beta_{c}=5$ it results

$$
\int_{\mathbb{R}^{3}} \mathrm{~d} v\left(\left|v_{\mathfrak{u}}\right|^{2}-\beta_{c}\right) v_{\mathfrak{u}} \otimes v_{\mathfrak{u}} \mu_{\mathfrak{u}}=0
$$

and the term in (3.7) vanishes. The term (3.8) vanishes because is odd in $v_{\mathfrak{u}}$. Next we compute the term (3.9). We have

$$
\int_{\mathbb{R}^{3}} \mathrm{~d} v v_{\mathfrak{u}} \otimes v_{\mathfrak{u}} \frac{\left|v_{\mathfrak{u}}\right|^{2}-3}{2}\left(\left|v_{\mathfrak{u}}\right|^{2}-\beta_{c}\right) \mu_{\mathfrak{u}}=5 \mathbf{I}
$$

Therefore

$$
\begin{gathered}
\int_{\Omega^{c} \times \mathbb{R}^{3}} \mathrm{~d} x \mathrm{~d} v c \frac{\left|v_{\mathfrak{u}}\right|^{2}-3}{2} \sqrt{\mu_{\mathfrak{u}}} v_{\mathfrak{u}} \cdot \nabla \psi_{c}=\int_{\Omega^{c}} \mathrm{~d} x c \nabla \otimes \nabla \varphi_{c}: \int_{\mathbb{R}^{3}} \mathrm{~d} v v_{\mathfrak{u}} \otimes v_{\mathfrak{u}} \frac{\left|v_{\mathfrak{u}}\right|^{2}-3}{2}\left(\left|v_{\mathfrak{u}}\right|^{2}-\beta_{c}\right) \mu_{\mathfrak{u}}= \\
5 \int_{\Omega^{c}} \mathrm{~d} x c \Delta \varphi_{c}=-5 \int_{\Omega^{c}} \mathrm{~d} x|c|^{6}=-5\|c\|_{L^{6}\left(\Omega^{c}\right)}^{6},
\end{gathered}
$$

because of (3.5). By (3.2) and Young inequality, we have

$\varepsilon^{-1}\left|\int_{\Omega^{c} \times \mathbb{R}^{3}} \mathrm{~d} x \mathrm{~d} v \psi_{c} L_{\mathfrak{u}} f\right| \leq \varepsilon^{-1}\left\|\nabla \varphi_{c}\right\|_{L^{2}\left(\Omega^{c}\right)}\left\|\left(\mathbf{I}-\mathbf{P}_{\mathfrak{u}}\right) f\right\|_{\nu} \leq \frac{5}{6} \eta\|c\|_{6}^{6}+\frac{1}{6}(4 \eta)^{-\frac{1}{5}}\left[\varepsilon^{-1}\left\|\left(\mathbf{I}-\mathbf{P}_{\mathfrak{u}}\right) f\right\|_{\nu}\right]^{6}$, for any $\eta>0$.

Similarly, we get

$$
\left|\int_{\Omega^{c} \times \mathbb{R}^{3}} \mathrm{~d} x \mathrm{~d} v \psi_{c} g\right| \lesssim\left\|\nabla \varphi_{c}\right\|_{L^{2}\left(\Omega^{c}\right)}\left\|g \nu^{-\frac{1}{2}}\right\|_{2} \leq \frac{5}{6} \eta\|c\|_{6}^{6}+\frac{1}{6}(4 \eta)^{-\frac{1}{5}}\left\|g \nu^{-\frac{1}{2}}\right\|_{2}^{6},
$$

for any $\eta>0$.

Next we compute the boundary terms. We decompose $f$ on $\gamma$ as $f=P_{\gamma}^{\mathfrak{u}} f+\mathbf{1}_{\gamma_{+}}\left(1-P_{\gamma}^{\mathfrak{u}}\right) f+$ $\mathbf{1}_{\gamma_{-}} \varepsilon^{\frac{1}{2}} r$.

First consider the term

$$
\int_{\gamma} \mathrm{d} \gamma P_{\gamma}^{\mathfrak{u}} f \psi_{c}=\int_{\partial \Omega} \mathrm{d} S(x) \nabla \varphi_{c} \cdot \int_{\mathbb{R}^{3}} \mathrm{~d} v(n \cdot v) v_{\mathfrak{u}}\left(\left|v_{\mathfrak{u}}\right|^{2}-\beta_{c}\right) \sqrt{\mu_{\mathfrak{u}}} P_{\gamma}^{\mathfrak{u}} f .
$$

From the expression of $P_{\gamma}^{\mathfrak{u}} f$ we see that

$$
\sqrt{\mu_{\mathfrak{u}}} P_{\gamma}^{\mathfrak{u}} f=\sqrt{2 \pi} \mu z_{\gamma}(f) .
$$

Therefore, we need to compute $\int_{\mathbb{R}^{3}} d v \int_{\mathbb{R}^{3}} d v(n \cdot v) v_{\mathfrak{u}}\left(\left|v_{\mathfrak{u}}\right|^{2}-\beta_{c}\right) \mu(v)$. We have

$$
v_{\mathfrak{u}}\left(|v-\varepsilon \mathfrak{u}|^{2}-\beta_{c}\right)=v\left(|v|^{2}-\beta_{c}\right)+\varepsilon\left(-\mathfrak{u}|v|^{2}-2 \mathfrak{u} \cdot v v+\beta_{c} \mathfrak{u}\right)+\varepsilon^{2}\left(|\mathfrak{u}|^{2} v+2 \mathfrak{u} \cdot v \mathfrak{u}\right)-\varepsilon^{3}|\mathfrak{u}|^{2} \mathfrak{u} .
$$


Since the terms of order $\varepsilon$ and $\varepsilon^{3}$ are even in $v$, after multiplication by $v \cdot n$, their contributions vanish (note that the integration in $v$ is on the full $\mathbb{R}^{3}$, not on $\{v \cdot n \lessgtr 0\}$. The contribution of the term of order 1 vanishes by the choice of $\beta_{c}$ (3.11), so we conclude that

$$
\int_{\gamma} \mathrm{d} \gamma P_{\gamma}^{\mathfrak{u}} f \psi_{c}=\varepsilon^{2} \int_{\partial \Omega} \mathrm{d} S(x) \nabla \varphi_{c} \cdot \int_{\mathbb{R}^{3}} \mathrm{~d} v(n \cdot v)\left(|\mathfrak{u}|^{2} v+2 \mathfrak{u} \cdot v \mathfrak{u}\right) \sqrt{\mu_{\mathfrak{u}}} P_{\gamma}^{\mathfrak{u}} f .
$$

We need the Sobolev trace theorem to bound $\nabla \varphi_{c}$ on $\partial \Omega$.

\section{Lemma 3.4.}

$$
\left\|\nabla \varphi_{c}\right\|_{L^{\frac{4}{3}(\partial \Omega)}} \leq\|c\|_{6}^{5} .
$$

Proof. If $\Omega$ is a $C^{1}$ domain in $\mathbb{R}^{N}$, we have the following trace estimate 21], p. 466:

$$
\left(\int_{\partial \Omega} \mathrm{d} S(x)|u|^{\frac{p(N-1)}{N-p}}\right)^{\frac{N-p}{p(N-1)}} \leq C(N, p)\left(\int_{\Omega_{1} \backslash \Omega} \mathrm{d} x|u|^{p}+\int_{\Omega_{1} \backslash \Omega} d x|\nabla u|^{p}\right)^{\frac{1}{p}} .
$$

This is a consequence of the trace theorem $W^{1, p}\left(\Omega_{1} \backslash \Omega\right) \rightarrow W^{1-\frac{1}{p}, p}\left(\partial\left(\Omega_{1} \backslash \Omega\right)\right)$, and the Sobolev embedding in $N-1$ dimensional sub-manifold $\left(W^{1-\frac{1}{p}, p}\left(\partial\left(\Omega_{1} \backslash \Omega\right)\right) \subset L^{\frac{p(N-1)}{N-p}}\left(\Omega_{1} \backslash \Omega\right)\right.$ for $\frac{N-p}{p(N-1)}=$ $\left.\frac{1}{p}-\frac{1-\frac{1}{p}}{N-1}\right)$. In particular, with $p=\frac{6}{5}$ and $N=3$ we have $\frac{p(N-1)}{N-p}=\frac{4}{3}$. With $u=\nabla \varphi_{c}$, we have

$$
\left\|\nabla_{x} \varphi_{c}\right\|_{L^{\frac{4}{3}(\partial \Omega)}} \lesssim\|c\|_{L^{6}\left(\Omega_{1} \backslash \Omega\right)}^{5} \leq\|c\|_{L^{6}\left(\Omega^{c}\right)}^{5} .
$$

Therefore, by Holder inequality,

$$
\left|\int_{\gamma} \mathrm{d} \gamma P_{\gamma}^{\mathfrak{u}} f \psi_{c}\right| \leq \varepsilon^{2}|\mathfrak{u}|^{2}\left\|\nabla_{x} \varphi_{c}\right\|_{L^{\frac{4}{3}(\partial \Omega)}}\left\|P_{\gamma}^{\mathfrak{u}} f\right\|_{L^{4}(\gamma)} .
$$

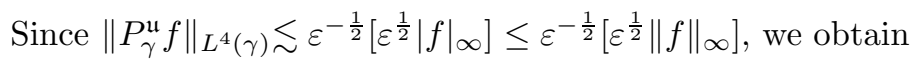

$$
\left|\int_{\gamma} \mathrm{d} \gamma P_{\gamma}^{\mathfrak{u}} f \psi_{c}\right| \lesssim \varepsilon^{2}|\mathfrak{u}|^{2} \varepsilon^{-\frac{1}{2}}\left[\varepsilon^{\frac{1}{2}}\|f\|_{\infty}\right]\|c\|_{6}^{5} \lesssim \varepsilon^{2} \varepsilon^{-\frac{1}{2}}|\mathfrak{u}|^{2} \frac{5}{6}\|c\|_{6}^{6}+\varepsilon^{2}|\mathfrak{u}|^{2} \frac{1}{6} \varepsilon^{-\frac{1}{2}}\left[\varepsilon^{\frac{1}{2}}\|f\|_{\infty}\right]^{6} .
$$

Next, we need to bound $\int_{\gamma} \mathbf{1}_{\gamma_{+}}\left(1-P_{\gamma}^{\mathfrak{u}}\right) f \psi_{c}$. We have

$$
\left|\int_{\gamma} \mathrm{d} \gamma \mathbf{1}_{\gamma_{+}}\left(1-P_{\gamma}^{\mathfrak{u}}\right) f \psi_{c}\right| \leq\left\|\nabla_{x} \varphi_{c}\right\|_{L^{\frac{4}{3}(\partial \Omega)}}\left\|\mathbf{1}_{\gamma_{+}}\left(1-P_{\gamma}^{\mathfrak{u}}\right) f\right\|_{L^{4}(\gamma)} .
$$

But

$$
\left\|\mathbf{1}_{\gamma_{+}}\left(1-P_{\gamma}^{\mathfrak{u}}\right) f\right\|_{L^{4}(\gamma)} \leq\left[\varepsilon^{-\frac{1}{2}}\left\|\mathbf{1}_{\gamma_{+}}\left(1-P_{\gamma}^{\mathfrak{u}}\right) f\right\|_{L^{2}(\gamma)}\right]^{\frac{1}{2}}\left[\varepsilon^{\frac{1}{2}}\left\|\mathbf{1}_{\gamma_{+}}\left(1-P_{\gamma}^{\mathfrak{u}}\right) f\right\|_{L^{\infty}(\gamma)}\right]^{\frac{1}{2}} .
$$

Thus, we conclude that, for any $\eta>0$ and $\eta^{\prime}>0$

$$
\left.\left|\int_{\gamma} \mathbf{1}_{\gamma_{+}}\left(1-P_{\gamma}^{\mathfrak{u}}\right) f \psi_{c} d \gamma\right| \lesssim \eta\|c\|_{6}^{6}+\eta^{\prime}\left[\varepsilon^{\frac{1}{2}}\|f\|_{\infty}\right]^{6}+C_{\eta, \eta^{\prime}}\left[\varepsilon^{-\frac{1}{2}}\left\|\mathbf{1}_{\gamma_{+}}\left(1-P_{\gamma}^{\mathfrak{u}}\right) f\right\|_{L^{2}(\gamma)}\right]^{6}\right\} .
$$

In conclusion the boundary terms are bounded, for any $\eta>0, \eta^{\prime}>0$, by

$$
\left.\left|\int_{\gamma} \mathrm{d} \gamma \psi_{c}\left[P_{\gamma}^{\mathfrak{u}} f+\mathbf{1}_{\gamma_{+}}\left(1-P_{\gamma}^{\mathfrak{u}}\right) f\right]\right| \leq \eta\|c\|_{6}^{6}+\eta^{\prime}\left[\varepsilon^{\frac{1}{2}}\|f\|_{\infty}\right]^{6}+\left.C_{\eta, \eta^{\prime}}\left[\varepsilon^{-\frac{1}{2}} \|\left(1-P_{\gamma}^{\mathfrak{u}}\right) f\right)\right|_{2 \cdot \gamma_{+}}\right]^{6} .
$$

Finally,

$$
\left|\int_{\gamma_{-}} \varepsilon^{\frac{1}{2}} \mathrm{~d} \gamma r \psi_{c}\right| \leq\left\|\nabla \varphi_{c}\right\|_{L^{4 / 3}(\partial \Omega)}\left\|\varepsilon^{\frac{1}{2}} r\right\|_{L^{4}(\partial \Omega)} \leq \varepsilon^{\frac{1}{2}}\|c\|_{6}^{5}|r|_{\infty} .
$$

By collecting all the terms and choosing $\eta$ and $\eta^{\prime}$ sufficiently small we conclude that

$$
\begin{aligned}
\|c\|_{6} \lesssim \varepsilon^{-1}\left\|\left(\mathbf{I}-\mathbf{P}_{\mathfrak{u}}\right) f\right\|_{\nu}+\left\|\left(\mathbf{I}-\mathbf{P}_{\mathfrak{u}}\right) f\right\|_{6} & +(\varepsilon|\mathfrak{u}|)^{\frac{1}{6}}\left\|\mathbf{P}_{\mathfrak{u}} f\right\|_{6}+\left\|g \nu^{-\frac{1}{2}}\right\|_{L^{2}\left(\Omega^{c} \times \mathbb{R}^{3}\right)} \\
& +\varepsilon^{-\frac{1}{2}}\left|\left(1-P_{\gamma}^{\mathfrak{u}}\right) f\right|_{2,+}+\varepsilon^{\frac{1}{2}}|r|_{\infty}+o(1)\left[\varepsilon^{\frac{1}{2}}\|f\|_{\infty}\right] .
\end{aligned}
$$

Step 2: 

$i, j$

In order to estimate $b$ we shall use two test functions. The first is chosen as follows: for fixed

$$
\psi=\psi_{b}^{i, j} \equiv\left(v_{\mathfrak{u}, i}^{2}-\beta_{b}\right) \sqrt{\mu_{\mathfrak{u}}} \partial_{j} \varphi_{b}^{j}, \quad i, j=1, \ldots, d,
$$

where $\beta_{b}$ is a constant to be determined, and

$$
-\Delta_{x} \varphi_{b}^{j}(x)=b_{j}^{5}(x),\left.\quad \varphi_{b}^{j}\right|_{\partial \Omega}=0 .
$$

As before, there is a unique $\varphi_{b}^{j}$ and

$$
\left\|\nabla \varphi_{b}^{j}\right\|_{\dot{H}^{1}\left(\Omega^{c}\right)} \leq\left\|\left|b_{j}\right|^{5}\right\|_{L^{\frac{6}{5}\left(\Omega^{c}\right)}}=\left\|b_{j}\right\|_{L^{6}\left(\Omega^{c}\right)}^{5} .
$$

We start computing the term $\int_{\Omega^{c} \times \mathbb{R}^{3}} d x d v f v \cdot \nabla \psi_{b}^{i, j}$. We have:

$$
\int_{\Omega^{c} \times \mathbb{R}^{3}} \mathrm{~d} x \mathrm{~d} v f v \cdot \nabla \psi_{b}^{i, j}=\int_{\Omega^{c} \times \mathbb{R}^{3}} \mathrm{~d} x \mathrm{~d} v f v_{\mathfrak{u}} \cdot \nabla \psi_{b}^{i, j}+\varepsilon \int_{\Omega^{c} \times \mathbb{R}^{3}} \mathrm{~d} x \mathrm{~d} v f \mathfrak{u} \cdot \nabla \psi_{b}^{i, j} .
$$

By (3.20) and the Young inequality,

$$
\left|\varepsilon \int_{\Omega^{c} \times \mathbb{R}^{3}} \mathrm{~d} x \mathrm{~d} v f \mathfrak{u} \cdot \nabla \psi_{b}^{i, j}\right| \leq \varepsilon|\mathfrak{u}|\left\|b_{j}\right\|_{6}^{5}\|f\|_{6} \leq \varepsilon|\mathfrak{u}|\left\|\mathbf{P}_{\mathfrak{u}} f\right\|_{6}^{6}+\varepsilon|\mathfrak{u}|\left\|\left(\mathbf{I}-\mathbf{P}_{\mathfrak{u}}\right) f\right\|_{6}^{6} .
$$

By using $f=\mathbf{P}_{\mathfrak{u}} f+\left(\mathbf{I}-\mathbf{P}_{\mathfrak{u}}\right) f$ and the expression of $\mathbf{P}_{\mathfrak{u}} f$, we need to compute

$$
\begin{gathered}
\int_{\Omega^{c} \times \mathbb{R}^{3}} \mathrm{~d} x \mathrm{~d} v a \sqrt{\mu_{\mathfrak{u}}} v_{\mathfrak{u}} \cdot \nabla \psi_{b}^{i, j} \\
\int_{\Omega^{c} \times \mathbb{R}^{3}} \mathrm{~d} x \mathrm{~d} v b \cdot v_{\mathfrak{u}} \sqrt{\mu_{\mathfrak{u}}} v_{\mathfrak{u}} \cdot \nabla \psi_{b}^{i, j} \\
\int_{\Omega^{c} \times \mathbb{R}^{3}} \mathrm{~d} x \mathrm{~d} v c \frac{\left|v_{\mathfrak{u}}\right|^{2}-3}{2} \sqrt{\mu_{\mathfrak{u}}} v_{\mathfrak{u}} \cdot \nabla \psi_{b}^{i, j} \\
\int_{\Omega^{c} \times \mathbb{R}^{3}} \mathrm{~d} x \mathrm{~d} v v \cdot \nabla \psi_{b}^{i, j}\left(\mathbf{I}-\mathbf{P}_{\mathfrak{u}}\right) f=\int_{\Omega \times \mathbb{R}^{3}} \mathrm{~d} x \mathrm{~d} v \sqrt{\mu_{\mathfrak{u}}}\left(\left|v_{\mathfrak{u}}\right|^{2}-\beta_{b}\right)^{2} v_{\mathfrak{u}} \otimes v_{\mathfrak{u}}: \nabla \otimes \nabla \varphi_{b}^{j}\left(\mathbf{I}-\mathbf{P}_{\mathfrak{u}}\right) f
\end{gathered}
$$

Using (3.20), the last one is bounded by

$$
\left\|\left(\mathbf{I}-\mathbf{P}_{\mathfrak{u}}\right)\right\|_{6}\left\|b_{j}\right\|_{L^{6}\left(\Omega^{c}\right)}^{5} \leq \frac{5}{6} \eta\left\|b_{j}\right\|_{L^{6}\left(\Omega^{c}\right)}^{6}+\frac{1}{6} \eta^{-\frac{1}{5}}\left\|\left(\mathbf{I}-\mathbf{P}_{\mathfrak{u}}\right)\right\|_{6}^{6},
$$

for any $\eta>0$. By oddness the terms in (3.21) and (3.23) vanish. We choose $\beta_{b}>0$ such that for all $i$,

$$
\int_{\mathbb{R}^{3}}\left[v_{\mathfrak{u}, i}^{2}-\beta_{b}\right] \mu_{\mathfrak{u}}(v) \mathrm{d} v=\frac{1}{\sqrt{2 \pi}} \int_{\mathbb{R}} \mathrm{d} v\left[v_{\mathfrak{u}, 1}^{2}-\beta_{b}\right] e^{-\frac{\left|v_{\mathfrak{u}, 1}\right|^{2}}{2}} \mathrm{~d} v_{1}=0,
$$

and we find $\beta_{b}=1$. Note that for such choice of $\beta_{b}$ and for $i \neq k$, by an explicit computation

$$
\begin{aligned}
\int_{\mathbb{R}^{3}}\left(v_{\mathfrak{u}, i}^{2}-\beta_{b}\right) v_{\mathfrak{u}, k}^{2} \mu_{\mathfrak{u}} \mathrm{d} v & =0, \\
\int_{\mathbb{R}^{3}}\left(v_{\mathfrak{u}, i}^{2}-\beta_{b}\right) v_{\mathfrak{u}, i}^{2} \mu \mathrm{d} v & =2 .
\end{aligned}
$$

As a consequence

$$
\begin{aligned}
\sum_{k, \ell} \int_{\Omega^{c} \times \mathbb{R}^{3}} \mathrm{~d} x \mathrm{~d} v b_{k} v_{\mathfrak{u}, k} \sqrt{\mu_{\mathfrak{u}}} v_{\mathfrak{u}, \ell}\left(v_{\mathfrak{u}, i}^{2}-\beta_{b}\right) \sqrt{\mu_{\mathfrak{u}}} \partial_{\ell} \partial_{j} \varphi_{b}^{j} & \\
& =\sum_{k, \ell} \delta_{k, \ell} \delta_{\ell, i} \int_{\Omega^{c}} \mathrm{~d} x b_{k} \partial_{\ell} \partial_{j} \varphi_{b}^{j}=\int_{\Omega^{c}} \mathrm{~d} x b_{i} \partial_{i} \partial_{j} \varphi_{b}^{j} .
\end{aligned}
$$

We have also

$\varepsilon^{-1}\left|\int_{\Omega^{c} \times \mathbb{R}^{3}} \mathrm{~d} x \mathrm{~d} v \psi_{b}^{i, j} L_{\mathfrak{u}} f\right| \leq \varepsilon^{-1}\left\|\nabla \varphi_{b}^{j}\right\|_{L^{2}\left(\Omega^{c}\right)}\left\|\left(\mathbf{I}-\mathbf{P}_{\mathfrak{u}}\right) f\right\|_{\nu} \leq \frac{5}{6} \eta\left\|b_{j}\right\|_{6}^{6}+\frac{1}{6} \eta^{-\frac{1}{5}}\left[\varepsilon^{-1}\left\|\left(\mathbf{I}-\mathbf{P}_{\mathfrak{u}}\right) f\right\|_{\nu}\right]^{6}$, for any $\eta>0$. 
Similarly, we get

$$
\left|\int_{\Omega^{c} \times \mathbb{R}^{3}} \mathrm{~d} x \mathrm{~d} v \psi_{b}^{i, j} g\right| \lesssim\left\|\nabla \varphi_{b_{j}}\right\|_{L^{2}\left(\Omega^{c}\right)}\left\|g \nu^{-\frac{1}{2}}\right\|_{2} \leq \frac{5}{6} \eta\left\|b_{j}\right\|_{6}^{6}+\frac{1}{6} \eta^{-\frac{1}{5}}\left\|g \nu^{-\frac{1}{2}}\right\|_{2}^{6},
$$

for any $\eta>0$.

Next we compute the boundary terms. We decompose $f$ on $\gamma$ as $f=P_{\gamma}^{\mathfrak{u}} f+\mathbf{1}_{\gamma_{+}}\left(1-P_{\gamma}^{\mathfrak{u}}\right) f+$

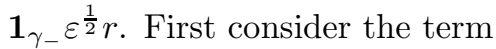

$$
\int_{\gamma} \mathrm{d} \gamma P_{\gamma}^{\mathfrak{u}} f \psi_{b}^{i, j}=\int_{\partial \Omega} \mathrm{d} S(x) \nabla \varphi_{b}^{j} \cdot \int_{\mathbb{R}^{3}} \mathrm{~d} v(n \cdot v)\left(\left|v_{\mathfrak{u}, i}\right|^{2}-\beta_{b}\right) \sqrt{\mu_{\mathfrak{u}}} P_{\gamma}^{\mathfrak{u}} f .
$$

Since $\sqrt{\mu_{\mathfrak{u}}} P_{\gamma}^{\mathfrak{u}} f=\sqrt{2 \pi} \mu z_{\gamma}(f)$, we need to compute $\int_{\mathbb{R}^{3}} d v \int_{\mathbb{R}^{3}} d v(n \cdot v)\left(v_{\mathfrak{u}, i}^{2}-\beta_{b}\right) \mu(v)$. We have

$$
v_{\mathfrak{u}, i}^{2}-\beta_{b}=v_{i}^{2}-\beta_{b}-2 \varepsilon \mathfrak{u}_{i} v_{i}+\varepsilon^{2} \mathfrak{u}_{i}^{2} .
$$

The terms of order 1 and $\varepsilon^{2}$ vanish by oddness. Therefore

$$
\int_{\gamma} P_{\gamma}^{\mathfrak{u}} \mathrm{d} \gamma f \psi_{b}^{i, j}=-\varepsilon \int_{\partial \Omega} \mathrm{d} S(x) \nabla \varphi_{b}^{j} \cdot \int_{\mathbb{R}^{3}} \mathrm{~d} v(n \cdot v) 2 \mathfrak{u}_{i} v_{i} \sqrt{\mu_{\mathfrak{u}}} P_{\gamma}^{\mathfrak{u}} f
$$

Thus, by using Lemma 3.4 .

$$
\left|\int_{\gamma} \mathrm{d} \gamma P_{\gamma}^{\mathfrak{u}} f \psi_{b}^{i, j}\right| \leq \varepsilon|\mathfrak{u}| \varepsilon^{-\frac{1}{2}}\left[\varepsilon^{\frac{1}{2}}\|f\|_{\infty}\right]\left\|b_{j}\right\|_{6}^{5} \lesssim \varepsilon^{\frac{1}{2}}|\mathfrak{u}|\left\|b_{j}\right\|_{6}^{6}+\varepsilon|\mathfrak{u}| \varepsilon^{-\frac{1}{2}}\left[\varepsilon^{\frac{1}{2}}\|w f\|_{\infty}\right]^{6} .
$$

Next, we need to bound $\int_{\gamma} \mathrm{d} \gamma \mathbf{1}_{\gamma_{+}}\left(1-P_{\gamma}^{\mathfrak{u}}\right) f \psi_{b}^{i, j}$. We have

$$
\left|\int_{\gamma} \mathrm{d} \gamma \mathbf{1}_{\gamma_{+}}\left(1-P_{\gamma}^{\mathfrak{u}}\right) f \psi_{b}^{i, j}\right| \leq\left\|\nabla_{x} \varphi_{b}^{j}\right\|_{L^{4 / 3}(\partial \Omega)}\left\|\mathbf{1}_{\gamma_{+}}\left(1-P_{\gamma}^{\mathfrak{u}}\right) f\right\|_{L^{4}(\gamma)} .
$$

Thus, we conclude that, for any $\eta>0$ and $\eta^{\prime}>0$

$$
\left.\left|\int_{\gamma} \mathrm{d} \gamma \mathbf{1}_{\gamma_{+}}\left(1-P_{\gamma}^{\mathfrak{u}}\right) f \psi_{b}^{i, j}\right| \lesssim \eta\left\|b_{j}\right\|_{6}^{6}+\eta^{\prime}\left[\varepsilon^{\frac{1}{2}}\|f\|_{\infty}\right]^{6}+C_{\eta, \eta^{\prime}}\left[\varepsilon^{-\frac{1}{2}}\left\|\mathbf{1}_{\gamma_{+}}\left(1-P_{\gamma}^{\mathfrak{u}}\right) f\right\|_{L^{2}(\gamma)}\right]^{6}\right\} .
$$

In conclusion, for any $\eta>0, \eta^{\prime}>0$, by

$$
\left.\left|\int_{\gamma} \mathrm{d} \gamma \psi_{b}^{i, j}\left[P_{\gamma}^{\mathfrak{u}} f+\mathbf{1}_{\gamma_{+}}\left(1-P_{\gamma}^{\mathfrak{u}}\right) f\right]\right| \lesssim \eta\left\|b_{j}\right\|_{6}^{6}+\eta^{\prime}\left[\varepsilon^{\frac{1}{2}}\|f\|_{\infty}\right]^{6}+\left.C_{\eta, \eta^{\prime}}\left[\varepsilon^{-\frac{1}{2}} \|\left(1-P_{\gamma}^{\mathfrak{u}}\right) f\right)\right|_{2 . \gamma_{+}}\right]^{6} .
$$

Finally,

$$
\left|\int_{\gamma_{-}} \mathrm{d} \gamma \varepsilon^{\frac{1}{2}} r \psi_{b}^{i, j}\right| \lesssim\left\|\nabla \varphi_{b}^{j}\right\|_{L^{4 / 3}(\partial \Omega)}\left\|\varepsilon^{\frac{1}{2}} r\right\|_{L^{4}(\partial \Omega)} \leq \varepsilon^{\frac{1}{2}}\|b\|_{6}^{5}|r|_{\infty} .
$$

By collecting all the terms and choosing $\eta$ and $\eta^{\prime}$ sufficiently small we conclude that

$$
\begin{aligned}
\left|\int_{\Omega^{c}} \mathrm{~d} x b_{i} \partial_{i} \partial_{j} \varphi_{b}^{j}\right| \lesssim\left(\varepsilon^{-1}\right. & \left.\left\|\left(\mathbf{I}-\mathbf{P}_{\mathfrak{u}}\right) f\right\|_{\nu}\right)^{6}+\left\|\left(\mathbf{I}-\mathbf{P}_{\mathfrak{u}}\right) f\right\|_{6}^{6}+\left\|g \nu^{-\frac{1}{2}}\right\|_{2}^{6}+\varepsilon|\mathfrak{u}|\left\|\mathbf{P}_{\mathfrak{u}} f\right\|_{6}^{6} \\
& +\eta\left\|b_{j}\right\|_{6}^{6}+\left(\varepsilon^{-\frac{1}{2}}\left|\left(1-P_{\gamma}^{\mathfrak{u}}\right) f\right|_{2,+}\right)^{6}+\left(\varepsilon^{\frac{1}{2}}|r|_{\infty}\right)^{6}+o(1)\left[\varepsilon^{\frac{1}{2}}\|f\|_{\infty}\right]^{6} .
\end{aligned}
$$

To estimate $\partial_{j}\left(\partial_{j} \Delta^{-1} b_{i}^{k-1}\right) b_{i}$ for $i \neq j$, we choose as test function

$$
\bar{\psi}_{b}^{i, j}=\left|v_{\mathfrak{u}}\right|^{2} v_{\mathfrak{u}, i} v_{\mathfrak{u}, j} \sqrt{\mu_{\mathfrak{u}}} \partial_{j} \varphi_{b}^{i}(x), \quad i \neq j .
$$

We have:

$$
\int_{\Omega^{c} \times \mathbb{R}^{3}} \mathrm{~d} x \mathrm{~d} v f v \cdot \nabla \bar{\psi}_{b}^{i, j}=\int_{\Omega^{c} \times \mathbb{R}^{3}} \mathrm{~d} x \mathrm{~d} v f v_{\mathfrak{u}} \cdot \nabla \bar{\psi}_{b}^{i, j}+\varepsilon \int_{\Omega^{c} \times \mathbb{R}^{3}} \mathrm{~d} x \mathrm{~d} v f \mathfrak{u} \cdot \nabla \bar{\psi}_{b}^{i, j} .
$$

By (3.20) and the Young inequality,

$$
\left|\varepsilon \int_{\Omega^{c} \times \mathbb{R}^{3}} \mathrm{~d} x \mathrm{~d} v f \mathfrak{u} \cdot \nabla \bar{\psi}_{b}^{i, j}\right| \leq \varepsilon|\mathfrak{u}|\left\|b_{j}\right\|_{6}^{5}\|f\|_{6} \leq \varepsilon|\mathfrak{u}|\left\|\mathbf{P}_{\mathfrak{u}} f\right\|_{6}^{6}+\varepsilon|\mathfrak{u}|\left\|\left(\mathbf{I}-\mathbf{P}_{\mathfrak{u}}\right) f\right\|_{6}^{6} .
$$

By using $f=\mathbf{P}_{\mathfrak{u}} f+\left(\mathbf{I}-\mathbf{P}_{\mathfrak{u}}\right) f$ and the expression of $\mathbf{P}_{\mathfrak{u}} f$, we need to compute

$$
\int_{\Omega^{c} \times \mathbb{R}^{3}} \mathrm{~d} x \mathrm{~d} v a \sqrt{\mu_{\mathfrak{u}}} v_{\mathfrak{u}} \cdot \nabla \bar{\psi}_{b}^{i, j}
$$




$$
\begin{gathered}
\int_{\Omega^{c} \times \mathbb{R}^{3}} \mathrm{~d} x \mathrm{~d} v b \cdot v_{\mathfrak{u}} \sqrt{\mu_{\mathfrak{u}}} v_{\mathfrak{u}} \cdot \nabla \bar{\psi}_{b}^{i, j} \\
\int_{\Omega^{c} \times \mathbb{R}^{3}} \mathrm{~d} x \mathrm{~d} v c \frac{\left|v_{\mathfrak{u}}\right|^{2}-3}{2} \sqrt{\mu_{\mathfrak{u}}} v_{\mathfrak{u}} \cdot \nabla \bar{\psi}_{b}^{i, j} \\
\int_{\Omega^{c} \times \mathbb{R}^{3}} \mathrm{~d} x \mathrm{~d} v v \cdot \nabla \bar{\psi}_{b}^{i, j}\left(\mathbf{I}-\mathbf{P}_{\mathfrak{u}}\right) f=\int_{\Omega^{c} \times \mathbb{R}^{3}} \mathrm{~d} x \mathrm{~d} v \sqrt{\mu_{\mathfrak{u}}}\left(\left|v_{\mathfrak{u}}\right|^{2}-\beta_{c}\right)^{2} v_{\mathfrak{u}} \otimes v_{\mathfrak{u}}: \nabla \otimes \nabla \varphi_{b}^{j}\left(\mathbf{I}-\mathbf{P}_{\mathfrak{u}}\right) f
\end{gathered}
$$

Using (3.20), the last one is bounded by

$$
\left\|\left(\mathbf{I}-\mathbf{P}_{\mathfrak{u}}\right)\right\|_{6}\left\|b_{j}\right\|_{L^{6}\left(\Omega^{c}\right)}^{5} \leq \frac{5}{6} \eta\left\|b_{j}\right\|_{L^{6}\left(\Omega^{c}\right)}^{6}+\frac{1}{6} \eta^{-\frac{1}{5}}\left\|\left(\mathbf{I}-\mathbf{P}_{\mathfrak{u}}\right)\right\|_{6}^{6},
$$

for any $\eta>0$.

For $j \neq i$, the $O(\mathfrak{u})$ terms in (3.30), (3.31) and (3.32) vanish by oddness in $v_{\mathfrak{u}, i}$. for the same reason the terms of order 1 in (3.30) and (3.32) vanish. The only surviving term is

$$
\sum_{k, \ell} b_{k} \partial_{\ell} \partial_{j} \varphi_{b}^{i} \int_{\Omega^{c} \times \mathbb{R}^{3}} \mathrm{~d} x \mathrm{~d} v \mu_{\mathfrak{u}} v_{\mathfrak{u}, k} v_{\mathfrak{u}, \ell} v_{\mathfrak{u}, i} v_{\mathfrak{u}, j}\left|v_{\mathfrak{u}}\right|^{2}=21 \int_{\Omega^{c}} \mathrm{~d} x\left(b_{j} \partial_{i} \partial_{j} \varphi_{b}^{i}+b_{i} \partial_{j}^{2} \varphi_{b}^{i}\right),
$$

because

$$
\int_{\mathbb{R}^{3}} \mathrm{~d} v \mu_{\mathfrak{u}} v_{\mathfrak{u}, k} v_{\mathfrak{u}, \ell} v_{\mathfrak{u}, i} v_{\mathfrak{u}, j}\left|v_{\mathfrak{u}}\right|^{2}=21\left(\delta_{k, \ell} \delta_{i, j}+\delta_{k, i} \delta_{\ell, j}+\delta_{k, j} \delta_{\ell, i}\right)
$$

By taking the sum on $j$ this reduces to $\int_{\Omega^{c}} d x\left(b_{i}^{6}+\sum_{j} b_{j} \partial_{j} \partial_{i} \Delta^{-1} b_{i}\right)$. The second term has been bounded in (3.28), thus, to complete the estimate of $\|b\|_{6}$ we just need to bound the remaining terms in the weak equation (3.1) for $\psi=\bar{\psi}_{b}^{i, j}$. As before, we have

$\varepsilon^{-1}\left|\int_{\Omega^{c} \times \mathbb{R}^{3}} \mathrm{~d} x \mathrm{~d} v \bar{\psi}_{b}^{i, j} L_{\mathfrak{u}} f\right| \leq \varepsilon^{-1}\left\|\nabla \varphi_{b}^{j}\right\|_{L^{2}\left(\Omega^{c}\right)}\left\|\left(\mathbf{I}-\mathbf{P}_{\mathfrak{u}}\right) f\right\|_{\nu} \leq \frac{5}{6} \eta\left\|b_{j}\right\|_{6}^{6}+\frac{1}{6} \eta^{-\frac{1}{5}}\left[\varepsilon^{-1}\left\|\left(\mathbf{I}-\mathbf{P}_{\mathfrak{u}}\right) f\right\|_{\nu}\right]^{6}$, and

$$
\left|\int_{\Omega^{c} \times \mathbb{R}^{3}} \mathrm{~d} x \mathrm{~d} v \bar{\psi}_{b}^{i, j} g\right| \lesssim\left\|\nabla \varphi_{b_{j}}\right\|_{L^{2}\left(\Omega^{c}\right)}\left\|g \nu^{-\frac{1}{2}}\right\|_{2} \leq \frac{5}{6} \eta\left\|b_{j}\right\|_{6}^{6}+\frac{1}{6} \eta^{-\frac{1}{5}}\left\|g \nu^{-\frac{1}{2}}\right\|_{2},
$$

for any $\eta>0$. Finally, expanding, we have

$$
\begin{aligned}
& \left|v_{\mathfrak{u}}\right|^{2} v_{\mathfrak{u}, i} v_{\mathfrak{u}, j}=|v|^{2} v_{i} v_{j}+\varepsilon\left[|v|^{2}\left(\mathfrak{u}_{i} v_{j}+\mathfrak{u}_{j} v_{i}\right)-2 \mathfrak{u} \cdot v v_{i} v_{i}\right] \\
& \quad+\varepsilon^{2}\left[\left.|\mathfrak{u}|^{2} v_{i} v_{j}|| v\right|^{2} \mathfrak{u}_{i} \mathfrak{u}_{j}-2 \mathfrak{u} \cdot v\left(\mathfrak{u}_{i} v_{j}+\mathfrak{u}_{j} v_{i}\right)\right]+\varepsilon^{3}\left[|\mathfrak{u}|^{2}\left(\mathfrak{u}_{j} v_{j}+\mathfrak{u}_{j} v_{i}-2 \mathfrak{u} \cdot v \mathfrak{u}_{i} \mathfrak{u}_{j}\right]+\varepsilon^{4}|\mathfrak{u}|^{2} \mathfrak{u}_{i} \mathfrak{u}_{j} .\right.
\end{aligned}
$$

Therefore in the contribution from $P_{\gamma}^{\mathfrak{u}} f$ the term of order 0 in $\varepsilon$ gives a vanishing contribution. Therefore, as before

$$
\left|\int_{\gamma} \mathrm{d} \gamma P_{\gamma}^{\mathfrak{u}} f \bar{\psi}_{b}^{i, j}\right| \leq \varepsilon|\mathfrak{u}| \varepsilon^{-\frac{1}{2}}\left[\varepsilon^{\frac{1}{2}}\|f\|_{\infty}\right]\left\|b_{j}\right\|_{6}^{5} \lesssim \varepsilon^{\frac{1}{2}}|\mathfrak{u}|\left\|b_{j}\right\|_{6}^{6}+\varepsilon|\mathfrak{u}| \varepsilon^{-\frac{1}{2}}\left[\varepsilon^{\frac{1}{2}}\|w f\|_{\infty}\right]^{6} .
$$

Moreover

$$
\left|\int_{\gamma} \mathrm{d} \gamma \mathbf{1}_{\gamma_{+}}\left(1-P_{\gamma}^{\mathfrak{u}}\right) f \bar{\psi}_{b}^{i, j}\right| \leq\left\|\nabla_{x} \varphi_{b}^{j}\right\|_{L^{4 / 3}(\partial \Omega)}\left\|\mathbf{1}_{\gamma_{+}}\left(1-P_{\gamma}^{\mathfrak{u}}\right) f\right\|_{L^{4}(\gamma)} .
$$

By collecting the previous bounds we conclude that

$$
\begin{aligned}
\|b\|_{6}^{6} \lesssim\left(\varepsilon^{-1}\left\|\left(\mathbf{I}-\mathbf{P}_{\mathfrak{u}}\right) f\right\|_{\nu}\right)^{6}+\left\|\left(\mathbf{I}-\mathbf{P}_{\mathfrak{u}}\right) f\right\|_{6}^{6} & +\left\|g \nu^{-\frac{1}{2}}\right\|_{2}^{6}+\left(\varepsilon^{-\frac{1}{2}}\left|\left(1-P_{\gamma}^{\mathfrak{u}}\right) f\right|_{2,+}\right)^{6} \\
& +\varepsilon|\mathfrak{u}|\left\|\mathbf{P}_{\mathfrak{u}} f\right\|_{6}+\left(\varepsilon^{\frac{1}{2}}|r|_{\infty}\right)^{6}+o(1)\left[\varepsilon^{\frac{1}{2}} f \|_{\infty}\right]^{6} .
\end{aligned}
$$

Step 3:

Then we bound $\|a\|_{6}$. The argument is similar to the one used for $c$, the only main difference being in the treatment of the boundary terms.

$$
\psi=\psi_{a} \equiv\left(\left|v_{\mathfrak{u}}\right|^{2}-\beta_{a}\right) v_{\mathfrak{u}} \cdot \nabla_{x} \varphi_{a} \sqrt{\mu}=\sum_{i=1}^{d}\left(\left|v_{\mathfrak{u}}\right|^{2}-\beta_{a}\right) v_{\mathfrak{u}, i} \partial_{i} \varphi_{a} \sqrt{\mu},
$$

where

$$
-\Delta_{x} \varphi_{a}(x)=a^{5},\left.\quad \frac{\partial}{\partial n} \varphi_{a}\right|_{\partial \Omega}=0
$$


whose solution satisfies

$$
\left\|\nabla \varphi_{a}\right\|_{\dot{H}^{1}\left(\Omega^{c}\right)} \leq\left\||a|^{5}\right\|_{L^{\frac{6}{5}\left(\Omega^{c}\right)}}=\|a\|_{L^{6}\left(\Omega^{c}\right)}^{5}
$$

We have

$$
\int_{\Omega^{c} \times \mathbb{R}^{3}} \mathrm{~d} x \mathrm{~d} v f v \cdot \nabla \psi_{a}=\int_{\Omega^{c} \times \mathbb{R}^{3}} \mathrm{~d} x \mathrm{~d} v f(v-\varepsilon \mathfrak{u}) \cdot \nabla \psi_{a}+\varepsilon \int_{\Omega^{c} \times \mathbb{R}^{3}} \mathrm{~d} x \mathrm{~d} v f \mathfrak{u} \cdot \nabla \psi_{a} .
$$

By (3.38) and the Young inequality,

$$
\left|\varepsilon \int_{\Omega^{c} \times \mathbb{R}^{3}} \mathrm{~d} x \mathrm{~d} v f \mathfrak{u} \cdot \nabla \psi_{a}\right| \leq \varepsilon|\mathfrak{u}|\left\|b_{j}\right\|_{6}^{5}\|f\|_{6} \leq \varepsilon|\mathfrak{u}|\left\|\mathbf{P}_{\mathfrak{u}} f\right\|_{6}^{6}+\varepsilon|\mathfrak{u}|\left\|\left(\mathbf{I}-\mathbf{P}_{\mathfrak{u}}\right) f\right\|_{6}^{6} .
$$

Proceeding as before, by using $f=\mathbf{P}_{\mathfrak{u}} f+\left(\mathbf{I}-\mathbf{P}_{\mathfrak{u}}\right) f$ and the expression of $\mathbf{P}_{\mathfrak{u}} f$, we need to compute

$$
\begin{gathered}
\int_{\Omega^{c} \times \mathbb{R}^{3}} \mathrm{~d} x \mathrm{~d} v a \sqrt{\mu_{\mathfrak{u}}} v_{\mathfrak{u}} \cdot \nabla \psi_{a}, \\
\int_{\Omega^{c} \times \mathbb{R}^{3}} \mathrm{~d} x \mathrm{~d} v b \cdot v_{\mathfrak{u}} \sqrt{\mu_{\mathfrak{u}}} v_{\mathfrak{u}} \cdot \nabla \psi_{a}, \\
\int_{\Omega^{c} \times \mathbb{R}^{3}} \mathrm{~d} x \mathrm{~d} v c \frac{\left|v_{\mathfrak{u}}\right|^{2}-3}{2} \sqrt{\mu_{\mathfrak{u}}} v_{\mathfrak{u}} \cdot \nabla \psi_{a}, \\
\int_{\Omega^{c} \times \mathbb{R}^{3}} \mathrm{~d} x \mathrm{~d} v v \cdot \nabla \psi_{a}\left(\mathbf{I}-\mathbf{P}_{\mathfrak{u}}\right) f=\int_{\Omega \times \mathbb{R}^{3}} \mathrm{~d} x \mathrm{~d} v \sqrt{\mu_{\mathfrak{u}}}\left(\left|v_{\mathfrak{u}}\right|^{2}-\beta_{a}\right)^{2} v_{\mathfrak{u}} \otimes v_{\mathfrak{u}}: \nabla \otimes \nabla \varphi_{a}\left(\mathbf{I}-\mathbf{P}_{\mathfrak{u}}\right) f .
\end{gathered}
$$

Using (3.38), by the Young inequality, the last one is bounded by

$$
\left\|\left(\mathbf{I}-\mathbf{P}_{\mathfrak{u}}\right) f\right\|_{6}\|c\|_{L^{6}\left(\Omega^{c}\right)}^{5} \leq \frac{5}{6} \eta\|a\|_{L^{6}\left(\Omega^{c}\right)}^{6}+\frac{1}{6} \eta^{-\frac{1}{5}}\left\|\left(\mathbf{I}-\mathbf{P}_{\mathfrak{u}}\right)\right\|_{6}^{6}
$$

for any $\eta>0$.

With the choice $\beta_{a}=10$

$$
\int_{\mathbb{R}^{3}} \mathrm{~d} v\left(\left|v_{\mathfrak{u}}\right|^{2}-\beta_{a}\right)\left(\left|v_{\mathfrak{u}}\right|^{2}-3\right) v_{\mathfrak{u}} \otimes v_{\mathfrak{u}}=0,
$$

and the term in (3.41) vanishes. The term of (3.40) vanishes for the same reason.

Now we compute the term in (3.39): we have

$$
\begin{array}{r}
\int_{\Omega^{c} \times \mathbb{R}^{3}} \mathrm{~d} x \mathrm{~d} v a \sqrt{\mu_{\mathfrak{u}}} v_{\mathfrak{u}} \cdot \nabla \psi_{a}=\int_{\Omega^{c}} \mathrm{~d} x a \nabla \otimes \nabla \varphi_{a}: \int_{\mathbb{R}^{3}} \mathrm{~d} v v_{\mathfrak{u}} \otimes v_{\mathfrak{u}} v_{\mathfrak{u}}\left(\left|v_{\mathfrak{u}}\right|^{2}-\beta_{a}\right) \mu_{\mathfrak{u}} \\
=-5 \int_{\Omega^{c}} \mathrm{~d} x a \Delta \varphi_{a}=5\|a\|_{L^{6}\left(\Omega^{c}\right)}^{6},
\end{array}
$$

because of (3.37). We have used

$$
\int_{\mathbb{R}^{3}} \mathrm{~d} x \mathrm{~d} v v_{\mathfrak{u}} \otimes v_{\mathfrak{u}}\left(\left|v_{\mathfrak{u}}\right|^{2}-\beta_{a}\right) \mu_{\mathfrak{u}}=-5 \mathbf{I} .
$$

As for the boundary term, we have

$$
\int_{\gamma} \mathrm{d} \gamma P_{\gamma}^{\mathfrak{u}} f \psi_{a}=\int_{\partial \Omega} \mathrm{d} S z_{\gamma} \nabla \varphi_{a} \cdot \int_{\mathbb{R}^{3}} \mathrm{~d} v \mu(v-\varepsilon \mathfrak{u})\left(|v-\varepsilon \mathfrak{u}|^{2}-\beta_{a}\right) n \cdot v
$$

But

$$
\int_{\mathbb{R}^{3}} \mathrm{~d} v \mu v_{\mathfrak{u}}\left(\left|v_{\mathfrak{u}}\right|^{2}-\beta_{a}\right) n \cdot v=\int_{\mathbb{R}^{3}} \mathrm{~d} v \mu v_{\mathfrak{u}}\left(\left|v_{\mathfrak{u}}\right|^{2}-\beta_{a}\right) n \cdot v_{\mathfrak{u}}+\varepsilon \int_{\mathbb{R}^{3}} \mathrm{~d} v \mu v_{\mathfrak{u}}\left(\left|v_{\mathfrak{u}}\right|^{2}-\beta_{a}\right) n \cdot \mathfrak{u} .
$$

The second term vanishes by oddness. The first by oddness is

$$
\int_{\mathbb{R}^{3}} d v \mu v_{\mathfrak{u}, i}\left(\left|v_{\mathfrak{u}}\right|^{2}-\beta_{a}\right) n \cdot v_{\mathfrak{u}}=n_{i} \int_{\mathbb{R}^{3}} \mathrm{~d} v \mu\left|v_{\mathfrak{u}} \cdot n\right|^{2}\left(\left|v_{\mathfrak{u}}\right|^{2}-\beta_{a}\right)=-5 n_{i} .
$$

Therefore

$$
\int_{\gamma} \mathrm{d} \gamma P_{\gamma}^{\mathfrak{u}} f \psi_{a}=\int_{\partial \Omega} d S z_{\gamma} n \cdot \nabla \varphi_{a}=0
$$


by the Neumann boundary condition on $\varphi_{a}$. The term $\int_{\gamma} \mathrm{d} \gamma \mathbf{1}_{\gamma_{+}}\left(1-P_{\gamma}^{\mathfrak{u}}\right) f \psi_{a}$ is estimated as the similar term for $c$. By collecting the estimate, we conclude that

$$
\begin{array}{r}
\|a\|_{6} \lesssim \varepsilon^{-1}\left\|\left(\mathbf{I}-\mathbf{P}_{\mathfrak{u}}\right) f\right\|_{\nu}+\left\|\left(\mathbf{I}-\mathbf{P}_{\mathfrak{u}}\right) f\right\|_{6}+\left\|g \nu^{-\frac{1}{2}}\right\|_{2}+(\varepsilon|\mathfrak{u}|)^{\frac{1}{6}}\left\|\mathbf{P}_{\mathfrak{u}} f\right\|_{6}+\varepsilon^{-\frac{1}{2}}\left|\left(1-P_{\gamma}^{\mathfrak{u}}\right) f\right|_{2,+} \\
+\varepsilon^{\frac{1}{2}}|r|_{\infty}+o(1)\left[\varepsilon^{\frac{1}{2}}\|f\|_{\infty}\right] .
\end{array}
$$

In conclusion, for $|\mathfrak{u}|$ small,

$\left\|\mathbf{P}_{\mathfrak{u}} f\right\|_{6} \lesssim \varepsilon^{-1}\left\|\left(\mathbf{I}-\mathbf{P}_{\mathfrak{u}}\right) f\right\|_{\nu}+\left\|\left(\mathbf{I}-\mathbf{P}_{\mathfrak{u}}\right)\right\|_{6}+\left\|g \nu^{-\frac{1}{2}}\right\|_{2}+\varepsilon^{-\frac{1}{2}}\left|\left(1-P_{\gamma}^{\mathfrak{u}}\right) f\right|_{2,+}+\varepsilon^{\frac{1}{2}}|r|_{\infty}+o(1)\left[\varepsilon^{\frac{1}{2}}\|f\|_{\infty}\right]$.

\section{Balance LaWS}

The mass, momentum and energy balance equations are obtained by projecting (1.42) on the null space of $L_{\mathfrak{u}}$. Since $\mathbf{P}_{\mathfrak{u}} L_{\mathfrak{u}}=0$, we have:

$$
\mathbf{P}_{\mathfrak{u}}(v \cdot \nabla f)=\mathbf{P}_{\mathfrak{u}} g .
$$

More explicitly, we write $\mathbf{P}_{\mathfrak{u}} g=\left(\mathfrak{a}+\mathfrak{b} \cdot v_{\mathfrak{u}}+\frac{1}{2}\left(\left|v_{\mathfrak{u}}\right|^{2}-3\right) \mathfrak{c}\right) \sqrt{\mu}_{\mathfrak{u}}$, and $\mathbf{P}_{\mathfrak{u}} f=\left[a+b \cdot v_{\mathfrak{u}}+\frac{1}{2}\left(\left|v_{\mathfrak{u}}\right|^{2}-\right.\right.$ 3)c] $\sqrt{\mu}_{\mathfrak{u}}$. We have

$$
\begin{aligned}
& \nabla \cdot b+\varepsilon \mathfrak{u} \cdot \nabla a=\mathfrak{a}, \\
& \nabla P+\varepsilon \mathfrak{u} \cdot \nabla b+\nabla \cdot \tau=\mathfrak{b}, \\
& \nabla \cdot b+\frac{3}{2} \varepsilon \mathfrak{u} \cdot \nabla c+\nabla \cdot \mathfrak{q}=\mathfrak{c},
\end{aligned}
$$

where

$$
\begin{gathered}
\tau=\int_{\mathbb{R}^{3}} \mathrm{~d} v v_{\mathfrak{u}} \otimes v_{\mathfrak{u}} \sqrt{\mu_{\mathfrak{u}}}\left(\mathbf{I}-\mathbf{P}_{\mathfrak{u}}\right) f, \\
\mathfrak{q}=\int_{\mathbb{R}^{3}} \mathrm{~d} v \frac{\left|v_{\mathfrak{u}}\right|^{2}-3}{2} v_{\mathfrak{u}} \sqrt{\mu_{\mathfrak{u}}}\left(\mathbf{I}-\mathbf{P}_{\mathfrak{u}}\right) f, \\
P=a+c .
\end{gathered}
$$

We have to complete equations (4.2), (4.3), (4.4) with boundary conditions following from (1.42), which are not immediately translated into conditions on $a, b, c$. Therefore, as in [24], we introduce a smooth cutoff function

$$
\zeta(x)= \begin{cases}1 & \text { if } x \in \mathbb{R}^{3} \backslash \Omega \text { and } d(x, \Omega)>1 \\ 0 & \text { if } x \in \bar{\Omega}\end{cases}
$$

and define $f^{\zeta}=\zeta f$ extended as 0 in $\Omega$. If $f$ solves the problem (1.42), then $f^{\zeta}$ solves the equation

$$
v \cdot \nabla f^{\zeta}+\varepsilon^{-1} L_{\mathfrak{u}} f^{\zeta}=\zeta g+\mathcal{C} \quad \text { in } \quad \mathbb{R}^{3},
$$

where

$$
\mathcal{C}=f v \cdot \nabla \zeta
$$

By projecting the equation for $f^{\zeta}$ on the null space of $L_{\mathfrak{u}}$ we obtain the balance laws

$$
\mathbf{P}_{\mathfrak{u}}\left(v \cdot \nabla f^{\zeta}\right)=\mathbf{P}_{\mathfrak{u}} \mathcal{C}+\zeta \mathbf{P}_{\mathfrak{u}} g,
$$

More explicitly, with $\mathbf{P}_{\mathfrak{u}} f^{\zeta}=\left[a^{\zeta}+b^{\zeta} \cdot v_{\mathfrak{u}}+c^{\zeta}\left(\left|v_{\mathfrak{u}}\right|^{2}-3\right) / 2\right] \sqrt{\mu_{\mathfrak{u}}}$ and $P^{\zeta}=a^{\zeta}+c^{\zeta}$, we have,

$$
\begin{aligned}
& \nabla \cdot b^{\zeta}+\varepsilon \mathfrak{u} \cdot \nabla a^{\zeta}=\zeta \mathfrak{a}+\int_{\mathbb{R}^{3}} \mathrm{~d} v \mathcal{C}, \\
& \nabla P^{\zeta}+\varepsilon \mathfrak{u} \cdot \nabla b^{\zeta}+\nabla \cdot \tau^{\zeta}=\zeta \mathfrak{b}+\int_{\mathbb{R}^{3}} \mathrm{~d} v \mathcal{C} v \sqrt{\mu}_{\mathfrak{u}} \\
& \nabla \cdot b^{\zeta}+\frac{3}{2} \varepsilon \mathfrak{u} \cdot \nabla c^{\zeta}+\nabla \cdot q^{\zeta}=\zeta \mathfrak{c}+\int_{\mathbb{R}^{3}} \mathrm{~d} v \frac{1}{2} \mathcal{C}\left(|v|^{2}-3\right) \sqrt{\mu}
\end{aligned}
$$

where

$$
\tau^{\zeta}=\int_{\mathbb{R}^{3}} \mathrm{~d} v v_{\mathfrak{u}} \otimes v_{\mathfrak{u}} \sqrt{\mu_{\mathfrak{u}}}\left(\mathbf{I}-\mathbf{P}_{\mathfrak{u}}\right) f^{\zeta}
$$




$$
q^{\zeta}=\int_{\mathbb{R}^{3}} \mathrm{~d} v \frac{\left|v_{\mathfrak{u}}\right|^{2}-3}{2} v_{\mathfrak{u}} \sqrt{\mu_{\mathfrak{u}}}\left(\mathbf{I}-\mathbf{P}_{\mathfrak{u}}\right) f^{\zeta}
$$

and $P^{\zeta}=a^{\zeta}+c^{\zeta}$.

It is convenient to write above equations in the Fourier space: The Fourier transform is normalized as

$$
\hat{f}(k)=\mathcal{F}_{x}(f)(k)=\frac{1}{(2 \pi)^{\frac{3}{2}}} \int_{\mathbb{R}^{3}} \mathrm{~d} x f(x) e^{i k \cdot x} .
$$

We have

$$
i k \cdot v \hat{f}^{\zeta}+\varepsilon^{-1} L_{\mathfrak{u}} f^{\zeta}=\widehat{\zeta g}+\hat{\mathcal{C}}
$$

By writing

$$
\hat{f}^{\zeta}=\left(\hat{a}^{\zeta}+\hat{b}^{\zeta} \cdot v_{\mathfrak{u}}+\frac{1}{2} \hat{c}^{\zeta}\left(\left|v_{\mathfrak{u}}\right|^{2}-3\right)\right) \sqrt{\mu}_{\mathfrak{u}}+\left(\mathbf{I}-\mathbf{P}_{\mathfrak{u}}\right) \hat{f}^{\zeta}
$$

the projection on $\mathrm{Null}_{\mathfrak{u}}$ is

$$
\begin{aligned}
& i k \cdot \hat{b}^{\zeta}+i \varepsilon k \cdot \mathfrak{u} \hat{a}^{\zeta}=\int_{\mathbb{R}^{3}} \mathrm{~d} v \sqrt{\mu}_{\mathfrak{u}} \hat{\mathcal{C}}(k, v)+\widehat{\zeta \mathfrak{a}}, \\
& i k \hat{P}^{\zeta}+i k \cdot \hat{\tau}^{\zeta}+i \varepsilon \mathfrak{u} \cdot k \hat{b}^{\zeta}=\int_{\mathbb{R}^{3}} \mathrm{~d} v v_{\mathfrak{u}} \sqrt{\mu}_{\mathfrak{u}} \hat{\mathcal{C}}(k, v)+\widehat{\zeta \mathfrak{b}} \\
& i k \cdot \hat{b}^{\zeta}+\frac{3}{2} i \varepsilon k \cdot \mathfrak{u} \hat{c}^{\zeta}+i k \cdot \mathfrak{q}^{\zeta}=\int_{\mathbb{R}^{3}} \mathrm{~d} v \frac{1}{2}\left(\left|v_{\mathfrak{u}}\right|^{2}-3\right) \sqrt{\mu} \hat{\mathcal{C}}(k, v)+\widehat{\zeta} \mathfrak{c} .
\end{aligned}
$$

Let

$$
\mathscr{B}_{\mathfrak{u}}=L_{\mathfrak{u}}^{-1}\left[\left(v_{\mathfrak{u}} \otimes v_{\mathfrak{u}}-\frac{1}{3}\left|v_{\mathfrak{u}}\right|^{2} \mathbf{I}\right) \sqrt{\mu_{\mathfrak{u}}}\right], \quad \mathscr{A}_{\mathfrak{u}}=L_{\mathfrak{u}}^{-1}\left[\frac{1}{2}\left(v_{\mathfrak{u}}\left(\left|v_{\mathfrak{u}}\right|^{2}-5\right) \sqrt{\mu}_{\mathfrak{u}}\right)\right]
$$

The momentum equation (4.19) then becomes

$$
i k \hat{P}^{\zeta}+i \varepsilon \mathfrak{u} \cdot k \hat{b}^{\zeta}+i k \cdot \int_{\mathbb{R}^{3}} \mathrm{~d} v L_{\mathfrak{u}} \hat{f}^{\zeta} \mathscr{B}_{\mathfrak{u}}=\int_{\mathbb{R}^{3}} \mathrm{~d} v v_{\mathfrak{u}} \sqrt{\mu}_{\mathfrak{u}} \hat{\mathcal{C}}(k, v)+\widehat{\zeta \mathfrak{b}},
$$

and the energy equation (4.20) becomes

$$
i k \cdot \hat{b}^{\zeta}+\frac{3}{2} i \varepsilon k \cdot \mathfrak{u} \hat{c}^{\zeta}+i k \cdot \int_{\mathbb{R}^{3}} \mathrm{~d} v L_{\mathfrak{u}} \hat{f}^{\zeta} \mathscr{A}_{\mathfrak{u}}=\int_{\mathbb{R}^{3}} \mathrm{~d} v \frac{1}{2}\left(\left|v_{\mathfrak{u}}\right|^{2}-3\right) \sqrt{\mu_{\mathfrak{u}}} \hat{\mathcal{C}}(k, v)+\widehat{\zeta \mathfrak{c}}
$$

Substituting from the equation (4.16),$L_{\mathfrak{u}} \hat{f}^{\zeta}=-\varepsilon i k \cdot v \hat{f} \zeta+\varepsilon(\widehat{\zeta g}+\hat{\mathcal{C}})$,

$$
\begin{aligned}
& i k\left(\hat{a}^{\zeta}+\hat{c}^{\zeta}\right)+i \varepsilon \mathfrak{u} \cdot k \hat{b}^{\zeta}+i k \cdot \int_{\mathbb{R}^{3}} \mathrm{~d} v\left[-i \varepsilon k \cdot v \hat{f}^{\zeta}+\varepsilon(\widehat{\zeta g}+\hat{\mathcal{C}})\right] \mathscr{B}_{\mathfrak{u}}=\widehat{\zeta \mathfrak{b}}+\int_{\mathbb{R}^{3}} \mathrm{~d} v v_{\mathfrak{u}} \sqrt{\mu}_{\mathfrak{u}} \hat{\mathcal{C}}(k, v) \\
& i k \cdot \hat{b}^{\zeta}+i \varepsilon \frac{3}{2} k \cdot \mathfrak{u} \hat{c}^{\zeta}+i k \cdot \int_{\mathbb{R}^{3}} \mathrm{~d} v\left[-i \varepsilon k \cdot v \hat{f} \zeta+\varepsilon(\widehat{\zeta g}+\hat{\mathcal{C}}) \mathscr{A}_{\mathfrak{u}}=\widehat{\zeta \mathfrak{c}}+\int_{\mathbb{R}^{3}} \mathrm{~d} v \frac{1}{2}\left(\left|v_{\mathfrak{u}}\right|^{2}-3\right) \sqrt{\mu}_{\mathfrak{u}} \hat{\mathcal{C}}(k, v) .\right.
\end{aligned}
$$

Using again (4.17), the term $\int d v \hat{f} \zeta v \cdot \mathscr{B}_{\mathfrak{u}}$ becomes

$$
\begin{array}{r}
\int_{\mathbb{R}^{3}} \mathrm{~d} v \hat{f}^{\zeta} v \cdot \mathscr{B}_{\mathfrak{u}}=\int_{\mathbb{R}^{3}} \mathrm{~d} v v_{\mathfrak{u}} \cdot\left(\hat{a}^{\zeta}+\hat{b}^{\zeta} \cdot v_{\mathfrak{u}}+\hat{c}^{\zeta}\left(\left|v_{\mathfrak{u}}\right|^{2}-3\right) / 2\right) \sqrt{\mu_{\mathfrak{u}}} \mathscr{B}_{\mathfrak{u}}+\int_{\mathbb{R}^{3}} \mathrm{~d} v v \cdot \mathscr{B}_{\mathfrak{u}}\left(\mathbf{I}-\mathbf{P}_{\mathfrak{u}}\right) \hat{f}^{\zeta} \\
+\varepsilon \int_{\mathbb{R}^{3}} \mathrm{~d} v \mathfrak{u} \cdot \mathscr{B}_{\mathfrak{u}}\left(\hat{a}^{\zeta}+\hat{b} \zeta v_{\mathfrak{u}}+\frac{1}{2} \hat{c}^{\zeta}\left(\left|v_{\mathfrak{u}}\right|^{2}-3\right)\right) \sqrt{\mu}_{\mathfrak{u}}
\end{array}
$$

The second line vanishes because $\mathbf{P}_{\mathfrak{u}} \mathscr{B}_{\mathfrak{u}}=0$. From the properties of $\mathscr{B}_{\mathfrak{u}}$, only the $\hat{b}$ term survives of the first part of first line. Since, again $\left(\mathbf{I}-\mathbf{P}_{\mathfrak{u}}\right)\left(v_{\mathfrak{u}} \otimes v_{\mathfrak{u}}\right) \sqrt{\mu}_{\mathfrak{u}}=L_{\mathfrak{u}} \mathscr{B}_{\mathfrak{u}}$, we obtain

$$
\int_{\mathbb{R}^{3}} \mathrm{~d} v \hat{f}^{\zeta} v \cdot \mathscr{B}_{\mathfrak{u}}=\hat{b}^{\zeta} \int_{\mathbb{R}^{3}} \mathrm{~d} v \mathscr{B}_{\mathfrak{u}} L_{\mathfrak{u}} \mathscr{B}_{\mathfrak{u}}+\int_{\mathbb{R}^{3}} \mathrm{~d} v v \cdot \mathscr{B}_{\mathfrak{u}}\left(\mathbf{I}-\mathbf{P}_{\mathfrak{u}}\right) \hat{f}^{\zeta}
$$

We have (see e.g. [6])

$$
-\int d v L_{u} \mathscr{B}_{\mathfrak{u}} \otimes \mathscr{B}_{\mathfrak{u}} \cdot k k \cdot \hat{b}{ }^{\zeta} \sqrt{\mu}=\mathbb{K} b^{\zeta}
$$

with

$$
\mathbb{K}=\mathfrak{v}\left[|k|^{2} \mathbf{I}+\frac{2}{3} k \otimes k\right]
$$


with $\mathfrak{v}>0$ the viscosity coefficient. Note that for any $y \in \mathbb{R}^{3}$ with $|y|=1$,

$$
y \cdot \mathbb{K} y \sim \mathfrak{v}|k|^{2}, \quad y \cdot \mathbb{K}^{-1} y \sim \mathfrak{v}^{-1}|k|^{-2}
$$

We obtain:

$$
\begin{aligned}
i k\left(\hat{a}^{\zeta}+\hat{c}^{\zeta}\right)+i \varepsilon \mathfrak{u} \cdot k \hat{b}^{\zeta}+\varepsilon \mathbb{K} b^{\zeta}+\varepsilon k \otimes k \cdot \int_{\mathbb{R}^{3}} \mathrm{~d} v v \mathscr{B}_{\mathfrak{u}}(\mathbf{I}-\mathbf{P}) \hat{f}^{\zeta}+\varepsilon i k \cdot \int(\widehat{\zeta g}+\hat{\mathcal{C}}) \mathscr{B}_{\mathfrak{u}} \\
=\widehat{\zeta \mathfrak{b}}+\int_{\mathbb{R}^{3}} \mathrm{~d} v v_{\mathfrak{u}} \sqrt{\mu}_{\mathfrak{u}} \hat{\mathcal{C}}(k, v)
\end{aligned}
$$

Similarly, since $\left(\mathbf{I}-\mathbf{P}_{\mathfrak{u}}\right)\left[v_{\mathfrak{u}}\left(\left|v_{\mathfrak{u}}\right|^{2}-3\right) / 2 \sqrt{\mu}_{\mathfrak{u}}\right]=L_{\mathfrak{u}} \mathscr{A}_{\mathfrak{u}}$,

$$
\begin{aligned}
i k \cdot \hat{b}^{\zeta}+i \varepsilon \frac{3}{2} k \cdot \mathfrak{u} \hat{c}^{\zeta}+\varepsilon \kappa|k|^{2} \hat{c}^{\zeta}+\varepsilon k \otimes k \cdot \int_{\mathbb{R}^{3}} \mathrm{~d} v v\left(\mathbf{I}-\mathbf{P}_{\mathfrak{u}}\right) \hat{f}^{\zeta} \mathscr{A}_{\mathfrak{u}}+\varepsilon i k \cdot \int_{\mathbb{R}^{3}} \mathrm{~d} v(\widehat{\zeta g}+\hat{\mathcal{C}}) \mathscr{A}_{\mathfrak{u}} \\
=\widehat{\zeta \mathfrak{c}}+\int_{\mathbb{R}^{3}} \mathrm{~d} v \frac{1}{2}\left(\left|v_{\mathfrak{u}}\right|^{2}-3\right) \sqrt{\mu}_{\mathfrak{u}} \hat{\mathcal{C}}(k, v),
\end{aligned}
$$

with $\kappa=\int_{\mathbb{R}^{3}} \mathrm{~d} v \mathscr{A} L \mathscr{A}$. Therefore the balance laws in the Fourier space are

$$
\begin{aligned}
& i k \cdot \hat{b}^{\zeta}+i \varepsilon k \cdot \mathfrak{u} \hat{a}^{\zeta}=\hat{s}_{0}, \\
& i k \hat{P}^{\zeta}+\varepsilon \mathbb{K} b^{\zeta}+i \varepsilon \mathfrak{u} \cdot k \hat{b}^{\zeta}=\underline{\hat{s}} \\
& \left.i k \cdot \hat{b}^{\zeta}+\varepsilon \kappa|k|^{2}+\frac{3}{2} i k \cdot \mathfrak{u}\right] \hat{c}^{\zeta}=\hat{s}_{4},
\end{aligned}
$$

where the transport coefficient $\kappa$ is defined by $\kappa=\int d v \mathscr{A}_{\mathfrak{u}} L_{\mathfrak{u}}^{-1} \mathscr{A}_{\mathfrak{u}}$ and the source terms are

$$
\begin{aligned}
\hat{s}_{0} & =\int_{\mathbb{R}^{3}} \mathrm{~d} v \sqrt{\mu} \hat{\mathcal{C}}(k, v)+\widehat{\zeta \mathfrak{a}} \\
\underline{\hat{s}} & =-\varepsilon k \otimes k \cdot \int_{\mathbb{R}^{3}} \mathrm{~d} v v\left(\mathbf{I}-\mathbf{P}_{\mathfrak{u}}\right) \hat{f} \zeta \mathscr{B}_{\mathfrak{u}}-i \varepsilon k \cdot \int_{\mathbb{R}^{3}} \mathrm{~d} v(\widehat{\zeta g}+\hat{\mathcal{C}}) \mathscr{B}_{\mathfrak{u}}+\int_{\mathbb{R}^{3}} \mathrm{~d} v v_{\mathfrak{u}} \sqrt{\mu} \hat{\mathcal{C}}(k, v) \\
& +\widehat{\zeta \mathfrak{b}} \\
\hat{s}_{4} & =-\varepsilon k \otimes k \cdot \int_{\mathbb{R}^{3}} \mathrm{~d} v v\left(\mathbf{I}-\mathbf{P}_{\mathfrak{u}}\right) \hat{f}^{\zeta} \mathscr{A}_{\mathfrak{u}}-i \varepsilon k \cdot \int_{\mathbb{R}^{3}} \mathrm{~d} v(\widehat{\zeta g}+\hat{\mathcal{C}}) \mathscr{A}_{\mathfrak{u}}+\int_{\mathbb{R}^{3}} \mathrm{~d} v \frac{1}{2}\left(\left|v_{\mathfrak{u}}\right|^{2}-3\right) \sqrt{\mu}_{\mathfrak{u}} \hat{\mathcal{C}}(k, v) \\
& +\widehat{\zeta \mathfrak{c}}
\end{aligned}
$$

To eliminate the pressure $\hat{P}^{\zeta}$ from (4.33) we apply the Leray projector $\Pi$ defined, in Fourier space, by

We use the short notation

$$
\hat{\Pi}=\mathbf{I}-\frac{k \otimes k}{|k|^{2}} .
$$

$$
N_{\sigma, \beta}(k)=\left\{\begin{array}{l}
\varepsilon[\mathbb{K}+\beta i \mathfrak{u} \cdot k \mathbf{I}] \quad \text { for } \sigma=\mathfrak{v} \text { and } \beta=1 \\
\varepsilon\left[\frac{\kappa}{2}|k|^{2}+\frac{5}{2} i \mathfrak{u} \cdot k\right] \quad \text { for } \sigma=\frac{\kappa}{2} \text { and } \beta=\frac{5}{2}
\end{array}\right.
$$

Thus we get

$$
\hat{\Pi} \hat{b}^{\zeta}=N_{\mathfrak{v}, 1}^{-1} \hat{\Pi} \hat{s} .
$$

Then we multiply the momentum equation by $k$ and divide by $i|k|^{2}$ to obtain

$$
\hat{P}^{\zeta}+\frac{N_{\mathfrak{v}, 1}}{i|k|^{2}} \hat{b}^{\zeta} \cdot k=\frac{k}{i|k|^{2}} \cdot \underline{\hat{s}} .
$$

From the mass equation we have

$$
\hat{b}^{\zeta} \cdot k=-i \hat{s}_{0}-\varepsilon \mathfrak{u} \cdot k \hat{a}^{\zeta}
$$

Hence

$$
\hat{P}+\frac{N_{\mathfrak{v}, 1}}{i|k|^{2}}\left(-i \hat{s}_{0}-\varepsilon \mathfrak{u} \cdot k \hat{a}\right)=\frac{k}{i|k|^{2}} \cdot \underline{\hat{s}}
$$


and reminding that $\hat{a}=\hat{P}-\hat{c}$, we have, for $|\mathfrak{u}|$ sufficiently small,

$$
\hat{P}^{\zeta}=\left(1-\varepsilon \frac{N_{\mathfrak{v}, 1}}{i|k|^{2}} \mathfrak{u} \cdot k\right)^{-1}\left[\frac{N_{\mathfrak{v}, 1}}{i|k|^{2}}\left[i \hat{s}_{0}-\varepsilon \mathfrak{u} \cdot k \hat{c}^{\zeta}\right]+\frac{k}{i|k|^{2}} \cdot \underline{\hat{s}}\right] .
$$

Subtracting the mass equation from the energy equation and using $\hat{a}^{\zeta}=\hat{P}^{\zeta}-\hat{c}^{\zeta}$, the equation for $\hat{c}^{\zeta}$ becomes

$$
\left(N_{\kappa, \frac{5}{2}}\right) \hat{c}^{\zeta}-i \varepsilon \mathfrak{u} \cdot k \hat{P}^{\zeta}=\hat{s}_{4}-\hat{s}_{0}
$$

Replacing the expression of the pressure we obtain

$$
\hat{c}^{\zeta}=(\bar{N})^{-1}\left\{\hat{s}_{4}-\hat{s}_{0}+i \varepsilon \mathfrak{u} \cdot k\left(1-\varepsilon \frac{N_{\mathfrak{v}, 1}}{i|k|^{2}} \mathfrak{u} \cdot k\right)^{-1}\left[\frac{N_{\mathfrak{v}, 1}}{i|k|^{2}} i \hat{s}_{0}+\frac{k}{i|k|^{2}} \cdot \underline{\hat{s}}\right]\right\}
$$

with

$$
\bar{N}=N_{\kappa, \frac{5}{2}}+i \varepsilon^{2}(\mathfrak{u} \cdot k)^{2} \frac{N_{\mathfrak{v}, 1}}{i|k|^{2}}\left(1-\varepsilon \frac{N_{\mathfrak{v}, 1}}{i|k|^{2}} \mathfrak{u} \cdot k\right)^{-1} .
$$

Then $\hat{a}^{\zeta}=\hat{P}^{\zeta}-\hat{c}^{\zeta}$ is obtained by subtracting the expressions of $\hat{P}^{\zeta}$ and $\hat{c}^{\zeta}$ just obtained. Finally, using (4.37) we compute $\left(1-\hat{\Pi} \hat{b}^{\zeta}\right)$.

\section{Estimate of $\left\|\mathbf{P}_{\mathfrak{u}} f\right\|_{3}$}

5.1. Splitting of $\mathbf{P}_{\mathfrak{u}} f$. We define the small $k$ 's cutoff as a smooth function

$$
\mathfrak{j}=\left\{\begin{array}{ll}
1 & \text { for }|k|<1 \\
0 & \text { for }|k|>2
\end{array},\right.
$$

and

$$
\mathfrak{j}^{c}=1-\mathbf{j} .
$$

We will split the source terms $\mathbf{s}=\left(s_{0}, \underline{s}, s_{4}\right)$ into five different contributions $s^{(i)}=\left(s_{0}^{(i)}, \underline{s}^{(i)}, s_{4}^{(i)}\right)$, for $i=1, \ldots, 5$ :

$$
\mathbf{s}=\sum_{i=1}^{5} \mathbf{s}^{(i)}
$$

The source $\mathbf{s}^{(1)}$ corresponds large $k$ 's:

$$
\hat{\mathbf{s}}^{(1)}(k)=\mathbf{j}^{c} \hat{\mathbf{s}} .
$$

Then we split $\hat{\mathcal{C}}(k, v)=\mathcal{F}[f v \cdot \nabla \zeta](k, v)$ as

$$
\hat{\mathcal{C}}=\hat{\mathcal{C}}_{s}+k \cdot \hat{\mathcal{C}}_{r},
$$

with

$$
\hat{\mathcal{C}}_{s}(v)=\hat{\mathcal{C}}(0, v),
$$

and

$$
\hat{\mathcal{C}}_{r}(k, v)=\int_{0}^{1} \mathrm{~d} \lambda \nabla_{k} \hat{\mathcal{C}}(\lambda k, v)
$$

so that

$$
\hat{\mathcal{C}}(k, v)-\hat{\mathcal{C}}(0, v)=\int_{0}^{1} \mathrm{~d} \lambda \frac{d}{d \lambda} \hat{\mathcal{C}}(\lambda k, v)=\int_{0}^{1} \mathrm{~d} \lambda k \cdot \nabla_{k} \hat{\mathcal{C}}(\lambda k, v)=k \cdot \hat{\mathcal{C}}_{r}(k, v) .
$$


We set

$$
\begin{aligned}
& \hat{s}_{0}^{(2)}(k)=\mathfrak{j} \int_{\mathbb{R}^{3}} \mathrm{~d} v \sqrt{\mu}_{\mathfrak{u}} \hat{\mathcal{C}}_{s}(0, v), \\
& \underline{\hat{s}}^{(2)}(k)=\mathfrak{j} \int_{\mathbb{R}^{3}} \mathrm{~d} v v_{\mathfrak{u}} \sqrt{\mu}_{\mathfrak{u}} \hat{\mathcal{C}}_{s}(0, v), \\
& \hat{s}_{4}^{(2)}(k)=\mathfrak{j} \int_{\mathbb{R}^{3}} \mathrm{~d} v \frac{1}{2}\left(\left|v_{\mathfrak{u}}\right|^{2}-3\right) \sqrt{\mu}_{\mathfrak{u}} \hat{\mathcal{C}}_{s}(0, v) . \\
& \hat{s}_{0}^{(3)}(k)=\mathfrak{j}\left[\int_{\mathbb{R}^{3}} \mathrm{~d} v \sqrt{\mu}_{\mathfrak{u}} k \cdot \hat{\mathcal{C}}_{r}(k, v)\right], \\
& \underline{\hat{s}}^{(3)}(k)=\mathfrak{j}\left[-\varepsilon k \otimes k \cdot \int_{\mathbb{R}^{3}} \mathrm{~d} v v\left(\mathbf{I}-\mathbf{P}_{\mathfrak{u}}\right) \hat{f}^{\zeta}(k, v) \mathscr{B}_{\mathfrak{u}}-i \varepsilon k \cdot \int_{\mathbb{R}^{3}} \mathrm{~d} v \hat{\mathcal{C}} \mathscr{B}_{\mathfrak{u}}\right. \\
& \left.+k \cdot \int_{\mathbb{R}^{3}} \mathrm{~d} v v_{\mathfrak{u}} \sqrt{\mu}_{\mathfrak{u}} \hat{\mathcal{C}}_{r}(k, v)\right], \\
& \hat{s}_{4}^{(3)}(k)=\mathfrak{j}\left[-\varepsilon k \otimes k \cdot \int_{\mathbb{R}^{3}} \mathrm{~d} v v\left(\mathbf{I}-\mathbf{P}_{\mathfrak{u}}\right) \hat{f}^{\zeta}(k, v) \mathscr{A}_{\mathfrak{u}}-i \varepsilon k \cdot \int_{\mathbb{R}^{3}} \mathrm{~d} v \hat{\mathcal{C}} \mathscr{A}_{\mathfrak{u}}\right. \\
& \left.+k \cdot \int_{\mathbb{R}^{3}} \mathrm{~d} v \frac{1}{2}\left(\left|v_{\mathfrak{u}}\right|^{2}-3\right) \sqrt{\mu}_{\mathfrak{u}} \hat{\mathcal{C}}_{r}(x, v)\right] . \\
& \hat{s}_{0}^{(4)}(k)=0, \\
& \underline{\hat{s}}^{(4)}(k)=-\mathrm{j} i \varepsilon k \cdot \int_{\mathbb{R}^{3}} \mathrm{~d} v \widehat{\zeta g} \mathscr{B}_{\mathfrak{u}}, \\
& \hat{s}_{4}^{(4)}(k)=-\mathbf{j} \varepsilon i k \cdot \int_{\mathbb{R}^{3}} \mathrm{~d} v \widehat{\zeta g} \mathscr{A}_{\mathfrak{u}} . \\
& \hat{s}_{0}^{(5)}(k)=\mathbf{j} \widehat{\zeta \mathfrak{a}}, \\
& \underline{\hat{s}}^{(5)}(k)=\widehat{j} \widehat{\zeta \mathfrak{b}}, \\
& \hat{s}_{4}^{(5)}(k)=\hat{j} \widehat{\zeta} \mathfrak{c} .
\end{aligned}
$$

For $i=1 \ldots, 5$ we denote by $a^{(i)}, b^{(i)}, c^{(i)}$ the solution to the system (4.32), (4.33), (4.34) with sources $\mathbf{s}^{(i)}$ and by $P^{(i)}=a^{(i)}+c^{(i)}$ the $i$-th contribution to the pressure.

Correspondingly we have the decomposition of $\mathbf{P}_{\mathfrak{u}} f$ into six terms:

$$
\mathbf{P}_{\mathfrak{u}} f=(1-\zeta) \mathbf{P}_{\mathfrak{u}} f+\sum_{i=1}^{5} \mathbf{S}_{i} f
$$

with

$$
\mathbf{S}_{i} f=\sqrt{\mu_{\mathfrak{u}}}\left[a^{(i)}+v_{\mathfrak{u}} \cdot b^{(i)}+\frac{1}{2} c^{(i)}\left(\left|v_{\mathfrak{u}}\right|^{2}-3\right)\right] .
$$

5.2. Estimate of $\mathbf{S}_{1} f$. The components of $\mathbf{S}_{1} \hat{f}$ solve the system

$$
\begin{aligned}
& i k \cdot \hat{b}^{(1)}+i \varepsilon k \cdot \mathfrak{u} \hat{a}^{(1)}=\hat{s}_{0}^{(1)}, \\
& \left.i k \hat{P}^{(1)}+\varepsilon \mathbb{K} b^{\zeta}+i \mathfrak{u} \cdot k\right] \hat{b}^{(1)}=\underline{\hat{s}}^{(1)} \\
& i k \cdot \hat{b}^{(1)}+\varepsilon\left[\kappa|k|^{2}+\frac{3}{2} i k \cdot \mathfrak{u}\right] \hat{c}^{(1)}=\hat{s}_{4}^{(1)},
\end{aligned}
$$


where

$$
\begin{aligned}
\hat{s}_{0}^{(1)} & =\mathfrak{j}^{c} \int_{\mathbb{R}^{3}} \mathrm{~d} v \sqrt{\mu} \hat{\mathcal{C}}(k, v)+\mathfrak{j}^{c} \widehat{\zeta \mathfrak{a}} \\
\underline{\hat{s}}^{(1)} & =\mathfrak{j}^{c}\left[-\varepsilon k \otimes k \cdot \int_{\mathbb{R}^{3}} \mathrm{~d} v v \cdot \mathscr{B}_{\mathfrak{u}}\left(\mathbf{I}-\mathbf{P}_{\mathfrak{u}}\right) \hat{f}^{\zeta}-i \varepsilon k \cdot \int_{\mathbb{R}^{3}} \mathrm{~d} v(\widehat{\zeta g}+\hat{\mathcal{C}}) \mathscr{B}_{\mathfrak{u}}\right. \\
& \left.+\int_{\mathbb{R}^{3}} \mathrm{~d} v v_{\mathfrak{u}} \sqrt{\mu}_{\mathfrak{u}} \hat{\mathcal{C}}(k, v)+\widehat{\zeta \mathfrak{b}}\right] \\
\hat{s}_{4}^{(1)} & =\mathfrak{j}^{c}\left[-\varepsilon k \otimes k \cdot \int_{\mathbb{R}^{3}} \mathrm{~d} v v\left(\mathbf{I}-\mathbf{P}_{\mathfrak{u}}\right) \hat{f}^{\zeta} \mathscr{A}_{\mathfrak{u}}-i \varepsilon k \cdot \int_{\mathbb{R}^{3}} \mathrm{~d} v(\widehat{\zeta g}+\hat{\mathcal{C}}) \mathscr{A}_{\mathfrak{u}}\right. \\
& \left.+\int_{\mathbb{R}^{3}} \mathrm{~d} v \frac{1}{2}\left(\left|v_{\mathfrak{u}}\right|^{2}-3\right) \sqrt{\mu} \hat{\mathcal{C}}(k, v)+\widehat{\zeta \mathfrak{c}}\right] .
\end{aligned}
$$

Lemma 5.1. If $|\mathfrak{u}| \ll 1$, and $g \in L^{2}$, then

$$
\left\|\mathbf{S}_{1} f\right\|_{2} \lesssim \varepsilon^{-1}\left[\left\|\mathbf{P}_{\mathfrak{u}} f\right\|_{6}+\left\|\left(\mathbf{I}-\mathbf{P}_{\mathfrak{u}}\right) f\right\|_{2}\right]+\left\|g \nu^{-\frac{1}{2}}\right\|_{2} .
$$

Proof. We first estimate $\hat{P}^{(1)}$. For this we use the momentum balance in the form (4.19), which for the $\mathbf{S}_{1} \hat{R}$ becomes:

$$
i k \hat{P}^{(1)}+i j^{c} k \cdot \hat{\tau}+i \varepsilon \mathfrak{u} \cdot k \hat{b}^{(1)}=\mathfrak{j}^{c} \widehat{\zeta \mathfrak{b}}+\mathfrak{j}^{c} \int_{\mathbb{R}^{3}} \mathrm{~d} v v_{\mathfrak{u}} \sqrt{\mu}_{\mathfrak{u}} \hat{\mathcal{C}}(k, v) .
$$

We take inner product of this equation with $\frac{k}{i|k|^{2}}$. We obtain

$$
\hat{P}^{(1)}=\mathfrak{j}^{c}\left[-i|k|^{-2} k \cdot \widehat{\zeta \mathfrak{b}}-i|k|^{-2} k \cdot \int_{\mathbb{R}^{3}} \mathrm{~d} v v \hat{\mathcal{C}} \sqrt{\mu}_{\mathfrak{u}}-\varepsilon|k|^{-2} \mathfrak{u} \cdot k \hat{b}^{(1)} \cdot k-|k|^{-2} k \cdot \hat{\tau} \cdot k\right] .
$$

From the definition of $\tau$, (4.5), $\left\|\tau^{(1)}\right\|_{2} \leq\left\|\left(\mathbf{I}-\mathbf{P}_{\mathfrak{u}}\right) f\right\|_{2}$. Moreover from the definition of $\mathcal{C},(4.9)$,

$$
\|\hat{\mathcal{C}}\|_{2}=\|\mathcal{C}\|_{2} \lesssim\left\|\mathbf{P}_{\mathfrak{u}} f\right\|_{L^{2}(\operatorname{supp} \nabla \zeta)}+\left\|\left(\mathbf{I}-\mathbf{P}_{\mathfrak{u}}\right) f\right\|_{L^{2}(\operatorname{supp} \nabla \zeta)} \lesssim\left\|\mathbf{P}_{\mathfrak{u}} f\right\|_{6}+\left\|\left(\mathbf{I}-\mathbf{P}_{\mathfrak{u}}\right) f\right\|_{2} .
$$

Therefore

$$
\left\|\hat{P}^{(1)}\right\|_{2} \lesssim\left\|\mathbf{P}_{\mathfrak{u}} f\right\|_{6}+\left\|\left(\mathbf{I}-\mathbf{P}_{\mathfrak{u}}\right) f\right\|_{2}+\varepsilon|\mathfrak{u}|\left\|\hat{b}^{(1)}\right\|_{2} .
$$

To bound $\hat{b}^{(1)}$, we multiply (4.33) by $\varepsilon^{-1} \mathbb{K}^{1}$ and obtain

$$
\begin{aligned}
\hat{b}^{(1)}=\mathfrak{j}^{c}\left\{\varepsilon ^ { - 1 } \mathbb { K } ^ { - 1 } \left[-i k \hat{P}^{(1)}-i \varepsilon \mathfrak{u} \cdot k \hat{b}^{(1)}-\int_{\mathbb{R}^{3}} \mathrm{~d} v v_{\mathfrak{u}} \sqrt{\mu}_{\mathfrak{u}} \hat{\mathcal{C}}(k, v)\right.\right. \\
\left.\left.\quad+i \varepsilon k \cdot \int_{\mathbb{R}^{3}} \mathrm{~d} v v\left(\mathbf{I}-\mathbf{P}_{\mathfrak{u}}\right) \hat{f} \zeta \mathscr{B}_{\mathfrak{u}}+i \varepsilon k \cdot \int_{\mathbb{R}^{3}} \mathrm{~d} v(\widehat{\zeta g}+\hat{\mathcal{C}}) \mathscr{B}_{\mathfrak{u}}\right]\right\} .
\end{aligned}
$$

Since $|k|>1$, using (4.29) and $|\mathfrak{u}| \ll 1$, we have

$$
\left\|\hat{b}^{(1)}\right\|_{2} \leq \varepsilon^{-1}\left\|\hat{P}^{(1)}\right\|_{2}+\varepsilon^{-1}\left\|\mathbf{P}_{\mathfrak{u}} f\right\|_{6}+\left\|\left(\mathbf{I}-\mathbf{P}_{\mathfrak{u}}\right) f\right\|_{2}+\left\|g \nu^{-\frac{1}{2}}\right\|_{2},
$$

having bounded $\left\|\int \widehat{\zeta g} \mathscr{B}_{\mathfrak{u}}\right\|_{2} \leq\left\|\nu^{-\frac{1}{2}} g\right\|_{2}\left\|\nu^{\frac{1}{2}} \mathscr{B}_{\mathfrak{u}}\right\|_{\infty} \lesssim\left\|\nu^{-\frac{1}{2}} g\right\|_{2}$.

Using (5.2.11) in (5.2.9) and $|\mathfrak{u}| \ll 1$ we have

$$
\left\|\hat{P}^{(1)}\right\|_{2} \lesssim\left\|\mathbf{P}_{\mathfrak{u}} f\right\|_{6}+\left\|\left(\mathbf{I}-\mathbf{P}_{\mathfrak{u}}\right) f\right\|_{2}+\left\|g \nu^{-\frac{1}{2}}\right\|_{2}
$$

Using (5.2.12) in (5.2.11) we obtain

$$
\left\|\hat{b}^{(1)}\right\|_{2} \lesssim \varepsilon^{-1}\left[\left\|\mathbf{P}_{\mathfrak{u}} f\right\|_{6}+\left\|\left(\mathbf{I}-\mathbf{P}_{\mathfrak{u}}\right) f\right\|_{2}\right]+\left\|g \nu^{-\frac{1}{2}}\right\|_{2} .
$$

To estimate $\hat{c}^{(1)}$ we subtract (4.32) from (4.34) and replace $\hat{a}^{(1)}$ with $\hat{P}^{(1)}-\hat{c}^{(1)}$ :

$$
\begin{array}{r}
-i \varepsilon \mathfrak{u} \cdot k\left[\hat{P}^{(1)}-\hat{c}^{(1)}\right]+\int_{\mathbb{R}^{3}} \mathrm{~d} v \sqrt{\mu} \hat{\mathcal{C}}(k, v)+\varepsilon\left[\kappa|k|^{2}+\frac{3}{2} i k \cdot \mathfrak{u}\right] \hat{c}^{(1)}+i \varepsilon k \cdot \int_{\mathbb{R}^{3}} \mathrm{~d} v v\left(\mathbf{I}-\mathbf{P}_{\mathfrak{u}}\right) \hat{f}^{\zeta} \mathscr{A}_{\mathfrak{u}}+ \\
\varepsilon i k \cdot \int_{\mathbb{R}^{3}} \mathrm{~d} v(\widehat{\zeta g}+\hat{\mathcal{C}}) \mathscr{A}_{\mathfrak{u}}=\int_{\mathbb{R}^{3}} \mathrm{~d} v \frac{1}{2}\left(\left|v_{\mathfrak{u}}\right|^{2}-3\right) \sqrt{\mu} \hat{\mathfrak{C}}(k, v) .
\end{array}
$$

Then we proceed as done for $\hat{b}^{(1)}$ and obtain:

$$
\left\|\hat{c}^{(1)}\right\|_{2} \lesssim \varepsilon^{-1}\left[\left\|\mathbf{P}_{\mathfrak{u}} f\right\|_{6}+\left\|\left(\mathbf{I}-\mathbf{P}_{\mathfrak{u}}\right) f\right\|_{2}\right]+\left\|g \nu^{-\frac{1}{2}}\right\|_{2}
$$


From the estimates of $\hat{P}^{(1)}$ and $\hat{c}^{(1)}$ we then obtain also

$$
\left\|\hat{a}^{(1)}\right\|_{2} \lesssim \varepsilon^{-1}\left[\left\|\mathbf{P}_{\mathfrak{u}} f\right\|_{6}+\left\|\left(\mathbf{I}-\mathbf{P}_{\mathfrak{u}}\right) f\right\|_{2}\right]+\left\|g \nu^{-\frac{1}{2}}\right\|_{2} .
$$

Thus, we obtain (5.2.5).

To deal with the system (4.32), (4.33), (4.34) for $|k| \leq 1$ we need several estimates:

Lemma 5.2. Suppose $\mathfrak{u} \neq 0$ and $|k| \leq 1$. Let $N_{\sigma, \beta}(k)=\varepsilon\left[\sigma|k|^{2}+\beta i k \cdot \mathfrak{u}\right]$, for $\sigma>0$ and $\beta>0$. There is $\varrho>0$ such that

(1) For $q \in\left[\frac{3}{2}, 2\right)$

$$
\left\|\mathbf{j} N_{\sigma, \beta}^{-1}\right\|_{q} \lesssim \varepsilon^{-1}|\mathfrak{u}|^{-1+\varrho}
$$

and, for $1<q<\frac{3}{2}$

$$
\left\|\mathbf{j} N_{\sigma, \beta}^{-1}\right\|_{q} \lesssim \varepsilon^{-1}
$$

(2) For $q \in[3,4)$

$$
\left\|\mathrm{j} k N_{\sigma, \beta}^{-1}\right\|_{q} \lesssim \varepsilon^{-1}|\mathfrak{u}|^{-1+\varrho}
$$

and, for $1<q<3$

$$
\left\|\mathrm{j} k N_{\sigma, \beta}^{-1}\right\|_{q} \lesssim \varepsilon^{-1}
$$

$$
\left\|k \otimes k N_{\sigma, \beta}^{-1}\right\|_{\infty} \lesssim \varepsilon^{-1}
$$

Proof. For $\ell \geq 0$ we compute the norm (see 24])

$$
\begin{aligned}
\left\|\varepsilon \mathfrak{j}|k|^{\ell} N_{\sigma, \beta}^{-1}\right\|_{q}^{q} \lesssim & \left.\left.\int_{\mathbb{R}^{3}} \mathrm{~d} k|k|\right|^{q \ell} \mathfrak{j}|\sigma| k\right|^{2}+\left.\beta i k \cdot \mathfrak{u}\right|^{-q} \\
& \leq 2 \pi \sigma^{-q} \int_{0}^{2} \mathrm{~d} r r^{2+q(\ell-2)} \int_{0}^{\pi} \mathrm{d} \theta \sin \theta\left[1+r^{-2} \beta^{2} \sigma^{-2}|\mathfrak{u}|^{2} \cos ^{2} \theta\right]^{-\frac{q}{2}} \\
& =\frac{2 \pi \sigma^{-q}}{\beta \sigma^{-1}|\mathfrak{u}|} \int_{0}^{2} \mathrm{~d} r r^{3+q(\ell-2)} \int_{0}^{r^{-1} \beta|\mathfrak{u}| \sigma^{-1}} \mathrm{~d} z\left[1+z^{2}\right]^{-\frac{q}{2}},
\end{aligned}
$$

with $z=r^{-1} \beta \sigma^{-1}|\mathfrak{u}| \cos \theta$. The integral in $d z$ is finite for $q>1$. The integral in $d r$ is finite for $3+q(\ell-2)>-1$. Hence, for $\ell<2, q<\frac{4}{2-\ell}$. Therefore, if we split the integration on $r$ into $\left\{r \leq|\mathfrak{u}|^{\delta}\right\}$ and $\left\{|\mathfrak{u}|^{\delta}<r \leq 2\right\}$, with $0<\delta<1$ to be chosen, we have the bounds

$$
\begin{gathered}
\int_{0}^{|\mathfrak{u}|^{\delta}} \mathrm{d} r r^{3+q(\ell-2)} \int_{0}^{r^{-1} \beta|\mathfrak{u}| \sigma^{-1}} \mathrm{~d} z\left[1+z^{2}\right]^{-\frac{q}{2}} \lesssim|\mathfrak{u}|^{[4+q(\ell-2)] \delta}, \\
\int_{|\mathfrak{u}|^{\delta}}^{2} \mathrm{~d} r r^{3+q(\ell-2)} \int_{0}^{r^{-1} \beta|\mathfrak{u}| \sigma^{-1}} \mathrm{~d} z\left[1+z^{2}\right]^{-\frac{q}{2}} \leq \int_{|\mathfrak{u}|^{\delta}}^{2} \mathrm{~d} r r^{3+q(\ell-2)} \int_{0}^{|\mathfrak{u}|^{-\delta+1} \beta \sigma^{-1}} \mathrm{~d} z\left[1+z^{2}\right]^{-\frac{q}{2}} \lesssim|\mathfrak{u}|^{1-\delta} .
\end{gathered}
$$

By choosing $\delta=(5+q(2-\ell))^{-1}<1$, we conclude that

$$
\left\|\varepsilon \mathfrak{j}|k|^{\ell} N_{\sigma, \beta}^{-1}\right\|_{q} \lesssim|\mathfrak{u}|^{-\frac{\delta}{q}}=|\mathfrak{u}|^{-1+\varrho}
$$

because $\delta<1$ and $q>1$. Thus, for $\ell=0$ we obtain (5.2.17), for $\ell=1$ we obtain (5.2.19).

If we bound the integrand in $\mathrm{d} \theta$ simply with 1 , as in the Stokes problem, we get instead

$$
\left\|\varepsilon \mathfrak{j}|k|^{\ell} N_{\sigma, \beta}^{-1}\right\|_{q}^{q} \leq 2 \pi^{2} \int_{0}^{2} \mathrm{~d} r r^{2+(\ell-2) q} .
$$

The integral in $\mathrm{d} r$ is finite for $q<\frac{3}{2-\ell}$. For $\ell=0$, the integral is bounded when $q<\frac{3}{2}$, and hence we get (5.2.18); for $\ell=1$, the integral is bounded when $q<3$ and hence we obtain (5.2.20). Clearly $\varepsilon|k|^{2} N_{\sigma, \beta}^{-1} \lesssim 1$ for any $k$, thus we have (5.2.21). 
5.3. Estimate of $\mathbf{S}_{2} f$. The components of $\mathbf{S}_{2} \hat{f}$ solve the system

$$
\begin{aligned}
& i k \cdot \hat{b}^{(2)}+i \varepsilon k \cdot \mathfrak{u} \hat{a}^{(2)}=\hat{s}_{0}^{(2)}, \\
& i k \hat{P}^{(2)}+\varepsilon[\mathbb{K}+i \mathfrak{u} \cdot k] \hat{b}^{(2)}=\underline{\hat{s}}^{(2)} \\
& i k \cdot \hat{b}^{(2)}+\varepsilon\left[\kappa|k|^{2}+\frac{3}{2} i k \cdot \mathfrak{u}\right] \hat{c}^{(2)}=\hat{s}_{4}^{(2)},
\end{aligned}
$$

where

$$
\begin{aligned}
\hat{s}_{0}^{(2)} & =\mathfrak{j} \int_{\mathbb{R}^{3}} \mathrm{~d} v \sqrt{\mu}_{\mathfrak{u}} \hat{\mathcal{C}}(0, v), \\
\underline{\hat{s}}^{(2)} & \left.=\mathfrak{j} \int_{\mathbb{R}^{3}} \mathrm{~d} v v_{\mathfrak{u}} \sqrt{\mu}_{\mathfrak{u}} \hat{\mathcal{C}}(0, v)\right], \\
\hat{s}_{4}^{(2)} & =\mathfrak{j} \int_{\mathbb{R}^{3}} \mathrm{~d} v \frac{1}{2}\left(\left|v_{\mathfrak{u}}\right|^{2}-3\right) \sqrt{\mu}_{\mathfrak{u}} \hat{\mathcal{C}}(0, v),
\end{aligned}
$$

We use the notation $\left.\psi_{0}=\sqrt{\mu}_{\mathfrak{u}}, \psi_{\alpha}=\sqrt{\mu}_{\mathfrak{u}} v_{\mathfrak{u}, \alpha}, \alpha=1, \ldots, 3, \psi_{4}=\frac{1}{\sqrt{6}} \sqrt{\mu}_{\mathfrak{u}}\left(\left|v_{\mathfrak{u}}\right|^{2}-3\right)\right)$, so that $s_{\alpha}^{(2)}=\mathfrak{j}\left(\mathbf{P}_{\mathfrak{u}} \hat{\mathcal{C}}_{s}, \psi_{\alpha}\right)_{L_{v}^{2}}$.

\section{Lemma 5.3.}

$$
\mathbf{P}_{\mathfrak{u}} \hat{\mathcal{C}}_{s}=(2 \pi)^{-\frac{3}{2}} \sum_{\alpha=0}^{4} Q_{\alpha} \psi_{\alpha}
$$

with $\mathbf{Q}=\left(Q_{0}, \ldots Q_{4}\right)$,

$$
Q_{\alpha}=-\int_{\partial \Omega} \mathrm{d} S(x) \int_{\mathbb{R}^{3}} \mathrm{~d} v f v \cdot n(x) \psi_{\alpha}(v)+\int_{\Omega_{1} \backslash \Omega} \mathrm{d} x(1-\zeta) \int_{\mathbb{R}^{3}} \mathrm{~d} v \psi_{\alpha} \mathbf{P}_{\mathfrak{u}} g
$$

Proof. Since $\hat{\mathcal{C}}(0, v)=(2 \pi)^{-\frac{3}{2}} \int \mathrm{d} x f v \cdot \nabla \zeta$, we have

$$
\mathbf{P}_{\mathfrak{u}} \hat{\mathcal{C}}_{s}=(2 \pi)^{-\frac{3}{2}} \sum_{\alpha=0}^{4} \psi_{\alpha} \int_{\Omega^{c}} \mathrm{~d} x \int_{\mathbb{R}^{3}} \mathrm{~d} v f \psi_{\alpha} v \cdot \nabla \zeta .
$$

Since $\nabla \zeta=0$ outside of $\Omega_{1}=\left\{x \in \mathbb{R}^{3} \mid d(x, \Omega)<1\right\}$,

$$
\begin{aligned}
& \int_{\Omega^{c}} \mathrm{~d} x \int_{\mathbb{R}^{3}} \mathrm{~d} v \psi_{\alpha}(v \cdot \nabla \zeta) f=\int_{\Omega_{1} \backslash \Omega} \mathrm{d} x \int_{\mathbb{R}^{3}} \mathrm{~d} v \psi_{\alpha} v \cdot \nabla(\zeta f)-\int_{\Omega_{1} \backslash \Omega} \mathrm{d} x \int_{\mathbb{R}^{3}} \mathrm{~d} v \psi_{\alpha} \zeta v \cdot \nabla f= \\
& \int_{\partial \Omega} \mathrm{d} S(x) \int_{\mathbb{R}^{3}} \mathrm{~d} v v \cdot n(x) \zeta f \psi_{\alpha}(v)+\int_{\partial \Omega_{1}} \mathrm{~d} S(x) \int_{\mathbb{R}^{3}} \mathrm{~d} v v \cdot N(x) \zeta f \psi_{\alpha}(v)-\int_{\Omega_{1} \backslash \Omega} \mathrm{d} x \zeta \int_{\mathbb{R}^{3}} d v \psi_{\alpha} v \cdot \nabla f \\
&=-\int_{\Omega_{1} \backslash \Omega} \mathrm{d} x \zeta \int_{\mathbb{R}^{3}} \mathrm{~d} v \psi_{\alpha} \mathbf{P}_{\mathfrak{u}} g+\int_{\partial \Omega_{1}} \mathrm{~d} S(x) \int_{\mathbb{R}^{3}} \mathrm{~d} v v \cdot N(x) f \psi_{\alpha}(v),
\end{aligned}
$$

where $N(x)$ is the exterior normal to $\partial \Omega_{1}$, because $\zeta=0$ on $\partial \Omega$ and $\zeta=1$ on $\partial \Omega_{1}$ and we have used by (4.1). On the other hand, integrating (4.1) on $\Omega_{1} \backslash \Omega$ we get

$$
\int_{\partial \Omega_{1}} \mathrm{~d} S(x) \int_{\mathbb{R}^{3}} \mathrm{~d} v v \cdot N(x) f \psi_{\alpha}(v)+\int_{\partial \Omega} \mathrm{d} S(x) \int_{\mathbb{R}^{3}} \mathrm{~d} v v \cdot n(x) f \psi_{\alpha}=\int_{\Omega_{1} \backslash \Omega} \mathrm{d} x \int_{\mathbb{R}^{3}} \mathrm{~d} v \psi_{\alpha} \mathbf{P}_{\mathfrak{u}} g,
$$

and hence we obtain

$$
Q_{\alpha}=-\int_{\partial \Omega} \mathrm{d} S(x) \int_{\mathbb{R}^{3}} \mathrm{~d} v f v \cdot n(x) \psi_{\alpha}(v)+\int_{\Omega_{1} \backslash \Omega} \mathrm{d} x(1-\zeta) \int_{\mathbb{R}^{3}} \mathrm{~d} v \psi_{\alpha} \mathbf{P}_{\mathfrak{u}} g
$$

Lemma 5.4 (Estimate of $Q$ 's). If $\left\|\mathbf{P}_{\mathfrak{u}} g\right\|_{L_{l o c}^{6 / 5}}=\left\|\mathbf{P}_{\mathfrak{u}} g\right\|_{L^{6 / 5}\left(\Omega_{1} \backslash \Omega\right)}$ is bounded, then

$$
|\mathbf{Q}| \leq \varepsilon\left(\varepsilon^{-1}\left\|\left(\mathbf{I}-\mathbf{P}_{\mathfrak{u}}\right) f\right\|_{2}+\left\|\mathbf{P}_{\mathfrak{u}} f\right\|_{6}+\left\|\nu^{-\frac{1}{2}} g\right\|_{L^{2}\left(\Omega_{1} \backslash \Omega\right)}\right)+\varepsilon^{\frac{1}{2}}\left\|z_{\gamma}(r)\right\|_{2}+\left\|\mathbf{P}_{\mathfrak{u}} g\right\|_{L_{l o c}^{6 / 5}}
$$


Proof. For any $h$ we have

$$
\int_{\mathbb{R}^{3}} \mathrm{~d} v n \cdot v \sqrt{\mu_{\mathfrak{u}}} h=\int_{\{n \cdot v<0\}} \mathrm{d} v n \cdot v \sqrt{\mu_{\mathfrak{u}}}\left(h-P_{\gamma}^{\mathfrak{u}} h\right) .
$$

Indeed

$$
\begin{aligned}
\int_{\{n \cdot v<0\}} \mathrm{d} v n \cdot v \sqrt{\mu_{\mathfrak{u}}}\left(h-P_{\gamma}^{\mathfrak{u}} h\right)=\int_{\{n \cdot v<0\}} \mathrm{d} v n \cdot v \sqrt{\mu_{\mathfrak{u}}} h \\
\quad-\int_{\{n \cdot v<0\}} \mathrm{d} v n \cdot v \sqrt{2 \pi} \mu(v) \int_{\left\{n \cdot v^{\prime}>0\right\}} \mathrm{d} v^{\prime} v^{\prime} \cdot n \sqrt{\mu_{\mathfrak{u}}} h \\
=\int_{\{n \cdot v<0\}} \mathrm{d} v \cdot v \sqrt{\mu_{\mathfrak{u}}} h+\int_{\left\{n \cdot v^{\prime}>0\right\}} \mathrm{d} v^{\prime} v^{\prime} \cdot n \sqrt{\mu_{\mathfrak{u}}} h=\int_{\mathbb{R}^{3}} \mathrm{~d} v n \cdot v \sqrt{\mu_{\mathfrak{u}}} h,
\end{aligned}
$$

because $\int_{\{n \cdot v<0\}} \mathrm{d} v n \cdot v \sqrt{2 \pi} \mu(v)=-1$.

By (5.3.10) and (1.42),

$$
\begin{aligned}
\int_{\partial \Omega} d S(x) \int_{\mathbb{R}^{3}} d v \sqrt{\mu}_{\mathfrak{u}} f v \cdot n(x)=\int_{\partial \Omega} \mathrm{d} S(x) \int_{\left\{v^{\prime} \cdot n(x)<0\right\}} \mathrm{d} v \sqrt{\mu}_{\mathfrak{u}}\left(f-P_{\gamma}^{\mathfrak{u}} f\right) v \cdot n(x) \\
=\varepsilon^{\frac{1}{2}} \int_{\partial \Omega} \mathrm{d} S(x) \int_{\left\{v^{\prime} \cdot n<0\right\}} \mathrm{d} v \sqrt{\mu}_{\mathfrak{u}} r v^{\prime} \cdot n(x) .
\end{aligned}
$$

Therefore, by (5.3.6),

$$
\left|Q_{0}\right| \leq \varepsilon^{\frac{1}{2}}\left\|z_{\gamma}(r)\right\|_{2}+\left\|\mathbf{P}_{\mathfrak{u}} g\right\|_{L_{\text {loc }}^{6 / 5}}
$$

The other components of $\mathbf{Q}$ are more involved.

Let $\eta(x)=d(x, \partial \Omega)$ be the signed distance of $x \in \mathbb{R}^{3}$ from $\partial \Omega$, positive in $\Omega^{c}$, well defined at least when $|\eta(x)|<\delta$ for some sufficiently small $\delta>0$. Clearly $|\nabla \eta|=1$. We consider the family of smooth closed surfaces $\left\{\mathbb{S}_{\xi}\right\}_{0 \leq \xi<\delta}$, defined as $\mathbb{S}_{\xi}=\left\{x \in \Omega^{c} \mid \eta(x)=\xi\right\}$. We also define, for $x \in \mathbb{S}_{\xi}, n(x)=\nabla \eta(x)$. We have $\mathbb{S}_{0}=\partial \Omega$ and, for any $\xi>0$, the sets $\Omega_{\xi}$ whose boundaries are $\mathbb{S}_{\xi}$ are such that $\Omega_{\xi} \subset \Omega_{\xi^{\prime}}$ if $\xi<\xi^{\prime}$. If we integrate the conservation law on $\Omega_{\xi_{2}} \backslash \Omega_{\xi_{1}}$, since the exterior normal to $\partial \Omega_{\xi_{1}}, n_{1}(x)=-\nabla \eta(x)$, setting

$$
Q_{\xi, \alpha}=-\int_{\mathbb{S}_{\xi}} \mathrm{d} S(x) \int_{\mathbb{R}^{3}} \mathrm{~d} v f \sqrt{\mu_{\mathfrak{u}}} v \cdot n(x) \psi_{\alpha}(v),
$$

by Gauss theorem and (4.1) we obtain

$$
\left|Q_{\xi_{1}, \alpha}-Q_{\xi_{2}, \alpha}\right|=\left|\int_{\Omega_{\xi_{1}} \backslash \Omega_{\xi_{2}}} \mathrm{~d} x \psi_{\alpha} \mathbf{P}_{\mathfrak{u}} g\right| \lesssim\left\|\mathbf{P}_{\mathfrak{u}} g\right\|_{L^{6 / 5}\left(\Omega_{\xi_{1}} \backslash \Omega_{\xi_{2}}\right)}, \quad \alpha=0, \ldots, 4 .
$$

In particular, with

$$
\varpi_{\alpha}=Q_{\alpha}-Q_{0, \alpha}
$$

we have

$$
\left|\varpi_{\alpha}\right| \lesssim\left\|\mathbf{P}_{\mathfrak{u}} g\right\|_{L_{l o c}^{6 / 5}}
$$

and hence, since $|\nabla \eta|=1$, by the coarea formula,

$$
Q_{\alpha}=\varpi_{\alpha}+\delta^{-1} \int_{0}^{\delta} \mathrm{d} \xi Q_{\xi, \alpha}=\varpi_{\alpha}+\delta^{-1} \int_{\Omega_{\delta} \backslash \Omega} \mathrm{d} x \int_{\mathbb{R}^{3}} \mathrm{~d} v f \sqrt{\mu_{\mathfrak{u}}} v \cdot n(x) \psi_{\alpha}(v) .
$$

To estimate $\underline{Q}=\left(Q_{1}, Q_{2}, Q_{3}\right)$, we note that from the decomposition of $f=\sqrt{\mu}_{\mathfrak{u}}\left(a+b \cdot v_{\mathfrak{u}}+\right.$ $\frac{1}{2}\left(\left|v_{\mathfrak{u}}\right|^{2}-3\right)+\left(\overline{\mathbf{I}}-\mathbf{P}_{\mathfrak{u}}\right) f$ and the definitions of $\tau$ and $P$,

$$
\underline{Q}=\underline{\varpi}+\delta^{-1} \int_{\Omega_{\delta} \backslash \Omega} \mathrm{d} x[P n+\tau \cdot n+\varepsilon \mathfrak{u} \cdot n b] .
$$

To get a bound for $P$, let us denote by $\bar{P}$ the average of $P$ on $\Omega_{\delta} \backslash \Omega$ : $\bar{P}=\delta^{-1} \int_{\Omega_{\delta} \backslash \Omega} P d x$. Let $\Phi$ be a vector function such that:

$$
\nabla \cdot \Phi=P-\bar{P} \text { in } \Omega_{\delta} \backslash \Omega, \quad \Phi=0 \text { on } \partial \Omega \cup \partial \Omega_{\delta} .
$$


Such a vector function exists and satisfies the bound (see [19])

$$
\|\Phi\|_{H^{1}\left(\Omega_{\delta} \backslash \Omega\right)} \leq\|P-\bar{P}\|_{L^{2}\left(\Omega_{\delta} \backslash \Omega\right)} .
$$

Taking the inner product of the momentum balance law (4.3)

$$
\nabla(P-\bar{P})+\varepsilon \mathfrak{u} \cdot \nabla b+\nabla \cdot \tau=\mathfrak{b},
$$

by $\Phi$, integrating on $\Omega_{\delta} \backslash \Omega$ and integrating by parts, we obtain

$$
\begin{aligned}
\int_{\Omega_{\delta} \backslash \Omega} \mathfrak{b} \cdot \Phi \mathrm{d} x=-\int_{\Omega_{\delta} \backslash \Omega} \mathrm{d} & {[\nabla \cdot \Phi(P-\bar{P})+\varepsilon \mathfrak{u} \cdot \nabla \Phi \cdot b+\nabla \Phi: \tau] } \\
& +\int_{\partial \Omega \cup \partial \Omega_{\delta}} \mathrm{d} S[\Phi \cdot n(P-\bar{P})+\Phi \otimes n: \tau+\varepsilon \mathfrak{u}(n \cdot \Phi)(b \cdot n)],
\end{aligned}
$$

where $A: B=\sum_{i, j} A_{i, j} B_{i, j}$. The boundary terms vanish because $\Phi=0$ on the boundary. We have

$$
\left|\int_{\Omega_{\delta} \backslash \Omega} \mathfrak{b} \cdot \Phi \mathrm{d} x\right| \leq\|\Phi\|_{6}\|\| \mathfrak{b}\left\|_{L_{l o c}^{6 / 5}} \leq\right\| \mathfrak{b}\left\|_{L_{l o c}^{6 / 5}}\right\| \nabla \Phi \|_{2}
$$

by using Sobolev embedding. Therefore, using $\nabla \cdot \Phi=P-\bar{P}$, we obtain

$$
\begin{aligned}
\|P-\bar{P}\|_{L^{2}\left(\Omega_{\delta} \backslash \Omega\right)}^{2} \leq\|\nabla \Phi\|_{L^{2}\left(\Omega_{\delta} \backslash \Omega\right)}\left(\|\tau\|_{L^{2}\left(\Omega_{\delta} \backslash \Omega\right)}+\varepsilon|\mathfrak{u}|\|b\|_{L^{2}\left(\Omega_{\delta} \backslash \Omega\right)}+\|\mathfrak{b}\|_{L_{l o c}^{6 / 5}}\right) \\
\quad \lesssim\|P-\bar{P}\|_{L^{2}\left(\Omega_{\delta} \backslash \Omega\right)}\left(\left\|\left(\mathbf{I}-\mathbf{P}_{\mathfrak{u}}\right) f\right\|_{L^{2}\left(\Omega_{\delta} \backslash \Omega\right)}+\varepsilon|\mathfrak{u}|\left\|\mathbf{P}_{\mathfrak{u}} f\right\|_{6}+\|\mathfrak{b}\|_{L_{l o c}^{6 / 5}}\right)
\end{aligned}
$$

Hence

$$
\|P-\bar{P}\|_{L^{2}\left(\Omega_{\delta} \backslash \Omega\right)} \lesssim \varepsilon\left(\varepsilon^{-1}\left\|\left(\mathbf{I}-\mathbf{P}_{\mathfrak{u}}\right) f\right\|_{2}+|\mathfrak{u}|\left\|\mathbf{P}_{\mathfrak{u}} f\right\|_{6}\right)+\|\mathfrak{b}\|_{L_{\text {loc }}^{6 / 5}}
$$

Therefore, since $\int_{\Omega_{\delta} \backslash \Omega} \mathrm{d} x \bar{P} n(x)=0$, we obtain

On the other hand,

$$
\left|\int_{\Omega_{\delta} \backslash \Omega} \mathrm{d} x P n\right| \leq \varepsilon\left(\varepsilon^{-1}\left\|\left(\mathbf{I}-\mathbf{P}_{\mathfrak{u}}\right) f\right\|_{2}+|\mathfrak{u}|\left\|\mathbf{P}_{\mathfrak{u}} f\right\|_{6}\right)+\|\mathfrak{b}\|_{L_{l o c}^{\frac{6}{5}}} .
$$

$$
\left|\int_{\Omega_{\delta} \backslash \Omega} \mathrm{d} x \mathfrak{u} \cdot n b\right| \lesssim|\mathfrak{u}| \mid \mathbf{P}_{\mathfrak{u}} f \|_{L^{6}}
$$

and

$$
\left|\int_{\Omega_{\delta} \backslash \Omega} \mathrm{d} x n \cdot \tau\right| \lesssim\left\|\left(\mathbf{I}-\mathbf{P}_{\mathfrak{u}}\right) f\right\|_{L^{2}}
$$

In conclusion

$$
|\underline{Q}| \lesssim \delta \varepsilon\left(\varepsilon^{-1}\left\|\left(\mathbf{I}-\mathbf{P}_{\mathfrak{u}}\right) f\right\|_{2}+|\mathfrak{u}|\left\|\mathbf{P}_{\mathfrak{u}} f\right\|_{6}\right)+\|\mathfrak{b}\|_{L_{l o c}^{6 / 5}}+|\underline{\varpi}| .
$$

For the estimate of $Q_{4}$, we use

$$
\begin{aligned}
Q_{4}=\varpi_{4}+\delta^{-1} \int_{0}^{\delta} \mathrm{d} \xi Q_{\xi, 4}= \\
\varpi_{4}+\delta^{-1} \int_{\Omega_{\delta} \backslash \Omega} \mathrm{d} x \int_{\mathbb{R}^{3}} \mathrm{~d} v \sqrt{\mu_{\mathfrak{u}}} \frac{\left|v_{\mathfrak{u}}\right|^{2}-3}{2} n \cdot v\left\{\left[a+b \cdot v_{\mathfrak{u}}+c \frac{\left|v_{\mathfrak{u}}\right|^{2}-3}{2}\right] \sqrt{\mu_{\mathfrak{u}}}+\left(\mathbf{I}-\mathbf{P}_{\mathfrak{u}}\right) f\right\} \\
\quad=\varpi_{4}+\delta^{-1} \int_{\Omega_{\delta} \backslash \Omega} \mathrm{d} x\left[b \cdot n+\frac{3}{2} \varepsilon \mathfrak{u} \cdot n c+\int_{\mathbb{R}^{3}} \mathrm{~d} v \sqrt{\mu_{\mathfrak{u}}} \frac{\left|v_{\mathfrak{u}}\right|^{2}-3}{2} n \cdot v\left(\mathbf{I}-\mathbf{P}_{\mathfrak{u}}\right) f\right] .
\end{aligned}
$$

To get a bound for $\int_{\Omega_{\delta} \backslash \Omega} \mathrm{d} x b \cdot n$ we note that, from $\left|Q_{0}-Q_{\xi, 0}\right| \leq \varepsilon^{\frac{1}{2}}\left\|z_{\gamma}(r)\right\|_{2}$, integrating on $\xi$ and using again the coarea formula, by (5.3.7)

$$
\left|\delta^{-1} \int_{\Omega_{\delta} \backslash \Omega} \mathrm{d} x[b \cdot n+\varepsilon a \mathfrak{u} \cdot n]-\int_{\partial \Omega} \mathrm{d} S[b \cdot n+\varepsilon \mathfrak{u} \cdot n a]\right| \leq \varepsilon^{\frac{1}{2}}\left\|z_{\gamma}(r)\right\|_{2}+\left\|\mathbf{P}_{\mathfrak{u}} g\right\|_{L_{l o c}^{6 / 5}}
$$

and

$$
=\left|\int_{\partial \Omega} \mathrm{d} S[b \cdot n+\varepsilon \mathfrak{u} \cdot n a]-\varepsilon \int_{\partial \Omega} \mathrm{d} S \mathfrak{u} \cdot n a\right| \leq\left\|z_{\gamma}(r)\right\|_{2},
$$


because $\left|\int_{\partial \Omega} \mathrm{d} S b \cdot n\right|=\left|\int_{\partial \Omega} \int_{\mathbb{R}^{3}} \mathrm{~d} v \sqrt{\mu}_{u} f v \cdot n\right| \leq\left\|z_{\gamma}(r)\right\|_{2}$. Hence $\int_{\partial \Omega} \mathrm{d} S b \cdot n=\varepsilon \int_{\partial \Omega} \mathrm{d} S \mathfrak{u} \cdot n a+$ $O\left(\left\|z_{\gamma}(r)\right\|_{2}\right)$. Now we can replace in (5.3.18) this expression to obtain:

$$
\begin{array}{r}
\left|Q_{4}\right| \leq \delta^{-1}\left|\int_{\Omega_{\delta} \backslash \Omega} \mathrm{d} x\left[\varepsilon \mathfrak{u} \cdot n(c-a)+\int_{\mathbb{R}^{3}} \mathrm{~d} v \sqrt{\mu_{\mathfrak{u}}} \frac{\left|v_{\mathfrak{u}}\right|^{2}-3}{2} n \cdot v\left(\mathbf{I}-\mathbf{P}_{\mathfrak{u}}\right) f\right]\right|+\varepsilon\left|\int_{\partial \Omega} \mathrm{d} S \mathfrak{u} \cdot n a\right| \\
+\varepsilon^{\frac{1}{2}}\left\|z_{\gamma}(r)\right\|_{2}+\left\|\mathbf{P}_{\mathfrak{u}} g\right\|_{L_{l o c}^{6 / 5}}
\end{array}
$$

The first term in the first line is bounded with $\varepsilon\left[|\mathfrak{u}|\|a\|_{6}+|\mathfrak{u}|\|c\|_{6}+\varepsilon^{-1}\left\|\left(\mathbf{I}-\mathbf{P}_{\mathfrak{u}}\right) f\right\|_{2}\right]$. The second is bounded by $\varepsilon|\mathfrak{u}|\left\|\mathbf{P}_{\mathfrak{u}} f\right\|_{L^{2}\left(\partial \Omega \times \mathbb{R}^{3}\right)} \lesssim \varepsilon|\mathfrak{u}|\left(\left\|\mathbf{P}_{\mathfrak{u}} f\right\|_{6}+\varepsilon^{-1}\left\|\left(\mathbf{I}-\mathbf{P}_{\mathfrak{u}}\right) f\right\|_{\nu}+\left\|\nu^{-\frac{1}{2}} g\right\|_{2}\right)$, by using the Ukai trace theorem, Lemma 2.2 .

Lemma 5.5. If $\mathfrak{u} \neq 0$, then there is $\rho>0$ such that, for any $p>2$

$$
\begin{array}{r}
\left\|\mathbf{S}_{2} f\right\|_{p} \lesssim \varepsilon^{-1}|\mathfrak{u}|^{-1+\varrho} \sum_{\alpha=0, \ldots, 4}\left|Q_{\alpha}\right| \lesssim|\mathfrak{u}|^{-1+\varrho}\left(\varepsilon^{-1}\left\|\left(\mathbf{I}-\mathbf{P}_{\mathfrak{u}}\right) f\right\|_{2}+\left\|\mathbf{P}_{\mathfrak{u}} f\right\|_{6}+\left\|g \nu^{-\frac{1}{2}}\right\|_{2}\right) \\
+\varepsilon^{-1}|\mathfrak{u}|^{-1+\varrho}\left[\varepsilon^{\frac{1}{2}}\left\|z_{\gamma}(r)\right\|_{2}+\left\|\mathbf{P}_{\mathfrak{u}} g\right\|_{L_{l o c}^{6 / 5}}\right] .
\end{array}
$$

Proof.

Step 1. Estimate of $\Pi b^{(2)}$ : From (4.36) for the system (4.32), (4.33), (4.34), with $\mathbf{s}=\mathbf{s}^{(2)}$, we have

$$
\hat{\Pi} \hat{b}^{(2)}=\mathfrak{j} N_{\mathfrak{v}, 1}^{-1} \hat{\Pi} \underline{\underline{Q}} .
$$

By (5.2.17), $\mathfrak{j} N_{\mathfrak{v}, 1}^{-1}$ is bounded by $\varepsilon^{-1}|\mathfrak{u}|^{-1+\varrho}$ in $L^{q}\left(\mathbb{R}^{3}\right)$ for $\frac{3}{2} \leq q<2$, and hence

$$
\left\|\hat{\Pi} \hat{b}^{(2)}\right\|_{q} \lesssim \varepsilon^{-1}|\mathfrak{u}|^{-1+\varrho} \underline{Q} .
$$

Step 2. Estimate of $P^{(2)}$ : by using (4.38) for the system (4.32), (4.33), (4.34), we have

$$
\hat{P}^{(2)}=\left(1-\varepsilon \frac{N_{\mathfrak{v}, 1}}{i|k|^{2}} \mathfrak{u} \cdot k\right)^{-1}\left[\frac{N_{\mathfrak{v}, 1}}{i|k|^{2}}\left[i \hat{s}_{0}^{(2)}+\varepsilon \mathfrak{u} \cdot k \hat{c}^{(2)}\right]+\frac{k}{i|k|^{2}} \cdot \underline{\hat{s}}^{(2)}\right] .
$$

Since $\mathfrak{j}|k|^{-q}$ is integrable for any $q<3$, we obtain

$$
\left\|\hat{P}^{(2)}\right\|_{q} \lesssim \varepsilon^{2}\left\|\hat{c}^{(2)}\right\|_{q}+|\underline{Q}|
$$

Step 3. Estimate of $c^{(2)}$ : by using (4.40) for the system (4.32), (4.33), (4.34), we have

$$
\hat{c}^{(2)}=\bar{j}^{-1}\left\{Q_{4}-Q_{0}+i \varepsilon \mathfrak{u} \cdot k\left(1-\varepsilon \frac{N_{\mathfrak{v}, 1}}{i|k|^{2}} \mathfrak{u} \cdot k\right)^{-1} \frac{k}{i|k|^{2}} \cdot \underline{\hat{Q}}\right\},
$$

We remind that from the definition of $\bar{N}$ it follows that $\left|\bar{N}^{-1}\right| \lesssim\left|N_{\kappa, \frac{5}{2}}^{-1}\right|$. Therefore, proceeding as before, we obtain by (5.2.17) for $\frac{3}{2} \leq q<2$,

$$
\left\|\hat{c}^{(2)}\right\|_{q} \lesssim \varepsilon^{-1}|\mathfrak{u}|^{-1+\varrho}\left(\left|Q_{4}\right|+\left|Q_{0}\right|\right)+\varepsilon|\mathfrak{u}| \underline{Q} \mid
$$

and, in consequence,

$$
\left\|\hat{P}^{(2)}\right\|_{q} \lesssim|\mathfrak{u}|^{-1+\varrho}\left(\left|Q_{4}\right|+\left|Q_{0}\right|\right)+|\underline{Q}|
$$

Step 4. Estimate of $\hat{a}^{(2)}$ : Using $\hat{a}^{(2)}=\hat{P}^{(2)}-\hat{c}^{(2)}$, we have

$$
\left\|\hat{a}^{(2)}\right\|_{q} \lesssim \varepsilon^{-1}\left(|\mathfrak{u}|^{-1+\varrho}\left(\left|Q_{4}\right|+\left|Q_{0}\right|\right)+|\underline{Q}|\right) .
$$

Step 5. Estimate of $(1-\hat{\Pi}) \hat{b}^{(2)}$ :

Since $(1-\hat{\Pi}) \hat{b}^{(2)}=k \cdot \hat{b}^{(2)} k|k|^{-2}$, using the mass equation, where $\hat{s}_{0}^{(2)}=\mathrm{j} Q_{0}$, which implies $k \cdot \hat{b}^{(2)}=-\varepsilon k \cdot \mathfrak{u} \hat{a}^{(2)}+i \mathrm{j} Q_{0}$, we have

$$
(1-\hat{\Pi}) \hat{b}^{(2)}=-\varepsilon|k|^{-2} k k \cdot \mathfrak{u} \hat{a}^{(2)}+i|k|^{-2} k \mathrm{j} Q_{0},
$$

and taking the $L^{q}$ norm we have, using Step 4,

$$
\left\|(1-\hat{\Pi}) \hat{b}^{(2)}\right\|_{q} \leq \varepsilon\left\|\hat{a}^{(2)}\right\|_{q}+\left|Q_{0}\right|
$$


Then, together with Step 1 we obtain

$$
\left\|\hat{b}^{(2)}\right\|_{q} \lesssim \varepsilon^{-1}\left(\left|Q_{4}\right|+\left|Q_{0}\right|\right)+|\underline{Q}| .
$$

In conclusion,

$$
\left\|\mathbf{S}_{2} \hat{f}\right\|_{q} \lesssim \varepsilon^{-1}|\mathfrak{u}|^{-1+\varrho} \underline{Q}, \quad \text { for } \frac{3}{2} \leq q<2 .
$$

We remind the Hausdorff-Young inequality: if $1<q \leq 2$ and $\frac{1}{p}+\frac{1}{q}=1$, then

$$
\|f\|_{p} \leq\|\hat{f}\|_{q}
$$

By the Hausdorff-Young inequality then we have (5.3.21) with $p=\frac{q}{q-1}>2$.

5.4. Estimate of $\mathbf{S}_{3} f$. The components of $\mathbf{S}_{3} \hat{R}$ solve the system

$$
\begin{aligned}
& i k \cdot \hat{b}^{(3)}+i \varepsilon k \cdot \mathfrak{u} \hat{a}^{(3)}=\hat{s}_{0}^{(3)}, \\
& i k \hat{P}^{(3)}+\varepsilon[\mathbb{K}+i \mathfrak{u} \cdot k] \hat{b}^{(3)}=\underline{\hat{s}}^{(3)} \\
& i k \cdot \hat{b}^{(3)}+\varepsilon\left[\kappa|k|^{2}+\frac{3}{2} i k \cdot \mathfrak{u}\right] \hat{c}^{(3)}=\hat{s}_{4}^{(3)},
\end{aligned}
$$

where

$$
\begin{aligned}
& \hat{s}_{0}^{(3)}= \mathfrak{j}\left[\int_{\mathbb{R}^{3}} \mathrm{~d} v \sqrt{\mu}_{\mathfrak{u}} k \cdot \hat{\mathcal{C}}_{r}(k, v)\right], \\
& \underline{\hat{s}}^{(3)}(k)= \mathfrak{j}\left[-\varepsilon k \otimes k \cdot \int_{\mathbb{R}^{3}} \mathrm{~d} v v\left(\mathbf{I}-\mathbf{P}_{\mathfrak{u}}\right) \hat{f} \zeta(k, v) \mathscr{B}_{\mathfrak{u}}-i \varepsilon k \cdot \int_{\mathbb{R}^{3}} \mathrm{~d} v \hat{\mathcal{C}} \mathscr{B}_{\mathfrak{u}}\right. \\
&\left.+k \cdot \int_{\mathbb{R}^{3}} \mathrm{~d} v v_{\mathfrak{u}} \sqrt{\mu}_{\mathfrak{u}} \hat{\mathcal{C}}_{r}(k, v)\right], \\
& \hat{s}_{4}^{(3)}(k)= \mathfrak{j}\left[-\varepsilon k \otimes k \cdot \int_{\mathbb{R}^{3}} \mathrm{~d} v v\left(\mathbf{I}-\mathbf{P}_{\mathfrak{u}}\right) \hat{f} \zeta(k, v) \mathscr{A}_{\mathfrak{u}}-i \varepsilon k \cdot \int_{\mathbb{R}^{3}} \mathrm{~d} v \hat{\mathcal{C}} \mathscr{A}_{\mathfrak{u}}\right. \\
&\left.+k \cdot \int_{\mathbb{R}^{3}} \mathrm{~d} v \frac{1}{2}\left(\left|v_{\mathfrak{u}}\right|^{2}-3\right) \sqrt{\mu}_{\mathfrak{u}} \hat{\mathcal{C}}_{r}(x, v)\right] .
\end{aligned}
$$

Lemma 5.6.

$$
\left\|\int_{\mathbb{R}^{3}} \mathrm{~d} v \sqrt{\mu}_{\mathfrak{u}} v_{\mathfrak{u}} \mathfrak{j} k \hat{\mathcal{C}}_{r}\right\|_{\infty} \leq\left\|\mathbf{P}_{\mathfrak{u}} f\right\|_{6}+\left\|\left(\mathbf{I}-\mathbf{P}_{\mathfrak{u}}\right) f\right\|_{\nu}
$$

Proof. Recall from (5.1.7),

$$
\begin{aligned}
\left|\int_{\mathbb{R}^{3}} \mathrm{~d} v \sqrt{\mu}_{\mathfrak{u}} v_{\mathfrak{u}} \mathfrak{j} \cdot \hat{\mathcal{C}}_{r}\right| & =\left|\int_{\mathbb{R}^{3}} \mathrm{~d} v \sqrt{\mu}_{\mathfrak{u}} v_{\mathfrak{u}} \mathfrak{j} \int_{0}^{1} \mathrm{~d} \lambda \frac{d}{d \lambda} \int d x e^{i \lambda k \cdot x} \mathcal{C}(x, v)\right| \\
& =\left|\int_{\mathbb{R}^{3}} \mathrm{~d} v \sqrt{\mu}_{\mathfrak{u}} v_{\mathfrak{u}} \mathfrak{j} \int_{0}^{1} \mathrm{~d} \lambda\{i k \cdot x\} \int \mathrm{d} x e^{i \lambda k \cdot x} \mathcal{C}(x, v)\right| \\
& \leq \int_{\mathbb{R}^{3}} \mathrm{~d} v \sqrt{\mu}_{\mathfrak{u}}\left|v_{\mathfrak{u}}\right|^{2} \int_{\Omega_{1}} \mathrm{~d} x|x| \int_{\mathbb{R}^{3}} \mathrm{~d} v\left(\left|\mathbf{P}_{\mathfrak{u}} f(x, v)\right|+\left|\left(\mathbf{I}-\mathbf{P}_{\mathfrak{u}}\right) f(x, v)\right|\right) \\
& \lesssim\|f\|_{6}+\left\|\left(\mathbf{I}-\mathbf{P}_{\mathfrak{u}}\right) f\right\|_{\nu},
\end{aligned}
$$

because $\operatorname{supp}(\nabla \zeta) \subset \Omega_{1}$.

Lemma 5.7. If $|\mathfrak{u}| \ll 1$ and $\varepsilon \ll 1$,

$$
\left\|\mathbf{S}_{3} f\right\|_{2} \lesssim \varepsilon^{-1}\left\|\mathbf{P}_{\mathfrak{u}} f\right\|_{6}+\varepsilon^{-1}\left\|\left(\mathbf{I}-\mathbf{P}_{\mathfrak{u}}\right) f\right\|_{2} .
$$

Proof. Step 1. Estimate of $\Pi b^{(3)}$ :

From (4.36) for the system (5.4.1), (5.4.2), (5.4.3), with $\mathbf{s}^{(3)}$ given by (5.4.4), we have

$$
\hat{\Pi} \hat{b}^{(3)}=N_{\mathfrak{v}, 1}^{-1} \hat{\Pi}_{\hat{s}^{(3)}},
$$

where

$$
\underline{\hat{s}}^{(3)}=\mathfrak{j}\left[\varepsilon k \otimes k \cdot \int_{\mathbb{R}^{3}} \mathrm{~d} v v\left(\mathbf{I}-\mathbf{P}_{\mathfrak{u}}\right) \hat{f} \zeta \mathscr{B}_{\mathfrak{u}}-i \varepsilon k \cdot \int_{\mathbb{R}^{3}} \mathrm{~d} v \hat{\mathcal{C}} \mathscr{B}_{\mathfrak{u}}+k \cdot \int_{\mathbb{R}^{3}} \mathrm{~d} v v_{\mathfrak{u}} \sqrt{\mu}_{\mathfrak{u}} \hat{\mathcal{C}}_{r}(k, v)\right],
$$


By $(5.2 .20), \mathfrak{j} k N_{\mathfrak{v}, 1}^{-1}$ is bounded by $\varepsilon^{-1}$ in $L^{2}\left(\mathbb{R}^{3}\right)$, and hence

$$
\begin{gathered}
\left\|\mathrm{j} N_{\mathfrak{v}, 1}^{-1} \hat{\Pi} k \cdot \int_{\mathbb{R}^{3}} \mathrm{~d} v v_{\mathfrak{u}} \sqrt{\mu}_{\mathfrak{u}} \hat{\mathcal{C}}_{r}(k, v)\right\|_{2} \lesssim \varepsilon^{-1}\left\|\int_{\mathbb{R}^{3}} \mathrm{~d} v v_{\mathfrak{u}} \sqrt{\mu_{\mathfrak{u}}} k \cdot \hat{\mathcal{C}}_{r}(k, v)\right\|_{\infty} \leq \varepsilon^{-1}\left\|\mathbf{P}_{\mathfrak{u}} f\right\|_{6}+\varepsilon^{-1}\left\|\left(\mathbf{I}-\mathbf{P}_{\mathfrak{u}}\right) f\right\|_{\nu}, \\
\left\|\mathrm{j} \varepsilon N_{\mathfrak{v}, 1}^{-1} \hat{\Pi} k \int_{\mathbb{R}^{3}} \mathrm{~d} v \hat{\mathcal{C}}(k, v) \mathscr{B}_{\mathfrak{u}}\right\|_{2} \lesssim\left\|\int_{\mathbb{R}^{3}} \mathrm{~d} v \hat{\mathcal{C}}(k, v) \mathscr{B}_{\mathfrak{u}}\right\|_{\infty} \lesssim\left\|\mathbf{P}_{\mathfrak{u}} f\right\|_{6}+\left\|\left(\mathbf{I}-\mathbf{P}_{\mathfrak{u}}\right) f\right\|_{\nu},
\end{gathered}
$$

by using (5.4.5).

On the other hand, by Lemma 5.2, $k \otimes k N_{\sigma, \beta}^{-1} \in L^{\infty}$, so

$$
\left\|i \varepsilon \mathfrak{j} N_{\mathfrak{v}, 1}^{-1} \hat{\Pi} k \otimes k \cdot \int_{\mathbb{R}^{3}} \mathrm{~d} v v\left(\mathbf{I}-\mathbf{P}_{\mathfrak{u}}\right) \hat{f}^{\zeta} \mathscr{B}_{\mathfrak{u}}\right\|_{2} \lesssim\left\|\left(\mathbf{I}-\mathbf{P}_{\mathfrak{u}}\right) f\right\|_{\nu}
$$

therefore we have

$$
\left\|\hat{\Pi} \hat{b}^{(3)}\right\|_{2} \lesssim \varepsilon^{-1}\left\|\mathbf{P}_{\mathfrak{u}} f\right\|_{6}+\varepsilon^{-1}\left\|\left(\mathbf{I}-\mathbf{P}_{\mathfrak{u}}\right) f\right\|_{2} .
$$

Step 2. Estimate of $P^{(3)}$ : by using (4.38) for the system (5.4.1), (5.4.2), (5.4.3), we have

$$
\hat{P}^{(3)}=\left(1-\varepsilon \frac{N_{\mathfrak{v}, 1}}{i|k|^{2}} \mathfrak{u} \cdot k\right)^{-1}\left[\frac{N_{\mathfrak{v}, 1}}{i|k|^{2}}\left[i \hat{s}_{0}^{(3)}+\varepsilon \mathfrak{u} \cdot k \hat{c}\right]+\frac{k}{i|k|^{2}} \cdot \underline{\hat{s}}^{(3)}\right] .
$$

Taking the $L^{2}$ norm, for $\varepsilon \ll 1$ we get

$$
\left\|\hat{P}^{(3)}\right\|_{2} \leq \varepsilon^{2}\left\|\hat{c}^{(3)}\right\|_{2}+\left\|\mathbf{P}_{\mathfrak{u}} f\right\|_{6}+\left\|\left(\mathbf{I}-\mathbf{P}_{\mathfrak{u}}\right) f\right\|_{\nu}
$$

Step 3. Estimate of $c^{(3)}$ : by using (4.40) for the system (4.32), (4.33), (4.34), we have

$$
\hat{c}^{(3)}=(\bar{N})^{-1}\left\{s_{4}^{(3)}-s_{0}^{(3)}+i \varepsilon \mathfrak{u} \cdot k\left(1-\varepsilon \frac{N_{\mathfrak{v}, 1}}{i|k|^{2}} \mathfrak{u} \cdot k\right)^{-1}\left[\frac{N_{\mathfrak{v}, 1}}{i|k|^{2}} i \hat{s}_{0}^{(3)}+\frac{k}{i|k|^{2}} \cdot \underline{\hat{s}}^{(3)}\right]\right\},
$$

with

$$
\begin{aligned}
\hat{s}_{4}^{(3)}(k)=\mathfrak{j}\left[\varepsilon k \otimes k \cdot \int_{\mathbb{R}^{3}} \mathrm{~d} v v\left(\mathbf{I}-\mathbf{P}_{\mathfrak{u}}\right) \hat{f}^{\zeta}(k, v) \mathscr{A}_{\mathfrak{u}}-i \varepsilon k \cdot \int_{\mathbb{R}^{3}} \mathrm{~d} v \hat{\mathcal{C}} \mathscr{A}_{\mathfrak{u}}\right. \\
\left.+\int_{\mathbb{R}^{3}} \mathrm{~d} v \frac{1}{2}\left(\left|v_{\mathfrak{u}}\right|^{2}-3\right) \sqrt{\mu}_{\mathfrak{u}} k \cdot \hat{\mathcal{C}}_{r}(k, v)\right] .
\end{aligned}
$$

We remind that from the definition of $\bar{N}$, it follows that $\left|\bar{N}^{-1}\right| \lesssim\left|N_{\kappa, \frac{5}{2}}^{-1}\right|$. Therefore, proceeding as before, we obtain

$$
\left\|\hat{c}^{(3)}\right\|_{2} \lesssim \varepsilon^{-1}\left\|\mathbf{P}_{\mathfrak{u}} f\right\|_{6}+\left\|\left(\mathbf{I}-\mathbf{P}_{\mathfrak{u}}\right) f\right\|_{2} .
$$

and, in consequence,

$$
\left\|\hat{P}^{(3)}\right\|_{2} \leq\left\|\mathbf{P}_{\mathfrak{u}} f\right\|_{6}+\left\|\left(\mathbf{I}-\mathbf{P}_{\mathfrak{u}}\right) f\right\|_{2}
$$

Step 4. Estimate of $\hat{a}^{(3)}$ : Using $\hat{a}^{(3)}=\hat{P}^{(3)}-\hat{c}^{(3)}$, we have

$$
\left\|\hat{a}^{(3)}\right\|_{2} \lesssim \varepsilon^{-1}\left\|\mathbf{P}_{\mathfrak{u}} f\right\|_{6}+\left\|\left(\mathbf{I}-\mathbf{P}_{\mathfrak{u}}\right) f\right\|_{2} .
$$

Step 5. Estimate of $(1-\hat{\Pi}) \hat{b}^{(3)}$ :

Since $(1-\hat{\Pi}) \hat{b}^{(3)}=k \cdot \hat{b}^{(3)} k|k|^{-2}$, using the mass equation which implies $k \cdot \hat{b}^{(3)}=-\varepsilon k \cdot \mathfrak{u} \hat{a}^{(3)}+i \hat{s}_{0}^{(3)}$, we have

$(1-\hat{\Pi}) \hat{b}^{(3)}=-\varepsilon|k|^{-2} k k \cdot \mathfrak{u} \hat{a}^{(3)}+i|k|^{-2} k \hat{s}_{0}^{(3)}-i|k|^{-2} k \cdot\left[\int_{\mathbb{R}^{3}} \mathrm{~d} v v_{\mathfrak{u}} \sqrt{\mu} \hat{\mathcal{C}}_{r}(k, v)+\varepsilon \int_{\mathbb{R}^{3}} \mathrm{~d} v \hat{\mathcal{C}}(k, v) \mathscr{B}_{\mathfrak{u}}\right]$,

and taking the $L^{2}$ norm we have, using Step 4,

$$
\left\|(1-\hat{\Pi}) \hat{b}^{(3)}\right\|_{2} \leq \varepsilon\left\|\hat{a}^{(3)}\right\|_{2}+\left\|\mathbf{P}_{\mathfrak{u}} f\right\|_{6} \lesssim\left\|\mathbf{P}_{\mathfrak{u}} f\right\|_{6}+\left\|\left(\mathbf{I}-\mathbf{P}_{\mathfrak{u}}\right) f\right\|_{\nu} .
$$

Then, together with Step 1 we obtain

$$
\left\|\hat{b}^{(3)}\right\|_{2} \lesssim \varepsilon^{-1}\left\|\mathbf{P}_{\mathfrak{u}} f\right\|_{6}+\left\|\left(\mathbf{I}-\mathbf{P}_{\mathfrak{u}}\right) f\right\|_{\nu} .
$$


5.5. Estimate of $\mathbf{S}_{4} f$. The components of $\mathbf{S}_{4} \hat{f}$ solve the system

$$
\begin{aligned}
& i k \cdot \hat{b}^{(4)}+i \varepsilon k \cdot \mathfrak{u} \hat{a}^{(4)}=\hat{s}_{0}^{(4)}, \\
& i k \hat{P}^{(4)}+\varepsilon[\mathbb{K}+i \mathfrak{u} \cdot k] \hat{b}^{(4)}=\hat{s}^{(4)} \\
& i k \cdot \hat{b}^{(4)}+\varepsilon\left[\kappa|k|^{2}+\frac{3}{2} i k \cdot \mathfrak{u}\right] \hat{c}^{(4)}=\hat{s}_{4}^{(4)},
\end{aligned}
$$

where

$$
\begin{aligned}
& \hat{s}_{0}^{(4)}(k)=0, \\
& \underline{\hat{s}}^{(4)}(k)=-\mathbf{j} i \varepsilon k \cdot \int_{\mathbb{R}^{3}} \mathrm{~d} v \widehat{\zeta g} \mathscr{B}_{\mathfrak{u}}, \\
& \hat{s}_{4}^{(4)}(k)=-\mathbf{j} \varepsilon i k \cdot \int_{\mathbb{R}^{3}} \mathrm{~d} v \widehat{\zeta g} \mathscr{A}_{\mathfrak{u}} .
\end{aligned}
$$

Lemma 5.8. Let $p \geq 2$ and assume $g \in L^{\frac{3 p}{3+p}}$. Then

$$
\left\|\mathbf{S}_{4} f\right\|_{p} \lesssim\left\|\nu^{-\frac{1}{2}} g\right\|_{\frac{3 p}{3+p}}
$$

Proof. We proceed as in the proof of Lemma 5.7

Step 1. Estimate of $\Pi b^{(4)}$ :

From (4.36) for the system (4.32), (4.33), (4.34), with $\mathbf{s}=\mathbf{s}^{(4)}$,

$$
\hat{\Pi} \hat{b}^{(4)}=N_{\mathfrak{v}, 1}^{-1} \hat{\Pi} \underline{\hat{s}}^{(4)} .
$$

Since for the multipliers $k N_{b, 1}^{-1} \hat{\Pi} k$ direct computations yields

$$
\partial_{k}^{l}\left\{\varepsilon k N_{b, 1}^{-1} \hat{\Pi} k\right\} \lesssim_{l}|k|^{-l},
$$

with constants independent of $\varepsilon$, by Mihlin-Hormander's [26, 18, multiplier theorem, we deduce

$$
\left\|\nabla \Pi b^{(4)}\right\|_{\frac{3 p}{3+p}}=\left\|\nabla \mathcal{F}^{-1}\left[N_{\mathfrak{v}, 1}^{-1} \hat{\Pi}\left(\varepsilon \dot{j} i k \cdot \int_{\mathbb{R}^{3}} \mathrm{~d} v \widehat{\zeta g} \mathscr{B}_{\mathfrak{u}}\right)\right]\right\|_{\frac{3 p}{3+p}} \lesssim\left\|\nu^{-\frac{1}{2}} g\right\|_{\frac{3 p}{3+p}},
$$

by the Sobolev estimate

$$
\left\|\Pi b^{(4)}\right\|_{p} \lesssim\left\|\nabla \Pi b^{(4)}\right\|_{\frac{3 p}{3+p}} \lesssim\left\|\nu^{-\frac{1}{2}} g\right\|_{\frac{3 p}{3+p}} .
$$

Step 2. Estimate of $P^{(4)}$ : by using (4.38) for the system (4.32), (4.33), (4.34), we have

$$
\hat{P}^{(4)}=\left(1+\varepsilon \frac{N_{\mathfrak{v}, 1}}{i|k|^{2}} \mathfrak{u} \cdot k\right)^{-1}\left[\frac{N_{\mathfrak{v}, 1}}{i|k|^{2}}\left[\varepsilon \mathfrak{u} \cdot k \hat{c}^{(4)}\right]+\frac{k}{i|k|^{2}} \cdot \underline{\hat{s}}^{(5)}\right],
$$

from which we get

$$
\left\|P^{(4)}\right\|_{p} \lesssim \varepsilon^{2}\left\|c^{(4)}\right\|_{p}+\varepsilon\left\|\nu^{-\frac{1}{2}} g\right\|_{\frac{3 p}{3+p}} .
$$

Step 3. Estimate of $c^{(4)}$ : by using (4.40) for the system (4.32), (4.33), (4.34), we have

$$
\hat{c}^{(4)}=(\bar{N})^{-1}\left\{s_{4}^{(4)}+i \varepsilon \mathfrak{u} \cdot k\left(1+\varepsilon \frac{N_{\mathfrak{v}, 1}}{i|k|^{2}} \mathfrak{u} \cdot k\right)^{-1} \frac{k}{i|k|^{2}} \cdot \underline{\hat{s}}^{(4)}\right\},
$$

with

This implies

$$
s_{4}^{(4)}=\varepsilon i k \cdot \int_{\mathbb{R}^{3}} \mathrm{~d} v \widehat{\zeta g} \mathscr{A}_{\mathfrak{u}}
$$

In consequence

$$
\left\|c^{(4)}\right\|_{p} \leq\left\|\nu^{-\frac{1}{2}} g\right\|_{\frac{3 p}{3+p}} .
$$

$$
\left\|\hat{P}^{(4)}\right\|_{p} \leq \varepsilon\left\|\nu^{-\frac{1}{2}} g\right\|_{\frac{3 p}{3+p}} .
$$

Step 4. Estimate of $\hat{a}^{(4)}$ : Using $\hat{a}^{(4)}=\hat{P}^{(4)}-\hat{c}^{(4)}$, we have

$$
\left\|a^{(4)}\right\|_{p} \lesssim\left\|\nu^{-\frac{1}{2}} g\right\|_{\frac{3 p}{3+p}} .
$$


Step 5. Estimate of $(1-\hat{\Pi}) \hat{b}^{(4)}$ : Since $(1-\hat{\Pi}) \hat{b}^{(4)}=k \cdot b_{l}^{(4)} k|k|^{-2}$, using the equation for the mass we have $(1-\hat{\Pi}) \hat{b}^{(4)}=-\varepsilon|k|^{-2} k k \cdot \mathfrak{u} \hat{a}^{(4)}$, and hence, by Step 4

$$
\left\|(1-\Pi) b^{(4)}\right\|_{p} \leq \varepsilon\left\|a^{(4)}\right\|_{p} \lesssim \varepsilon\left\|\nu^{-\frac{1}{2}} g\right\|_{\frac{3 p}{3+p}} .
$$

Then, together with Step 1 we obtain

$$
\left\|b^{(4)}\right\|_{p} \lesssim\left\|\nu^{-\frac{1}{2}} g\right\|_{\frac{3 p}{3+p}} .
$$

5.6. Estimate of $\mathbf{S}_{5} f$. The components of $\mathbf{S}_{5} \hat{f}$ solve the system

$$
\begin{aligned}
& i k \cdot \hat{b}^{(5)}+i \varepsilon k \cdot \mathfrak{u} \hat{a}^{(5)}=\hat{s}_{0}^{(5)}, \\
& i k \hat{P}^{(5)}+\varepsilon[\mathbb{K}+i \mathfrak{u} \cdot k] \hat{b}^{(5)}=\underline{\hat{s}}^{(5)} \\
& i k \cdot \hat{b}^{(5)}+\varepsilon\left[\kappa|k|^{2}+\frac{3}{2} i k \cdot \mathfrak{u}\right] \hat{c}^{(5)}=\hat{s}_{4}^{(5)},
\end{aligned}
$$

where

$$
\begin{aligned}
\hat{s}_{0}^{(5)}(k) & =\mathfrak{j} \int_{\mathbb{R}^{3}} \mathrm{~d} v \sqrt{\mu_{\mathfrak{u}}} \widehat{\zeta \mathbf{P}_{\mathfrak{u}} g}, \\
\hat{\underline{s}}^{(5)}(k) & =\mathfrak{j} \int_{\mathbb{R}^{3}} \mathrm{~d} v \sqrt{\mu_{\mathfrak{u}}} v \widehat{\zeta \mathbf{P}_{\mathfrak{u}} g}, \\
\hat{s}_{4}^{(5)}(k) & =\mathfrak{j} \int_{\mathbb{R}^{3}} \mathrm{~d} v \sqrt{\mu}_{\mathfrak{u}} \frac{1}{2}\left(|v|^{2}-3\right) \widehat{\zeta \mathbf{P}_{\mathfrak{u}} g} .
\end{aligned}
$$

Lemma 5.9. Let $p>2$. Suppose that $\zeta \mathbf{P}_{\mathfrak{u}} g \in L^{q}, 1<q<\frac{2 p}{p+2}$. Then there is $\rho>0$ such that

$$
\left\|\mathbf{S}_{5} f\right\|_{p} \lesssim \varepsilon^{-1}|\mathfrak{u}|^{-1+\varrho}\left\|\zeta \mathbf{P}_{\mathfrak{u}} g\right\|_{q} .
$$

Proof. By Hausdorff-Young inequality (5.3.31),

$$
\left\|\mathbf{S}_{5} f\right\|_{p} \lesssim\left\|\widehat{\mathbf{S}_{5} f}\right\|_{\frac{p}{p-1}}
$$

We have

$$
\hat{\Pi} \hat{b}^{(5)}=N_{\mathfrak{v}, 1}^{-1} \hat{\Pi} \mathfrak{j} \int_{\mathbb{R}^{3}} \mathrm{~d} v \sqrt{\mu}_{\mathfrak{u}} v \widehat{\zeta \mathbf{P}_{\mathfrak{u}} g} .
$$

Therefore, if $1<r$ and $r \frac{p}{p-1}<2$, so that we can use with (5.2.17), then, with $\frac{1}{q}+\frac{1}{q^{\prime}}=1$, we have

$$
\left\|\hat{\Pi} \hat{b}^{(5)}\right\|_{\frac{p}{p-1}} \leq\left\|N_{\mathfrak{v}, 1}^{-1}\right\|_{r \frac{p}{p-1}}\left\|\widehat{\zeta \mathbf{P}_{\mathfrak{u}} g}\right\|_{\frac{p}{p-1} \frac{r}{r-1}} \lesssim \varepsilon^{-1}|\mathfrak{u}|^{-1+\varrho}\left\|\widehat{\zeta \mathbf{P}_{\mathfrak{u}} g}\right\|_{q^{\prime}} \lesssim \varepsilon^{-1}|\mathfrak{u}|^{-1+\varrho}\left\|\zeta \mathbf{P}_{\mathfrak{u}} g\right\|_{q},
$$

where $q^{\prime}=\frac{p}{p-1} \frac{r}{r-1}$, having used (5.2.17) in the second inequality and again the Hausdorf-Young inequality in the last step. Since $\frac{p-1}{r}>\frac{p}{2}$, then $q=\frac{p r}{p+r-1}=\frac{p}{\frac{p-1}{r}+1}<\frac{2 p}{p+2}$. Since $r>1$, then $q>1$.

\subsection{Proof of Theorem 1.5.}

Proposition 5.10. If $\mathfrak{u} \neq 0$ and $\varepsilon \ll 1$, then there is $\rho>0$ such that,

$$
\begin{array}{r}
\varepsilon^{\frac{1}{2}}\left\|\mathbf{P}_{\mathfrak{u}} f\right\|_{3} \lesssim\left\|\mathbf{P}_{\mathfrak{u}} f\right\|_{6}+|\mathfrak{u}|^{-1+\varrho} \varepsilon^{-1}\left\|\left(\mathbf{I}-\mathbf{P}_{\mathfrak{u}}\right) f\right\|_{2}+o(1)\left\|\nu^{-\frac{1}{2}} g\right\|_{L^{2}\left(\Omega_{1} \backslash \Omega\right)}+\varepsilon^{\frac{1}{2}}\left\|\nu^{-\frac{1}{2}} g\right\|_{\frac{3}{2}} \\
+|\mathfrak{u}|^{-1+\varrho}\left[\varepsilon^{-\frac{1}{2}}\left\|z_{\gamma}(r)\right\|_{2}+\varepsilon^{-1}\left\|\mathbf{P}_{\mathfrak{u}} g\right\|_{L^{\frac{6}{5}}}\right] .
\end{array}
$$

Proof. To get the $L^{3}$ bound of $\mathbf{P}_{\mathfrak{u}} f$ we proceed as follows: we look at the problem in $\mathbb{R}^{3}$ by passing to the cut-offed problem. Thus we obtain $\mathbf{P}_{\mathfrak{u}} f=(1-\zeta) \mathbf{P}_{\mathfrak{u}} f+\sum_{i=1}^{5} \mathbf{S}_{i} f$. Since $1-\zeta(x)=0$ if $x \notin \Omega_{1}=\{x \mid d(x, \Omega) \leq 1\},\left\|(1-\zeta) \mathbf{P}_{\mathfrak{u}} f\right\|_{3} \lesssim\left\|\mathbf{P}_{\mathfrak{u}} f\right\|_{6}$. For the other terms we use the previous lemmas. 
The bounds in previous subsettions are too singular in $\varepsilon$ for our purposes. Therefore, we take advantage of the uniform-in- $\varepsilon$ estimate of $\left\|\zeta \mathbf{P}_{\mathfrak{u}} f\right\|_{6}$ to improve the estimate of $\left\|\zeta \mathbf{P}_{\mathfrak{u}} f\right\|_{3}$ by means of interpolation between the $L^{6}$ norm and some lower norm. Since

$$
\left\|\sum_{i=1}^{5} \mathbf{S}_{i} f\right\|_{6} \leq\left\|\mathbf{P}_{\mathfrak{u}} f\right\|_{6}
$$

we have

$$
\left\|\mathbf{S}_{1} f+\mathbf{S}_{3} f\right\|_{6} \leq\left\|\mathbf{P}_{\mathfrak{u}} f\right\|_{6}+\left\|\mathbf{S}_{2} f\right\|_{6}+\left\|\mathbf{S}_{4} f\right\|_{6}+\left\|\mathbf{S}_{5} f\right\|_{6} .
$$

Therefore, using (5.3.21), (5.5.7) and (5.6.5) with $p=6$, we obtain:

$$
\begin{aligned}
\left\|\mathbf{S}_{1} f+\mathbf{S}_{3} f\right\|_{6} & \lesssim\left\|\mathbf{P}_{\mathfrak{u}} f\right\|_{6}+|\mathfrak{u}|^{-1+\varrho}\left(\varepsilon^{-1}\left\|\left(\mathbf{I}-\mathbf{P}_{\mathfrak{u}}\right) f\right\|_{2}+\left\|\mathbf{P}_{\mathfrak{u}} f\right\|_{6}+\left\|g \nu^{-\frac{1}{2}}\right\|_{2}\right) \\
& +\varepsilon^{-1}|\mathfrak{u}|^{-1+\varrho}\left[\varepsilon^{\frac{1}{2}}\left\|z_{\gamma}(r)\right\|_{2}+\left\|\mathbf{P}_{\mathfrak{u}} g\right\|_{L_{\text {loc }}^{6 / 5}}+\left\|\nu^{-\frac{1}{2}} g\right\|_{2}+\varepsilon^{-1}|\mathfrak{u}|^{-1+\varrho}\left\|\zeta \mathbf{P}_{\mathfrak{u}} g\right\|_{\frac{6}{5}}\right. \\
& \lesssim\left\|\mathbf{P}_{\mathfrak{u}} f\right\|_{6}+|\mathfrak{u}|^{-1+\varrho} \varepsilon^{-1} \|\left(\mathbf{I}-\mathbf{P}_{\mathfrak{u}} f\left\|_{2}+|\mathfrak{u}|^{-1+\varrho}\right\| g \nu^{-\frac{1}{2}} \|_{2}\right. \\
& +|\mathfrak{u}|^{-1+\varrho}\left[\varepsilon^{-\frac{1}{2}}\left\|z_{\gamma}(r)\right\|_{2}+\varepsilon^{-1}\left\|\mathbf{P}_{\mathfrak{u}} g\right\|_{L_{l o c}^{6 / 5}}\right]
\end{aligned}
$$

Note that only the last line is singular in $\varepsilon$, but we will apply the inequality in a situation where $z_{\gamma}(r)$ and $\mathbf{P}_{\mathfrak{u}} g$ are small in $\varepsilon$.

For $\mathbf{S}_{1} f+\mathbf{S}_{3} f$, by (5.2.5) and (5.4.6) (by interpolation $\left(\|f\|_{r} \leq\|f\|_{p}^{\theta}\|f\|_{q}^{1-\theta}\right.$ with $r^{-1}=$ $\left.\left.\theta p^{-1}+(1-\theta) q^{-1}\right)\right)$ we obtain, with $r=3, p=2, q=6$ and $\theta=\frac{1}{2}$,

$$
\begin{aligned}
& \quad \varepsilon^{\frac{1}{2}}\left\|\mathbf{S}_{1} f+\mathbf{S}_{3} f\right\|_{3} \leq\left(\varepsilon\left\|\mathbf{S}_{1} f+\mathbf{S}_{3} f\right\|_{2}\right)^{\frac{1}{2}}\left\|\mathbf{S}_{1} f+\mathbf{S}_{3} f\right\|_{6}^{\frac{1}{2}} \lesssim\left[\left\|\mathbf{P}_{\mathfrak{u}} f\right\|_{6}^{\frac{1}{2}}+\left\|\left(\mathbf{I}-\mathbf{P}_{\mathfrak{u}}\right) f\right\|_{2}^{\frac{1}{2}}+\varepsilon^{\frac{1}{2}}\left\|\nu^{-\frac{1}{2}} g\right\|_{2}^{\frac{1}{2}}\right] \times \\
& {\left[\left\|\mathbf{P}_{\mathfrak{u}} f\right\|_{6}+|\mathfrak{u}|^{-1+\varrho} \varepsilon^{-1}\left\|\left(\mathbf{I}-\mathbf{P}_{\mathfrak{u}}\right) f\right\|_{2}+|\mathfrak{u}|^{-1+\varrho}\left\|g \nu^{-\frac{1}{2}}\right\|_{2}+|\mathfrak{u}|^{-1+\varrho}\left[\varepsilon^{-\frac{1}{2}}\left\|z_{\gamma}(r)\right\|_{2}+\varepsilon^{-1}\left\|\mathbf{P}_{\mathfrak{u}} g\right\|_{L_{l o c}^{6 / 5}}\right]\right]^{\frac{1}{2}}} \\
& \lesssim\left\|\mathbf{P}_{\mathfrak{u}} f\right\|_{6}+|\mathfrak{u}|^{-1+\varrho} \varepsilon^{-1}\left\|\left(\mathbf{I}-\mathbf{P}_{\mathfrak{u}}\right) f\right\|_{2}+\left(\varepsilon+|\mathfrak{u}|^{-1+\varrho}\right)\left\|\nu^{-\frac{1}{2}} g\right\|_{2}+|\mathfrak{u}|^{-1+\varrho}\left[\varepsilon^{-\frac{1}{2}}\left\|z_{\gamma}(r)\right\|_{2}+\varepsilon^{-1}\left\|\mathbf{P}_{\mathfrak{u}} g\right\|_{L_{\text {loc }}^{6 / 5}}\right] .
\end{aligned}
$$

As for $\mathbf{S}_{4} f$, we have from Lemma 5.8 with $p=3$,

$$
\varepsilon^{\frac{1}{2}}\left\|\mathbf{S}_{4} f\right\|_{3} \lesssim \varepsilon^{\frac{1}{2}}\left\|\nu^{-\frac{1}{2}} g\right\|_{\frac{3}{2}} .
$$

For $\mathbf{S}_{5} f$ we use Lemma 5.9 with $p=3$ and hence $1<q<\frac{6}{5}$.

By (5.3.21), by interpolation we obtain,

$$
\begin{aligned}
\varepsilon^{\frac{1}{2}}\left\|\mathbf{S}_{2} f\right\|_{3} \lesssim \varepsilon^{\frac{1}{2}}\left[|\mathfrak{u}|^{-1+\varrho}\left(\varepsilon^{-1}\left\|\left(\mathbf{I}-\mathbf{P}_{\mathfrak{u}}\right) f\right\|_{2}+\left\|\mathbf{P}_{\mathfrak{u}} f\right\|_{6}+\left\|\nu^{-\frac{1}{2}} g\right\|_{2}\right)\right. \\
\left.\quad+\varepsilon^{-1}|\mathfrak{u}|^{-1+\varrho}\left[\varepsilon^{\frac{1}{2}}\left\|z_{\gamma}(r)\right\|_{2}+\left\|\mathbf{P}_{\mathfrak{u}} g\right\|_{L_{\text {loc }}^{6 / 5}}\right]\right]^{\theta}\left\|\mathbf{S}_{2} f\right\|_{6}^{(1-\theta)},
\end{aligned}
$$

with $\theta$ such that $\frac{1}{3}=\theta p^{-1}+\frac{1}{6}(1-\theta)$, and hence $\theta=\frac{1}{2}^{+}$when $p=2^{+}$. Therefore, by Young inequality,

$$
\begin{aligned}
\varepsilon^{\frac{1}{2}}\left\|\mathbf{S}_{2} f\right\|_{3} \lesssim \varepsilon^{\frac{1}{2}}|\mathfrak{u}|^{-1+\varrho}\left(\varepsilon^{-1}\left\|\left(\mathbf{I}-\mathbf{P}_{\mathfrak{u}}\right) f\right\|_{2}+\left\|\mathbf{P}_{\mathfrak{u}} f\right\|_{6}\right. & \left.+\left\|\nu^{-\frac{1}{2}} g\right\|_{2}\right) \\
& +|\mathfrak{u}|^{-1+\rho}\left[\left\|z_{\gamma}(r)\right\|_{2}+\varepsilon^{-\frac{1}{2}}\left\|\mathbf{P}_{\mathfrak{u}} g\right\|_{L_{\text {loc }}^{6 / 5}}\right] .
\end{aligned}
$$

Combining the estimates we obtain (5.7.1).

Now we have all the information needed to prove Theorem 1.5

Proof of Theorem 1.5. To bound the first two terms of $[[f]]_{\beta, \beta^{\prime}}$, we use Proposition 2.6 . Then we use Proposition 2.7 in 3.4 .

$$
\begin{array}{r}
(1-o(1))\left\|\mathbf{P}_{\mathfrak{u}} f\right\|_{6} \lesssim\left(1+o(1)+|\mathfrak{u}|^{-2+2 \rho}\right)\left[\varepsilon^{-1}\left\|\left(\mathbf{I}-\mathbf{P}_{\mathfrak{u}}\right) f\right\|_{\nu}+\varepsilon^{-\frac{1}{2}}\left|\left(1-P_{\gamma}^{\mathfrak{u}}\right) f\right|_{2,+}\right]+\left\|\nu^{-\frac{1}{2}} g\right\|_{2}+\varepsilon^{\frac{1}{2}}|r|_{\infty} \\
+o(1)\left[\varepsilon^{\frac{1}{2}}|w r|_{\infty}+\varepsilon^{\frac{3}{2}}\left\|\langle v\rangle^{-1} w g\right\|_{\infty}\right] .
\end{array}
$$


Using this in (2.8), if $|\mathfrak{u}|$ is so small that $|\mathfrak{u}|\left(1+o(1)+|\mathfrak{u}|^{-1+\rho}\right)(1-o(1))^{-1}<\frac{1}{2}$, we obtain

$$
\begin{aligned}
& \varepsilon^{-2}\left\|\left(\mathbf{I}-\mathbf{P}_{\mathfrak{u}}\right) f\right\|_{\nu}^{2}+\varepsilon^{-1}\left|\left(1-P_{\gamma}^{\mathfrak{u}}\right) f\right|_{2,+}^{2} \lesssim\left\|\nu^{-\frac{1}{2}}\left(\mathbf{I}-\mathbf{P}_{\mathfrak{u}}\right) g\right\|_{2}^{2}+|\mathfrak{u}|\left[\left\|\nu^{-\frac{1}{2}} g\right\|_{2}+\varepsilon^{\frac{1}{2}}|r|_{\infty} \quad\right. \text { (5.7.10) } \\
& \left.+o(1)\left[\varepsilon^{\frac{1}{2}}|w r|_{\infty}+\varepsilon^{\frac{3}{2}}\left\|\langle v\rangle^{-1} w g\right\|_{\infty}\right]\right]+|r|_{2,-}^{2}+(\varepsilon|\mathfrak{u}|)^{-1}\left\|z_{\gamma}(r)\right\|_{2}^{2}+\varepsilon^{-2}|\mathfrak{u}|^{-2}\left\|\mathbf{P}_{\mathfrak{u}} g\right\|_{\frac{6}{5}}^{2}+\left\|\mathbf{P}_{\mathfrak{u}} g\right\|_{2}^{2} .
\end{aligned}
$$

Using this in (3.4) we obtain a similar bound for $\left\|\mathbf{P}_{\mathfrak{u}} f\right\|_{6}$ :

$$
\begin{aligned}
\left\|\mathbf{P}_{\mathfrak{u}} f\right\|_{6} \lesssim\left\|\nu^{-\frac{1}{2}}\left(\mathbf{I}-\mathbf{P}_{\mathfrak{u}}\right) g\right\|_{2}^{2} & +|\mathfrak{u}|\left[\left\|\nu^{-\frac{1}{2}} g\right\|_{2}+\varepsilon^{\frac{1}{2}}|r|_{\infty}+o(1)\left[\varepsilon^{\frac{1}{2}}|w r|_{\infty}+\varepsilon^{\frac{3}{2}}\left\|\langle v\rangle^{-1} w g\right\|_{\infty}\right]\right] \\
& +|r|_{2,-}^{2}+(\varepsilon|\mathfrak{u}|)^{-1}\left\|z_{\gamma}(r)\right\|_{2}^{2}+\varepsilon^{-2}|\mathfrak{u}|^{-2}\left\|\mathbf{P}_{\mathfrak{u}} g\right\|_{\frac{6}{5}}^{2}+\left\|\mathbf{P}_{\mathfrak{u}} g\right\|_{2}^{2} .
\end{aligned}
$$

Using (5.7.10) and (5.7.11) in (2.11) we get a similar bound for $\varepsilon^{\frac{1}{2}}\|w f\|_{\infty}$. Finally, using (5.7.10) and (5.7.11) in (5.7.1) we obtain the bound on $\varepsilon^{\frac{1}{2}}\left\|\mathbf{P}_{\mathfrak{u}} f\right\|_{3}$. Rearranging the terms we obtain (1.43).

\section{Construction of the positive solution to the non Linear Problem}

6.1. Positivity scheme. In order to construct a non negative solution to the problem (1.1) we use a modification of the argument introduced in [1].

We define $F^{+}=\max \{F, 0\}$ and $F^{-}=\max \{-F, 0\}$, so that $F=F^{+}-F^{-}$. Consider the system

$$
\begin{aligned}
& v \cdot \nabla F=\varepsilon^{-1}\left[Q\left(F^{+}, F^{+}\right)-2 Q\left(\mu_{\mathfrak{u}}, F^{-}\right)\right] \text {in } \Omega^{c}, \\
& \left.F\right|_{-}=\mathcal{P}_{\gamma}^{w}\left(F^{+}\right) \text {on } \partial \Omega, \quad \lim _{|x| \rightarrow \infty} F=\mu_{\mathfrak{u}} .
\end{aligned}
$$

Proposition 6.1. Let $F \in L^{\infty}$ solve problem (6.1.1), (6.1.2). Then $F^{-}=0$ and $F^{+}$solves the Boltzmann equation.

Remark 6.2. Since $F^{-}=0, F$ is non negative.

Proof. In fact, the equation for $F^{-}$is

$$
-v \cdot \nabla F^{-}=\varepsilon^{-1} \mathbf{1}_{F^{-} \neq 0}\left[Q^{+}\left(F^{+}, F^{+}\right)-Q\left(\mu_{\mathfrak{u}}, F^{-}\right)-Q\left(F^{-}, \mu_{\mathfrak{u}}\right)\right],\left.\quad F^{-}\right|_{-}=0 .
$$

because $F^{-} \neq 0$ implies $F^{+}=0$, and hence the term $\mathbf{1}_{F^{-} \neq 0} Q^{-}\left(F^{+}, F^{+}\right)=\mathbf{1}_{F^{-} \neq 0} F^{+} \nu\left(F^{+}\right)=0$. Moreover, since $F>0$ on $\gamma_{-}$, if follows that $F^{-}=0$ on $\gamma_{-}$. Since $F \rightarrow \mu_{\mathfrak{u}}>0$ as $|x| \rightarrow \infty$, then $F^{-} \rightarrow 0$ as $|x| \rightarrow \infty$.

By multiplying this equation by $-\mu_{\mathfrak{u}}^{-1} F^{-}$and integrating, we obtain:

$$
\begin{aligned}
\int_{\Omega^{c} \times \mathbb{R}^{3}} \mathrm{~d} x \mathrm{~d} v \mu_{\mathfrak{u}}^{-1} v \cdot \nabla \frac{\left(F^{-}\right)^{2}}{2}=-\varepsilon^{-1} & \int_{\Omega^{c} \times \mathbb{R}^{3}} \mathrm{~d} x \mathrm{~d} v \mathbf{1}_{F^{-} \neq 0} Q^{+}\left(F^{+}, F^{+}\right) F^{-} \mu_{\mathfrak{u}}^{-1} \\
& +\varepsilon^{-1} \int_{\Omega^{c} \times \mathbb{R}^{3}} \mathrm{~d} x \mathrm{~d} v \mathbf{1}_{F^{-} \neq 0} \mu_{\mathfrak{u}}^{-1} F^{-}\left[Q\left(\mu_{\mathfrak{u}}, F^{-}\right)+Q\left(F^{-}, \mu_{\mathfrak{u}}\right)\right]
\end{aligned}
$$

By the spectral inequality,

$$
\begin{aligned}
-\int_{\Omega^{c} \times \mathbb{R}^{3}} \mathrm{~d} x \mathrm{~d} v \mathbf{1}_{F^{-}} \neq 0 & \mu_{\mathfrak{u}}^{-1} F^{-}\left[Q\left(\mu_{\mathfrak{u}}, F^{-}\right)+Q\left(F^{-}, \mu_{\mathfrak{u}}\right)\right] \\
& =-\int_{\Omega^{c} \times \mathbb{R}^{3}} \mathrm{~d} x \mathrm{~d} v \mu_{\mathfrak{u}}^{-1} F^{-}\left[Q\left(\mu_{\mathfrak{u}}, F^{-}\right)+Q\left(F^{-}, \mu_{\mathfrak{u}}\right)\right] \gtrsim\left\|\left(\mathbf{I}-\mathbf{P}_{\mathfrak{u}}\right)\left(\mu_{\mathfrak{u}}^{-\frac{1}{2}} F^{-}\right)\right\|_{2}^{2}
\end{aligned}
$$

Therefore by also integrating by parts the l.h.s., we obtain

$$
\begin{aligned}
& \frac{1}{2} \int_{\partial \Omega \times \mathbb{R}^{3}} \mathrm{~d} S(x) \int_{\mathbb{R}^{3}} \mathrm{~d} v \mu_{\mathfrak{u}}^{-1} v \cdot n\left(F^{-}\right)^{2}+\varepsilon^{-1}\left\|\left(\mathbf{I}-\mathbf{P}_{\mathfrak{u}}\right)\left(\mu_{\mathfrak{u}}^{-\frac{1}{2}} F^{-}\right)\right\|_{2}^{2} \\
& \lesssim-\varepsilon^{-1} \int_{\Omega^{c} \times \mathbb{R}^{3}} \mathrm{~d} x \mathrm{~d} v \mathbf{1}_{F^{-} \neq 0} Q^{+}\left(F^{+}, F^{+}\right) F^{-} \mu_{\mathfrak{u}}^{-1} \leq 0
\end{aligned}
$$


This implies that $F^{-}=0$ on $\gamma^{+}$, thus $F^{-}=0$ on $\gamma$. Moreover $\left(\mathbf{I}-\mathbf{P}_{\mathfrak{u}}\right)\left(\mu_{\mathfrak{u}}^{-\frac{1}{2}} F^{-}\right)=0$ and hence $Q\left(\mu_{\mathfrak{u}}, F^{-}\right)+Q\left(F^{-}, \mu_{\mathfrak{u}}\right)=0$. Thus

$$
-v \cdot \nabla F^{-}=\varepsilon^{-1} \mathbf{1}_{F^{-} \neq 0} Q^{+}\left(F^{+}, F^{+}\right) \geq 0 .
$$

Therefore $F^{-}$satisfies

$$
v \cdot \nabla F^{-} \leq 0 \quad \text { in } \Omega^{c}, \quad F^{-}=0 \quad \text { on } \gamma .
$$

This implies that $F^{-} \leq 0$, but $F^{-} \geq 0$ by definition and hence $F^{-}=0$ identically. Then, $F=F^{+}$ and (6.1.1) coincides with the Boltzmann equation (1.1) and (6.1.2) is the usual diffuse reflection boundary condition (1.8).

Therefore, to construct a positive solution to (1.1) we need to construct a solution to (6.1.1), (6.1.2). We need some notation:

Let $\chi=\mathbf{1}_{|v|<\varepsilon^{-m}}, \bar{\chi}=\mathbf{1}_{|v| \geq \varepsilon^{-m}}=1-\chi$ where $m>0$ is such such that

$$
\mu_{\mathfrak{u}}+\varepsilon \sqrt{\mu}_{\mathfrak{u}} \chi\left(f_{1}+\varepsilon f_{2}\right)>0 .
$$

Such an $m$ certainly exist because, by definition $f_{1}$ and by [5] $f_{2}$, are bounded by $\sqrt{\mu}_{\mathfrak{u}} P_{s}$, for some $s>1$, where $P_{s}$ is a polynomial of degree $s$ in $v$.

Since, for $\beta>0, \exp \left[-\varepsilon^{-\beta}\right] \lesssim \varepsilon^{\ell}$ for any $\ell>0$, in the rest of this section we shall use the short notation

$$
\varepsilon^{\infty}=\exp \left[-\varepsilon^{-\beta}\right], \quad \text { for some } \beta>0 .
$$

Remind that

$$
0 \leq M_{1, \varepsilon(\mathfrak{u}+u), 1}=\mu_{\mathfrak{u}}+\varepsilon \sqrt{\mu_{\mathfrak{u}}} f_{1}+\varepsilon^{2} \sqrt{\mu_{\mathfrak{u}}} \phi_{\varepsilon} .
$$

We denote

$$
\mathscr{Q}=f_{1}+\varepsilon\left(\chi f_{2}+\bar{\chi} \phi_{\varepsilon}\right) .
$$

By (6.1.3), if $\chi=1$, then $\mu_{\mathfrak{u}}+\varepsilon \sqrt{\mu} \mathscr{Q} \geq 0$, and the same is true if $\bar{\chi}=1$ by (6.1.5). Therefore

$$
\mu_{\mathfrak{u}}+\varepsilon \sqrt{\mu} \mathscr{Q} \geq 0 .
$$

We decompose

$$
F=\mu_{\mathfrak{u}}+\varepsilon \sqrt{\mu}_{\mathfrak{u}} \mathscr{Q}+\varepsilon^{\frac{3}{2}} R \sqrt{\mu}_{\mathfrak{u}}
$$

Then we define

$$
\bar{R}=\left\{\begin{array}{l}
R \quad \text { if } \mu_{\mathfrak{u}}+\varepsilon \sqrt{\mu} \mathscr{Q}+\varepsilon^{\frac{3}{2}} \sqrt{\mu_{\mathfrak{u}}} R \geq 0 \\
-\varepsilon^{-\frac{3}{2}}\left(\mu_{\mathfrak{u}}+\varepsilon \mathscr{Q} \sqrt{\mu_{\mathfrak{u}}}\right) \quad \text { if } \mu_{\mathfrak{u}}+\varepsilon \mathscr{Q} \sqrt{\mu_{\mathfrak{u}}}+\varepsilon^{\frac{3}{2}} \sqrt{\mu_{\mathfrak{u}}} R<0
\end{array},\right.
$$

and

$$
\tilde{R}=\bar{R}-R \text {. }
$$

It follows that

$$
\begin{gathered}
F^{+}=\mu_{\mathfrak{u}}+\varepsilon \sqrt{\mu_{\mathfrak{u}}} \mathscr{Q}+\varepsilon^{\frac{3}{2}} \bar{R} \sqrt{\mu_{\mathfrak{u}}} . \\
F^{-}=\varepsilon^{\frac{3}{2}} \tilde{R} \sqrt{\mu}_{\mathfrak{u}} .
\end{gathered}
$$

Indeed, if $F(x, v)>0$, then

$$
F^{+}=F=\mu_{\mathfrak{u}}+\varepsilon \sqrt{\mu}_{\mathfrak{u}} \mathscr{Q}+\varepsilon^{\frac{3}{2}} R \sqrt{\mu}_{\mathfrak{u}}=\mu_{\mathfrak{u}}+\varepsilon \sqrt{\mu} \mathscr{Q}+\varepsilon^{\frac{3}{2}} \bar{R} \sqrt{\mu}_{\mathfrak{u}} .
$$

Moreover, if $F(x, v) \leq 0$, then $\varepsilon^{\frac{3}{2}} R \leq-\left(\mu_{\mathfrak{u}}+\varepsilon \mathscr{Q} \sqrt{\mu_{\mathfrak{u}}}\right)$ and hence

$$
0=\mu_{\mathfrak{u}}+\varepsilon \mathscr{Q} \sqrt{\mu_{\mathfrak{u}}}+\varepsilon^{\frac{3}{2}} \bar{R} \sqrt{\mu}_{\mathfrak{u}}=F^{+},
$$

and

$$
F^{-}=F^{+}-F=\mu_{\mathfrak{u}}+\varepsilon \sqrt{\mu} \mathscr{Q}+\varepsilon^{\frac{3}{2}} \bar{R} \sqrt{\mu}_{\mathfrak{u}}-\left(\mu_{\mathfrak{u}}+\varepsilon \sqrt{\mu} \mathscr{Q}+\varepsilon^{\frac{3}{2}} R \sqrt{\mu}_{\mathfrak{u}}\right)=\varepsilon^{\frac{3}{2}}(\bar{R}-R) \sqrt{\mu}_{\mathfrak{u}}=\varepsilon^{\frac{3}{2}} \tilde{R} \sqrt{\mu}_{\mathfrak{u}} .
$$


Lemma 6.3. We have the following inequalities:

$$
\begin{aligned}
& |\bar{R}| \leq|R|, \\
& |\tilde{R}| \leq \mathbf{1}_{\left\{\mu_{\mathrm{u}}+\varepsilon \sqrt{\mu_{u}} \mathscr{Q}+\varepsilon^{\frac{3}{2}} \bar{R}<0\right\}} 2|R|, \\
& \left|\bar{R}_{1}-\bar{R}_{2}\right| \leq\left|R_{1}-R_{2}\right| \\
& \left|\tilde{R}_{1}-\tilde{R}_{2}\right| \leq 2\left|R_{1}-R_{2}\right|\left(\mathbf{1}_{\left\{\mu_{\mathfrak{u}}+\varepsilon \sqrt{\mu_{\mathfrak{u}}} \mathscr{Q}+\varepsilon^{\frac{3}{2}} R_{1} \sqrt{\left.\mu_{\mathfrak{u}}<0\right\}}\right.}+\mathbf{1}_{\left\{\mu_{\mathfrak{u}}+\varepsilon \sqrt{\mu_{\mathfrak{u}}} \mathscr{Q}+\varepsilon^{\frac{3}{2}} R_{2} \sqrt{\mu_{\mathfrak{u}}}<0\right\}}\right) .
\end{aligned}
$$

Proof. Indeed, $\mu_{\mathfrak{u}}+\varepsilon \mathscr{Q} \sqrt{\mu}_{\mathfrak{u}}+\varepsilon^{\frac{3}{2}} \sqrt{\mu}_{\mathfrak{u}} R<0$ implies $R<0$ and hence $\varepsilon^{\frac{3}{2}}|\bar{R}|=-\varepsilon^{\frac{3}{2}} \bar{R}={\sqrt{\mu_{\mathfrak{u}}}}^{-1}\left(\mu_{\mathfrak{u}}+\right.$ $\left.\varepsilon \sqrt{\mu_{\mathfrak{u}}} \mathscr{Q}\right)<-\varepsilon^{\frac{3}{2}} R=\varepsilon^{\frac{3}{2}}|R|$, which proves (6.1.11). Moreover, $|\tilde{R}| \leq(|R|+|\bar{R}|) \mathbf{1}_{\left\{\mu_{\mathfrak{u}}+\varepsilon \sqrt{\mu}\left(\mathscr{Q}+\varepsilon^{\frac{1}{2}} \bar{R}\right)<0\right\}} \leq$ $2|R| \mathbf{1}_{\left\{\mu_{\mathfrak{u}}+\varepsilon \sqrt{\mu}\left(\mathscr{Q}+\varepsilon^{\frac{1}{2}} \bar{R}\right)<0\right\}}$ which proves (6.1.12). Furthermore given $F_{1}$ and $F_{2}$, we have

$$
\begin{gathered}
\left|F_{1}^{+}-F_{2}^{+}\right| \leq\left|F_{1}-F_{2}\right| . \\
\left|F_{1}^{-}-F_{2}^{-}\right| \leq 2\left|F_{1}-F_{2}\right| .
\end{gathered}
$$

In fact, fixed $(x, v)$, without loss of generality suppose $F_{1}(x, v) \geq F_{2}(x, v)$. If $F_{2}(x, v)>0$ there nothing to show. Thus assume $F_{2}(x, v) \leq 0$. If $F_{1}(x, v) \leq 0$ then $F_{1}^{+}(x, v)=F_{2}^{+}(x, v)=0$ and the inequality is obviously verified. Therefore we only need to consider the case $F_{1}(x, v)>0$ and $F_{2} \leq 0$. We have

$$
\left|F_{1}^{+}(x, v)-F_{2}^{+}(x, v)\right|=F_{1}(x, v) \leq F_{1}(x, v)-F_{2}(x, v)=\left|F_{1}(x, v)-F_{2}(x, v)\right| .
$$

Moreover, since $F^{-}=F^{+}-F,\left|F_{1}^{-}-F_{2}^{-}\right| \leq\left|F_{1}^{+}-F_{2}^{+}\right|+\left|F_{1}-F_{2}\right| \leq 2\left|F_{1}-F_{2}\right|$. Therefore, with $R_{i}$ defined by (6.1.6) and $\bar{R}_{i}$ by (6.1.9), it follows that

$$
\begin{gathered}
\varepsilon^{\frac{3}{2}}\left|\bar{R}_{1}-\bar{R}_{2}\right|=\sqrt{\mu}_{\mathfrak{u}}^{-1}\left|\left(F_{1}^{+}-\mu_{\mathfrak{u}}-\varepsilon \sqrt{\mu_{\mathfrak{u}}} \mathscr{Q}\right)-\left(F_{2}^{+}-\mu_{\mathfrak{u}}-\varepsilon \sqrt{\mu_{\mathfrak{u}}} \mathscr{Q}\right)\right|=\sqrt{\mu}_{\mathfrak{u}}^{-1}\left|F_{1}^{+}-F_{2}^{+}\right| \leq \sqrt{\mu}_{\mathfrak{u}}^{-1}\left|F_{1}-F_{2}\right| \\
=\sqrt{\mu}_{\mathfrak{u}}^{-1}\left|\left(F_{1}-\mu_{\mathfrak{u}}-\varepsilon \sqrt{\mu_{\mathfrak{u}}} \mathscr{Q}\right)-\left(F_{2}-\mu_{\mathfrak{u}}-\varepsilon \sqrt{\mu_{\mathfrak{u}}} \mathscr{Q}\right)\right|=\varepsilon^{\frac{3}{2}}\left|R_{1}-R_{2}\right| .
\end{gathered}
$$

Hence (6.1.13) is proved. Furthermore

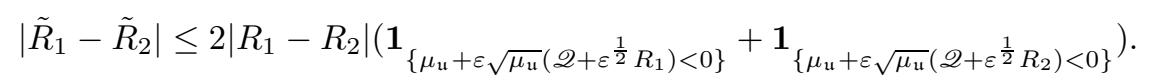

In fact, since $F_{1}^{-}-F_{2}^{-}$vanishes outside of the set

$$
\left\{\mu_{\mathfrak{u}}+\varepsilon \sqrt{\mu_{\mathfrak{u}}}\left(\mathscr{Q}+\varepsilon^{\frac{1}{2}} R_{1}\right)<0\right\} \cup\left\{\mu_{\mathfrak{u}}+\varepsilon \sqrt{\mu_{\mathfrak{u}}}\left(\mathscr{Q}+\varepsilon^{\frac{1}{2}} R_{2}\right)<0\right\}
$$

and $\varepsilon^{-\frac{3}{2}}\left|F_{1}-F_{2}\right|=\left|R_{1}-R_{2}\right|$, we have

$$
\begin{aligned}
& \left|\tilde{R}_{1}-\tilde{R}_{2}\right|=\varepsilon^{-\frac{3}{2}} \sqrt{\mu_{\mathfrak{u}}^{-1}} \mid\left(F_{1}^{-}-\mu_{\mathfrak{u}}-\varepsilon \sqrt{\mu_{\mathfrak{u}}} \mathscr{Q}\right)-\left(F_{2}^{-}-\left(\mu_{\mathfrak{u}}-\varepsilon \sqrt{\mu_{\mathfrak{u}}} \mathscr{Q}\right)\left|=\varepsilon^{-\frac{3}{2}} \sqrt{\mu_{\mathfrak{u}}^{-1}}\right| F_{1}^{-}-F_{2}^{-} \mid \leq\right. \\
& 2 \varepsilon^{-\frac{3}{2}} \sqrt{\mu_{\mathfrak{u}}^{-1}}\left|F_{1}-F_{2}\right|\left(\mathbf{1}_{\left\{\mu_{\mathfrak{u}}+\varepsilon \sqrt{\mu_{\mathfrak{u}}}\left(\mathscr{Q}+\varepsilon^{\frac{1}{2}} R_{1}\right)<0\right\}}+\mathbf{1}_{\left\{\mu_{\mathfrak{u}}+\varepsilon \sqrt{\mu_{\mathfrak{u}}}\left(\mathscr{Q}+\varepsilon^{\frac{1}{2}} R_{2}\right)<0\right\}}\right)=
\end{aligned}
$$

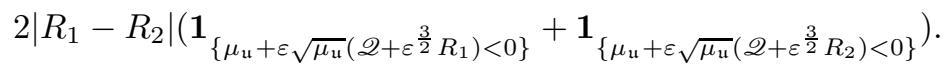

As for the boundary conditions, we have

$$
\mu_{\mathfrak{u}}+\varepsilon f_{1} \mu_{\mathfrak{u}}^{\frac{1}{2}}+\varepsilon^{2}\left(\chi f_{2}+\bar{\chi} \phi_{\varepsilon}\right) \mu_{\mathfrak{u}}^{\frac{1}{2}}+\varepsilon^{\frac{3}{2}} R \mu_{\mathfrak{u}}^{\frac{1}{2}}=\mathcal{P}_{\gamma}^{w}\left[\mu_{\mathfrak{u}}+\varepsilon \chi f_{1} \mu_{\mathfrak{u}}^{\frac{1}{2}}+\varepsilon^{2}\left(\chi f_{2}+\bar{\chi} \phi_{\varepsilon}\right) \mu_{\mathfrak{u}}^{\frac{1}{2}}+\varepsilon^{\frac{3}{2}} \bar{R} \mu_{\mathfrak{u}}^{\frac{1}{2}}\right] .
$$

Therefore, subtracting this equations from (1.33),

$$
\varepsilon^{2} \chi\left(f_{2}-\phi_{\varepsilon}\right) \mu_{\mathfrak{u}}^{\frac{1}{2}}+\varepsilon^{\frac{3}{2}} R \mu_{\mathfrak{u}}^{\frac{1}{2}}=\mathcal{P}_{\gamma}^{w}\left[\varepsilon^{2} \chi\left(f_{2}-\phi_{\varepsilon}\right) \mu_{\mathfrak{u}}^{\frac{1}{2}}+\varepsilon^{\frac{3}{2}} \bar{R} \mu_{\mathfrak{u}}^{\frac{1}{2}}\right] .
$$

Hence

with

$$
R=P_{\gamma}^{\mathfrak{u}} R+\varepsilon^{\frac{1}{2}} \bar{r}+P_{\gamma}^{\mathfrak{u}} \tilde{R}
$$

We have

$$
\bar{r}=P_{\gamma}^{\mathfrak{u}}\left[\chi\left(f_{2}-\phi_{\varepsilon}\right)\right]-\chi\left(f_{2}-\phi_{\varepsilon}\right) .
$$

$$
\left\|\int_{\mathbb{R}^{3}} d v\left[\mu_{\mathfrak{u}}+\sqrt{\mu_{\mathfrak{u}}} \varepsilon \mathscr{Q}\right] v \cdot n\right\|_{\infty}=\varepsilon^{\infty} \quad \text { on } \partial \Omega .
$$


In fact

$$
\int_{\mathbb{R}^{3}} \mathrm{~d} v\left(\mu_{\mathfrak{u}}+\left(\varepsilon f_{1}+\varepsilon^{2} \phi_{\varepsilon}\right) \sqrt{\mu_{\mathfrak{u}}}\right) v \cdot n=\int_{\mathbb{R}^{3}} \mathrm{~d} v M_{1, \varepsilon(\mathfrak{u}+u), 1} v \cdot n=\varepsilon n \cdot(\mathfrak{u}+u)=0,
$$

on $\partial \Omega$ because $u=-\mathfrak{u}$ on $\partial \Omega$, see (1.14). We have also $\int_{\mathbb{R}^{3}} d v\left(\mu_{\mathfrak{u}}+\varepsilon \sqrt{\mu}_{\mathfrak{u}} f_{1}\right) v \cdot n=0$ on $\partial \Omega$ and hence $\int_{\mathbb{R}^{3}} d v \sqrt{\mu}_{\mathfrak{u}} \phi_{\varepsilon} v \cdot n=0$ on $\partial \Omega$. Therefore, by (1.31), since $\left.u\right|_{\partial \Omega}=-\mathfrak{u}$,

$$
\left|\int_{\mathbb{R}^{3}} \mathrm{~d} v n \cdot v \sqrt{\mu_{\mathfrak{u}}} \chi \phi_{\varepsilon}\right|=\left|-\int_{\mathbb{R}^{3}} \mathrm{~d} v n \cdot v \sqrt{\mu_{\mathfrak{u}}} \bar{\chi} \phi_{\varepsilon}\right| \leq e^{-\varepsilon^{-m}}|\mathfrak{u}|^{2} \lesssim \varepsilon^{\infty} \quad \text { on } \partial \Omega .
$$

Since $\mathbf{P}_{\mathfrak{u}} f_{2}=0$, in the same way we obtain

$$
\left|\int_{\mathbb{R}^{3}} \mathrm{~d} v n \cdot v \sqrt{\mu_{\mathfrak{u}}} \chi f_{2}\right|=\left|-\int_{\mathbb{R}^{3}} \mathrm{~d} v n \cdot v \sqrt{\mu_{\mathfrak{u}}} \bar{\chi} f_{2}\right| \leq e^{-\varepsilon^{-m}}|\mathfrak{u}|^{2} \lesssim \varepsilon^{\infty} \quad \text { on } \partial \Omega,
$$

because $\left|f_{2}\right| \leq \sqrt{\mu}_{\mathfrak{u}} P_{\ell} \mid\left(|\nabla u|+|u|^{2}\right)$ and $\nabla u$ is bounded in $L^{p}$ for any $p>\frac{4}{3}$ and (6.1.17) follows.

The boundary conditions for $F$ imply

$$
\int_{\mathbb{R}^{3}} F \mathrm{~d} v v \cdot n=-\int_{\{v \cdot n>0\}} \mathrm{d} v F^{-} n \cdot v
$$

on $\partial \Omega$. Therefore we have

$$
\int_{\mathbb{R}^{3}} \mathrm{~d} v \sqrt{\mu}_{\mathfrak{u}} R v \cdot n=-\int_{v \cdot n>0} \mathrm{~d} v \sqrt{\mu_{\mathfrak{u}}} \tilde{R} n \cdot v+O\left(\varepsilon^{\infty}\right)
$$

Lemma 6.4.

$$
\left\|\int_{v \cdot n<0} \mathrm{~d} v \bar{r} \sqrt{\mu_{\mathfrak{u}}} n \cdot v\right\|_{\infty} \lesssim \varepsilon^{\infty}
$$

Proof. We have $\bar{r}=P_{\gamma}^{\mathfrak{u}}\left(\chi f_{2}-\bar{\chi} \phi_{\varepsilon}\right)-\left(\chi f_{2}-\bar{\chi} \phi_{\varepsilon}\right)$ and, using (5.3.10), $\int_{v \cdot n<0} \mathrm{~d} v \bar{r} \sqrt{\mu_{\mathfrak{u}}} n \cdot v=$ $\int_{\mathbb{R}^{3}} \mathrm{~d} v \sqrt{\mu_{\mathfrak{u}}}\left(\chi f_{2}-\chi \phi_{\varepsilon}\right) n \cdot v$. (6.1.19) and (6.1.20) imply (6.1.21).

We rewrite the problem (6.1.1), 6.1.2) using the decompositions (6.1.6) and (6.1.9). Reminding the definitions of $f_{1}, f_{2}$ and the incompressible Navier-Stokes equations, we are reduced to construct the solution to the problem:

$$
\begin{aligned}
& v \cdot \nabla R+\varepsilon^{-1} L_{\mathfrak{u}} R=L_{\mathfrak{u}}^{(1)} \bar{R}+\varepsilon^{\frac{1}{2}} \Gamma_{\mathfrak{u}}(\bar{R}, \bar{R})+\varepsilon^{\frac{1}{2}} \bar{A}_{\mathfrak{u}}, \\
& \left.R\right|_{\gamma_{-}}=P_{\gamma}^{\mathfrak{u}} R+\varepsilon^{\frac{1}{2}} r, \\
& \text { where } \\
& L_{\mathfrak{u}}^{(1)} \bar{R}=2 \tilde{\Gamma}_{\mathfrak{u}}(\mathscr{Q}, \bar{R}), \\
& \mathbf{P}_{\mathfrak{u}} \bar{A}_{\mathfrak{u}}=\mathbf{P}_{\mathfrak{u}}\left[\bar{\chi} v \cdot \nabla\left(\bar{\chi}\left(\phi_{\varepsilon}-f_{2}\right)\right)-\varepsilon \mathfrak{u} \cdot \nabla f_{2}\right] \\
& \left(\mathbf{I}-\mathbf{P}_{\mathfrak{u}}\right) \bar{A}_{\mathfrak{u}}=\left(\mathbf{I}-\mathbf{P}_{\mathfrak{u}}\right)\left(v \cdot \nabla\left(\chi f_{2}+\bar{\chi} \phi_{\varepsilon}\right)\right) \\
& -\tilde{\Gamma}_{\mathfrak{u}}\left(2 f_{1}+\varepsilon\left(\chi f_{2}+\bar{\chi} \phi_{\varepsilon}\right), \chi f_{2}+\bar{\chi} \phi_{\varepsilon}\right)+\varepsilon^{-1} L_{\mathfrak{u}}\left[\bar{\chi}\left(\phi_{\varepsilon}-f_{2}\right)\right], \\
& r=\bar{r}-\varepsilon^{-\frac{1}{2}} P_{\gamma}^{\mathfrak{u}} \tilde{R} .
\end{aligned}
$$

In fact, reminding (1.21) and (1.20), we have

$$
L_{\mathfrak{u}}\left(\chi f_{2}\right)-\Gamma_{\mathfrak{u}}\left(f_{1}, f_{1}\right)+\left(\mathbf{I}-\mathbf{P}_{\mathfrak{u}}\right)\left(v \cdot \nabla f_{1}\right)=-L_{\mathfrak{u}}\left(\bar{\chi} f_{2}\right),
$$

and

$$
\mathbf{P}_{\mathfrak{u}}\left(v \cdot \nabla \chi f_{2}\right)=-\mathbf{P}_{\mathfrak{u}}\left(v \cdot \nabla \bar{\chi} f_{2}\right),
$$

so that

$$
v \cdot \nabla\left(\chi f_{2}\right)=\left(\mathbf{I}-\mathbf{P}_{\mathfrak{u}}\right)\left(v \cdot \nabla\left(\chi f_{2}\right)\right)-\mathbf{P}_{\mathfrak{u}}\left(v \cdot \nabla\left(\bar{\chi} f_{2}\right)\right)
$$


Therefore,

$$
\begin{aligned}
\bar{A}_{\mathfrak{u}}: & =\varepsilon^{-\left(\frac{1}{2}+\frac{3}{2}\right)}\left\{\varepsilon\left\{\left(\mathbf{I}-\mathbf{P}_{\mathfrak{u}}\right)\left(v \cdot \nabla f_{1}\right)+L_{\mathfrak{u}}\left(\chi f_{2}\right)-\Gamma_{\mathfrak{u}}\left(f_{1}, f_{1}\right)\right\}+\varepsilon L_{\mathfrak{u}}\left(\bar{\chi} \phi_{\varepsilon}\right)\right. \\
& \left.+\varepsilon^{2} v \cdot \nabla\left(\chi f_{2}+\bar{\chi} \phi_{\varepsilon}\right)-\varepsilon^{2} \tilde{\Gamma}_{\mathfrak{u}}\left(\left(\chi f_{2}+\bar{\chi} \phi_{\varepsilon}\right), 2 f_{1}+\varepsilon\left(\chi f_{2}+\bar{\chi} \phi_{\varepsilon}\right)\right)\right\}-\varepsilon \mathfrak{u} \cdot \nabla f_{2} \\
& =-\varepsilon^{-1} L_{\mathfrak{u}}\left(\bar{\chi} f_{2}\right)+\varepsilon^{-1} L_{\mathfrak{u}}\left(\bar{\chi} \phi_{\varepsilon}\right)+\left(\mathbf{I}-\mathbf{P}_{\mathfrak{u}}\right)\left(v \cdot \nabla\left(\chi f_{2}\right)\right) \\
& -\mathbf{P}_{\mathfrak{u}}\left(v \cdot \nabla\left(\bar{\chi} f_{2}\right)\right)+v \cdot \nabla\left(\bar{\chi} \phi_{\varepsilon}\right)-\tilde{\Gamma}_{\mathfrak{u}}\left(\left(\chi f_{2}+\bar{\chi} \phi_{\varepsilon}\right), 2 f_{1}+\varepsilon\left(\chi f_{2}+\bar{\chi} \phi_{\varepsilon}\right)\right)-\varepsilon \mathfrak{u} \cdot \nabla f_{2} \\
& =\varepsilon^{-1} L_{\mathfrak{u}}\left[\bar{\chi}\left(\phi_{\varepsilon}-f_{2}\right)\right]+\mathbf{P}_{\mathfrak{u}}\left[v \cdot \nabla\left(\bar{\chi}\left(\phi_{\varepsilon}-f_{2}\right)\right)\right]+\left(\mathbf{I}-\mathbf{P}_{\mathfrak{u}}\right)\left(v \cdot \nabla\left(\chi f_{2}+\chi_{\mathfrak{u}} \phi_{\varepsilon}\right)\right) \\
& -\tilde{\Gamma}_{\mathfrak{u}}\left(\left(\chi f_{2}+\bar{\chi} \phi_{\varepsilon}\right), 2 f_{1}+\varepsilon\left(\chi f_{2}+\bar{\chi} \phi_{\varepsilon}\right)\right)-\varepsilon \mathfrak{u} \cdot \nabla f_{2} .
\end{aligned}
$$

Proposition 6.5. Let $X \in L^{p}\left(\Omega^{c} \times \mathbb{R}^{3}\right)$ and $\bar{X}$ and $\tilde{X}$ be defined as $\bar{X}$ and $\tilde{R}$, as in (6.1.7) and (6.1.8). Assume $p>1,|\mathfrak{u}| \ll 1$, Then:

(1) Let $w(v)$ be such that $w^{-1} \lesssim \sqrt{\mu_{\mathfrak{u}}^{\beta}}\langle v\rangle^{-\beta^{\prime}}$ for some $0<\beta \ll 1$ and $\beta^{\prime}>0$. If $\varepsilon^{\frac{1}{2}}\|w X\|_{\infty}$ is bounded, then

$$
\begin{aligned}
\left|P_{\gamma}^{\mathfrak{u}} \tilde{X}\right|_{2,-} & \lesssim\left[\varepsilon\left(|\mathfrak{u}|+\varepsilon^{\frac{1}{2}}\|w X\|_{\infty}\right)\right]^{1+\beta}|X|_{2,+} . \\
\left|P_{\gamma}^{\mathfrak{u}} \tilde{X}\right|_{\infty,-} & \lesssim\left[\varepsilon\left(|\mathfrak{u}|+\varepsilon^{\frac{1}{2}}\|w X\|_{\infty}\right)\right]^{1+\beta}|X|_{\infty,+} .
\end{aligned}
$$

(2) Given $X_{1}$ and $X_{2}$ such that $\varepsilon^{\frac{1}{2}}\left\|w X_{i}\right\|_{\infty}$ are bounded, then,

$$
\begin{aligned}
& \left|P_{\gamma}^{\mathfrak{u}}\left[\tilde{X}_{1}-\tilde{X}_{2}\right]\right|_{2,-} \lesssim\left[\varepsilon\left(|\mathfrak{u}|+\max _{i=1,2}\left(\varepsilon^{\frac{1}{2}}\left\|w X_{i}\right\|_{\infty}\right)\right)\right]^{1+\beta}\left|X_{1}-X_{2}\right|_{2,+} . \\
& \left|P_{\gamma}^{\mathfrak{u}}\left[\tilde{X}_{1}-\tilde{X}_{2}\right]\right|_{\infty,-} \lesssim\left[\varepsilon\left(|\mathfrak{u}|+\max _{i=1,2}\left(\varepsilon^{\frac{1}{2}}\left\|w X_{i}\right\|_{\infty}\right)\right)\right]^{1+\beta}\left|X_{1}-X_{2}\right|_{\infty} .
\end{aligned}
$$

(3) $\Gamma_{\mathfrak{u}}^{ \pm}(\bar{X}, \bar{X}) \leq \Gamma_{\mathfrak{u}}^{ \pm}(|X|,|X|) \mid$ and $\left|\Gamma_{\mathfrak{u}}^{ \pm}\left(\bar{X}_{1}, \bar{X}_{1}\right)-\tilde{\Gamma}_{\mathfrak{u}}^{ \pm}\left(\bar{X}_{2}, \bar{X}_{2}\right)\right| \lesssim \tilde{\Gamma}_{\mathfrak{u}}^{ \pm}\left(\left|X_{1}\right|+\left|X_{2}\right|,\left|X_{1}-X_{2}\right|\right)$.

Proof. To prove (6.1.28), note that

$$
\mathbf{1}_{\left\{\mu_{\mathfrak{u}}+\varepsilon \sqrt{\mu_{\mathfrak{u}}}\left\{\mathscr{Q}+\varepsilon^{\frac{1}{2}} X\right\}<0\right\}} \leq \mathbf{1}_{\left\{\sqrt{\mu_{\mathfrak{u}}}<\varepsilon\left(|\mathscr{Q}|+\varepsilon^{\frac{1}{2}}\|w X\|_{\infty}\right) w^{-1}\right\}}
$$

Therefore, by (6.1.12), since $w^{-1} \lesssim \sqrt{\mu}_{\mathfrak{u}}^{\beta}\langle v\rangle^{-\beta^{\prime}}$ for some $0<\beta \ll 1$ and $\beta^{\prime}>0$, and $|\mathscr{Q}| \lesssim$ $|\mathfrak{u}| \sqrt{\mu}_{\mathfrak{u}}\langle v\rangle^{\ell}$ for some $\ell>0$, we have

$$
\begin{aligned}
& \left|P_{\gamma}^{\mathfrak{u}} \tilde{X}\right| \leq 2 \frac{\mu}{\sqrt{\mu_{\mathfrak{u}}(v)}} \int_{v^{\prime} \cdot n>0} \mathrm{~d} v^{\prime} \sqrt{\mu_{\mathfrak{u}}\left(v^{\prime}\right)}\left|v^{\prime} \cdot n\right| \mathbf{1}_{\left\{\sqrt{\mu_{\mathfrak{u}}\left(v^{\prime}\right)}<\varepsilon\left(|\mathscr{Q}|+\varepsilon^{\frac{1}{2}}\|w X\|_{\infty} w^{-1}\right)\right\}}|X| d v^{\prime} \\
& \leq 2\left\|X|v \cdot n|^{\frac{1}{2}}\right\|_{L_{v}^{2}} \frac{\mu}{\sqrt{\mu_{\mathfrak{u}}(v)}}\left(\int_{\mathbb{R}^{3}} \mathrm{~d} v^{\prime} w^{-2}\left(v^{\prime}\right) \mathbf{1}_{\left\{\sqrt{\mu_{\mathfrak{u}}\left(v^{\prime}\right)}<\varepsilon\left(|\mathscr{Q}|+\varepsilon^{\frac{1}{2}}\|w X\|_{\infty} w^{-1}\right)\right\}} \mu_{\mathfrak{u}}\left(v^{\prime}\right)\left|v^{\prime} \cdot n\right|\right)^{\frac{1}{2}} \\
& \quad \lesssim\left[\varepsilon\left(|\mathfrak{u}|+\varepsilon^{\frac{1}{2}}\|w X\|_{\infty}\right)\right]^{1+\beta}\left\|X|v \cdot n|^{\frac{1}{2}}\right\|_{L_{v}^{2}} \frac{\mu}{\sqrt{\mu_{\mathfrak{u}}(v)}}, \quad, 6.1
\end{aligned}
$$

because $\int_{\mathbb{R}^{3}} d v^{\prime}\langle v\rangle^{-2 \beta^{\prime}}\left(v^{\prime}\right)\left|v^{\prime} \cdot n\right| \lesssim 1$ by choosing $\beta^{\prime}>2$. Therefore

$$
\int_{\gamma_{-}} \mathrm{d} v\left|P_{\gamma} \tilde{X}\right|^{2} \lesssim\left[\varepsilon\left(|\mathfrak{u}|+\varepsilon^{\frac{1}{2}}\|w X\|_{\infty}\right)\right]^{2+2 \beta}|X|_{2,+}^{2},
$$

so 6.1 .28 is proven.

We also have

$$
\begin{aligned}
\left|P_{\gamma}^{\mathfrak{u}} \tilde{X}\right| \leq\|X\|_{\infty} \frac{\mu}{\sqrt{\mu_{\mathfrak{u}}(v)}}\left(\int_{\mathbb{R}^{3}} \mathrm{~d} v^{\prime} w^{-2}\left(v^{\prime}\right) \mathbf{1}_{\left\{\sqrt{\left.\mu_{\mathfrak{u}}\left(v^{\prime}\right)<\varepsilon\left(|\mathscr{Q}|+\alpha w^{-1}\right)\right\}}\right.} \mu_{\mathfrak{u}}\left(v^{\prime}\right)\left|v^{\prime} \cdot n\right|^{2}\right)^{\frac{1}{2}} \\
\lesssim\left[\varepsilon\left(|\mathfrak{u}|+\varepsilon^{\frac{1}{2}}\|w X\|_{\infty}\right)\right]^{1+\beta}\|X\|_{\infty} \frac{\mu}{\sqrt{\mu_{\mathfrak{u}}(v)}},
\end{aligned}
$$

from which 6.1.29) follows.

To prove (2), we observe that, if $\left\|w X_{i}\right\|_{\infty} \leq \alpha$, by 6.1.14

$$
\left|P_{\gamma}^{\mathfrak{u}}\left(\tilde{X}_{1}-\tilde{X}_{2}\right)\right| \leq 4 \alpha \frac{\mu}{\sqrt{\mu_{\mathfrak{u}}(v)}} \int_{v^{\prime} \cdot n>0} \mathrm{~d} v^{\prime} \sqrt{\mu_{\mathfrak{u}}\left(v^{\prime}\right)} v^{\prime} \cdot n\left|X_{1}-X_{2}\right| \mathbf{1}_{\left.\sqrt{\mu_{\mathfrak{u}}\left(v^{\prime}\right)}<4 \varepsilon \alpha w^{-1}\right\}} d v^{\prime} .
$$


The rest of the proof is as before.

Statement (3) follows immediately from (6.1.11) and (6.1.13).

Proposition 6.6. Let $u$ be the solution to the incompressible Navier-Stokes equations. Then, if $\varepsilon \ll 1$,

- for any $p>1$

- for any $p>\frac{4}{3}$

$$
\left\|\mathbf{P}_{\mathfrak{u}} \bar{A}_{\mathfrak{u}}\right\|_{p} \lesssim \varepsilon^{\infty}
$$

$$
\begin{aligned}
&\left\|\nu^{-\frac{1}{2}} L_{\mathfrak{u}}^{(1)} \bar{X}\right\|_{p} \lesssim|\mathfrak{u}|\|X\|_{\frac{3 p}{3-p}} \text { for } p<3, \\
&\left\|\nu^{-\frac{1}{2}} L_{\mathfrak{u}}^{(1)} \bar{X}\right\|_{p} \lesssim|\mathfrak{u}|\|w X\|_{\infty} \quad \text { for } p \geq 3 .
\end{aligned}
$$

Proof. First note that, by (6.1.25), since $f_{1}=\sqrt{\mu}_{\mathfrak{u}} v_{\mathfrak{u}} \cdot u$ and $f_{2}=\sum_{i, j=1}^{3} \mathscr{B}_{i, j} \partial_{i} u_{j}+L_{\mathfrak{u}}^{-1} \Gamma_{\mathfrak{u}}\left(f_{1}, f_{1}\right)$, we obtain

$$
\begin{array}{r}
\mathbf{P}_{\mathfrak{u}} \bar{A}_{\mathfrak{u}}=\mathbf{P}_{\mathfrak{u}}\left\{\bar{\chi} v_{\mathfrak{u}} \cdot \nabla \phi_{\varepsilon}+\bar{\chi} \sum_{j_{1}, j_{2}, j_{3}=1}^{3} u_{j_{2}} \partial_{j_{1}} u_{j_{3}} v_{j_{1}} L_{\mathfrak{u}}^{-1} \Gamma_{\mathfrak{u}}\left(v_{\mathfrak{u}, j_{2}} \sqrt{\mu}, v_{\mathfrak{u}, j_{3}} \sqrt{\mu_{\mathfrak{u}}}\right)\right. \\
\left.+\bar{\chi}\left[\sqrt{\mu}_{\mathfrak{u}} \sum_{j_{1}, j_{2}, j_{3}=1}^{3} v_{j_{1}} \mathscr{B}_{j_{2} . j_{3}} \partial_{j_{1}} \partial_{j_{2}} u_{j_{3}}\right]\right\} .
\end{array}
$$

We remind that from [13, Th. X.6.4, we know that, if $\mathfrak{u} \neq 0$, then $u \in L^{p}$ for any $p>2$, $D u \in L^{p}$ for any $p>4 / 3$ and $D^{2} u \in L^{p}$ for any $p>1$. Therefore, for any $p \geq 1,\|u D u\|_{p} \lesssim 1$. Moreover, for any $\beta>0$,

$$
\bar{\chi} \mu_{\mathfrak{u}}^{\beta} \leq \exp \left[-\frac{\beta}{2} \varepsilon^{-2 m}\right] \lesssim \varepsilon^{\infty},
$$

and we obtain that the second term is less than $\varepsilon^{\infty}$ in $L^{p}$-norm, for any $p \geq 1$. From the definition of $\phi_{\varepsilon}$ we have $D \phi_{\varepsilon} \sim \mu_{\mathfrak{u}}^{\frac{1}{2}}|u||D u|$ and hence also the first term is less than $\varepsilon^{\infty}$ in $L^{p}$-norm, for any $p \geq 1$. Finally, since $\left\|D^{2} u\right\|_{p} \lesssim 1$ for any $p>1$, the third term is less than $\varepsilon^{\infty}$ in $L^{p}$-norm, for any $p>1$, so the first item of Proposition 6.6 is proved.

To prove the second item we first observe that, for any $p>1,\left\|\Gamma_{\mathfrak{u}}\left(\chi f_{2}+\bar{\chi} \phi_{\varepsilon}, f_{j}\right)\right\|_{p} \lesssim 1$. This follows as the estimate of $\left\|\Gamma_{\mathfrak{u}}\left(f_{1}, f_{1}\right)\right\|_{p}$. Next we need to take care of the term $\varepsilon^{-1} L \bar{\chi}\left(\mathbf{I}-\mathbf{P}_{\mathfrak{u}}\right)(v$. $\nabla f_{1}$ ) entering in $f_{2}$. Since this is proportional to $D u$ this is bounded in $L^{p}$ for $p>\frac{4}{3}$. The diverging factor $\varepsilon^{-1}$ is dealt with using (6.1.41).

To prove third item we remind that $\|u\|_{3} \lesssim|\mathfrak{u}|$ for $|\mathfrak{u}| \ll 1$ (proof in Appendix) and hence also $\left\|f_{1}\right\|_{3} \lesssim|\mathfrak{u}|$. We use the definition of $L_{\mathfrak{u}}^{(1)}$, the inequalities (6.1.11) and for any $p \geq 1$ and $q^{-1}+q^{-1}=1,\left\|\nu^{-\frac{1}{2}} \Gamma_{\mathfrak{u}}(f, g)\right\|_{p} \leq\left\|\nu^{\frac{1}{2}} f\right\|_{p q}\|g\|_{p q^{\prime}}$ with $q$ such that $p q=3$ and hence $p q^{\prime}=\frac{3 p}{3-p}$ to conclude.

6.2. Iteration. The construction of the solution is obtained as follows: we define the sequence $\{R\}_{\ell=0}^{\infty}$ as: $R_{0}=0 ; R_{\ell+1}$ is the solution to the linear problem

$$
v \cdot \nabla R_{\ell+1}+\varepsilon^{-1} L_{\mathfrak{u}} R_{\ell+1}=L_{\mathfrak{u}}^{(1)} \bar{R}_{\ell}+\varepsilon^{\frac{1}{2}} \Gamma_{\mathfrak{u}}\left(\bar{R}_{\ell}, \bar{R}_{\ell}\right)+\varepsilon^{\frac{1}{2}} \bar{A}_{\mathfrak{u}}
$$

with boundary conditions

where

$$
R_{\ell+1}=P_{\gamma}^{\mathfrak{u}} R_{\ell+1}+\varepsilon^{\frac{1}{2}} r_{\ell}
$$

$$
r_{\ell}=\bar{r}-\varepsilon^{-\frac{1}{2}} P_{\gamma}^{\mathfrak{u}} \tilde{R}_{\ell}
$$

By denoting $\bar{g}=L_{\mathfrak{u}}^{(1)} \bar{R}_{\ell}+\varepsilon^{\frac{1}{2}} \Gamma_{\mathfrak{u}}\left(\bar{R}_{\ell}, \bar{R}_{\ell}\right)+\varepsilon^{\frac{1}{2}} \bar{A}_{\mathfrak{u}}$, we are reduced to the linear problem studied in the previous sections.

Remind the definition (1.38) of $\left[[\cdot]_{\beta, \beta^{\prime}}\right.$. Since in the rest of this section $\beta$ and $\beta^{\prime}$ are fixed, we drop the indices. Let $\mathscr{X}$ be the Banach space of the functions $X(x, v)$ such that $[[X]]$ is finite. 
Theorem 6.7. There are $\vartheta<1$ and $c_{0} \ll 1$ such that, if $\varepsilon \ll 1$ and $|\mathfrak{u}| \leq c_{0} \vartheta$, and

$$
\sup _{0 \leq j \leq \ell}\left[\left[R_{j}\right]\right] \leq \vartheta
$$

then

$$
\left[\left[R_{\ell+1}\right]\right]<\vartheta
$$

Moreover, there is $\lambda<1$ such that

$$
\left[\left[R_{\ell+1}-R_{\ell}\right]\right] \leq \lambda\left[\left[R_{\ell}-R_{\ell-1}\right]\right] .
$$

Therefore $R_{\ell}$ converges [[.]]-strongly to $R \in \mathscr{X}$ which solves (6.1.22), (6.1.23).

Proof. By Theorem 1.5. we need to show that, when $g=\varepsilon^{\frac{1}{2}} \Gamma_{\mathfrak{u}}\left(\bar{R}_{\ell}, \bar{R}_{\ell}\right)+L_{\mathfrak{u}}^{(1)} \bar{R}_{\ell}+\varepsilon^{\frac{1}{2}} \bar{A}_{\mathfrak{u}}$ and $r=\bar{r}+\varepsilon^{-\frac{1}{2}} P_{\gamma}^{\mathfrak{u}} \tilde{R}_{\ell}$, if $\varepsilon \ll 1,|\mathfrak{u}| \ll 1$, then $\mathscr{M}(g, r)<\vartheta$.

We need to bound all the term in the right hand side of (1.44). To estimate the norms of $\Gamma_{\mathfrak{u}}(f, h)$ we state the following

Proposition 6.8. We have the following estimates: let $X \in \mathscr{X}$. Then

$$
\begin{aligned}
& \varepsilon^{\frac{1}{2}}\left\|\nu^{-\frac{1}{2}} \Gamma_{\mathfrak{u}}(\bar{X}, \bar{X})\right\|_{2} \lesssim[[X]]^{2}, \\
& \varepsilon^{\frac{1}{2}}\left\|\nu^{-\frac{1}{2}} w \Gamma_{\mathfrak{u}}(\bar{X}, \bar{X})\right\|_{\infty} \lesssim \varepsilon^{-\frac{1}{2}}[[X]]^{2}, \\
& \varepsilon^{\frac{1}{2}}\left\|\nu^{-\frac{1}{2}} \Gamma_{\mathfrak{u}}(\bar{X}, \bar{X})\right\|_{\frac{3}{2}} \leq \varepsilon^{-\frac{1}{2}}[[X]]^{2} .
\end{aligned}
$$

Proof. We make use of the following inequality (see [10]):

$$
\left\|\nu^{-\frac{1}{2}} \Gamma^{ \pm}(f, h)\right\|_{\frac{q p}{q+p}} \lesssim\left\|\nu^{\frac{1}{2}} f\right\|_{q}\|h\|_{p}
$$

In particular, for $q=3, p=3$ we get

$$
\left\|\nu^{-\frac{1}{2}} \Gamma_{\mathfrak{u}}^{ \pm}(f, h)\right\|_{\frac{3}{2}} \leq\left\|\nu^{\frac{1}{2}} f\right\|_{3}\|h\|_{3},
$$

and for $q=3, p=6$,

$$
\left\|\nu^{-\frac{1}{2}} \Gamma_{\mathfrak{u}}^{ \pm}(f, h)\right\|_{2} \leq\left\|\nu^{\frac{1}{2}} f\right\|_{3}\|h\|_{6} .
$$

We will also use

$$
\left\|\nu^{-\frac{1}{2}} \Gamma^{ \pm}(f, h)\right\|_{2} \lesssim\|f\|_{\nu}\|h\|_{\infty}
$$

and

By (6.1.11),

$$
\left\|\langle v\rangle^{-1} \Gamma_{\mathfrak{u}}^{ \pm}(f, h)\right\|_{\infty} \leq\|f\|_{\infty}\|h\|_{\infty}
$$

$$
\left|\Gamma_{\mathfrak{u}}^{ \pm}(\bar{X}, \bar{X})\right| \leq \Gamma_{\mathfrak{u}}^{ \pm}(|\bar{X}|,|\bar{X}|) \leq \Gamma_{\mathfrak{u}}^{ \pm}(|X|,|X|) .
$$

We split $|X| \leq\left|\left(\mathbf{I}-\mathbf{P}_{\mathfrak{u}}\right) X\right|+\left|\mathbf{P}_{\mathfrak{u}} X\right|$. We have

$$
\Gamma_{\mathfrak{u}}^{ \pm}(|X|,|X|) \leq \Gamma_{\mathfrak{u}}^{ \pm}\left(\left|\left(\mathbf{I}-\mathbf{P}_{\mathfrak{u}}\right) X\right|,\left|\left(\mathbf{I}-\mathbf{P}_{\mathfrak{u}}\right) X\right|\right)+\Gamma_{\mathfrak{u}}^{ \pm}\left(\left|\mathbf{P}_{\mathfrak{u}} X\right|,\left|\mathbf{P}_{\mathfrak{u}} X\right|\right)+2 \tilde{\Gamma}_{\mathfrak{u}}^{ \pm}\left(\left|\left(\mathbf{I}-\mathbf{P}_{\mathfrak{u}}\right) X\right|,\left|\mathbf{P}_{\mathfrak{u}} X\right|\right),
$$

where $\tilde{\Gamma}_{\mathfrak{u}}^{ \pm}(f, g)=\frac{1}{2}\left[\Gamma_{\mathfrak{u}}^{ \pm}(f, g)+\Gamma_{\mathfrak{u}}^{ \pm}(g, f)\right]$.

Using (6.2.12) we get

$$
\varepsilon^{\frac{1}{2}}\left\|\nu^{-\frac{1}{2}} \Gamma_{\mathfrak{u}}^{ \pm}\left(\left|\mathbf{P}_{\mathfrak{u}} X\right|,\left|\mathbf{P}_{\mathfrak{u}} X\right|\right)\right\|_{2} \lesssim\left(\varepsilon^{\frac{1}{2}}\left\|\mathbf{P}_{\mathfrak{u}} X\right\|\right)_{3}\left\|\mathbf{P}_{\mathfrak{u}} X\right\|_{6} \leq[[X]]^{2} .
$$

Using (6.2.13) we get

$$
\varepsilon^{\frac{1}{2}}\left\|\nu^{-\frac{1}{2}} \Gamma_{\mathfrak{u}}^{ \pm}\left(\left|\left(\mathbf{I}-\mathbf{P}_{\mathfrak{u}}\right) X\right|,\left|\left(\mathbf{I}-\mathbf{P}_{\mathfrak{u}}\right) X\right|\right)\right\|_{2} \leq \varepsilon\left(\varepsilon^{\frac{1}{2}}\left\|\left(\mathbf{I}-\mathbf{P}_{\mathfrak{u}}\right) X\right\|\right)_{\infty}\left(\varepsilon^{-1}\left\|\left(\mathbf{I}-\mathbf{P}_{\mathfrak{u}}\right) X\right\|_{\nu}\right) \leq \varepsilon[[X]]^{2} .
$$

Similarly,

$$
\varepsilon^{\frac{1}{2}}\left\|\nu^{-\frac{1}{2}} \tilde{\Gamma}_{\mathfrak{u}}^{ \pm}\left(\left|\left(\mathbf{I}-\mathbf{P}_{\mathfrak{u}}\right) X\right|,\left|\mathbf{P}_{\mathfrak{u}} R_{n}\right|\right)\right\|_{2} \leq \varepsilon\left(\varepsilon^{\frac{1}{2}}\left\|\mathbf{P}_{\mathfrak{u}} X\right\|\right)_{\infty}\left(\varepsilon^{-1}\left\|\left(\mathbf{I}-\mathbf{P}_{\mathfrak{u}}\right) X\right\|_{\nu}\right) \leq \varepsilon[[X]]^{2} .
$$

Therefore (6.2.7) follows. Moreover, by (6.2.14), (6.2.8) follows.

Since

$$
\varepsilon^{\frac{1}{2}}\left\|\Gamma^{ \pm}\left(\left|\left(\mathbf{I}-\mathbf{P}_{\mathfrak{u}}\right) X\right|,\left|\left(\mathbf{I}-\mathbf{P}_{\mathfrak{u}}\right) X\right|\right)\right\|_{\frac{3}{2}} \leq \varepsilon^{\frac{1}{2}}\left\|\left(\mathbf{I}-\mathbf{P}_{\mathfrak{u}}\right) X\right\|_{3}^{2}
$$

and, by interpolation, $\left\|\left(\mathbf{I}-\mathbf{P}_{\mathfrak{u}}\right) X\right\|_{3} \lesssim\left\|\left(\mathbf{I}-\mathbf{P}_{\mathfrak{u}}\right) X\right\|_{\nu}^{\frac{1}{2}}\left\|\left(\mathbf{I}-\mathbf{P}_{\mathfrak{u}}\right) X\right\|_{6}^{\frac{1}{2}}$, then

$$
\varepsilon^{\frac{1}{2}}\left\|\Gamma^{ \pm}\left(\left|\left(\mathbf{I}-\mathbf{P}_{\mathfrak{u}}\right) X\right|,\left|\left(\mathbf{I}-\mathbf{P}_{\mathfrak{u}}\right) X\right|\right)\right\|_{\frac{3}{2}} \leq \varepsilon^{\frac{3}{2}}\left(\varepsilon^{-1}\left\|\left(\mathbf{I}-\mathbf{P}_{\mathfrak{u}}\right) X\right\|_{\nu} \|\right)\left\|\left(\mathbf{I}-\mathbf{P}_{\mathfrak{u}}\right) X\right\|_{6} .
$$


Since

$$
\left\|\left(\mathbf{I}-\mathbf{P}_{\mathfrak{u}}\right) X\right\|_{6} \lesssim \varepsilon^{\frac{1}{3}}\left\|\varepsilon^{-1}\left(\mathbf{I}-\mathbf{P}_{\mathfrak{u}}\right) X\right\|_{\nu}^{\frac{1}{3}} \varepsilon^{-\frac{1}{3}}\left\|\varepsilon^{\frac{1}{2}}\left(\mathbf{I}-\mathbf{P}_{\mathfrak{u}}\right) X\right\|_{\infty}^{\frac{2}{3}} \leq[[X]]
$$

we have

$$
\varepsilon^{\frac{1}{2}}\left\|\Gamma^{ \pm}\left(\left|\left(\mathbf{I}-\mathbf{P}_{\mathfrak{u}}\right) X\right|,\left|\left(\mathbf{I}-\mathbf{P}_{\mathfrak{u}}\right) X\right|\right)\right\|_{\frac{3}{2}} \leq \varepsilon^{\frac{3}{2}}[[X]]^{2}
$$

Moreover,

$$
\begin{aligned}
\varepsilon^{\frac{1}{2}}\left\|\tilde{\Gamma}^{ \pm}\left(\left|\left(\mathbf{I}-\mathbf{P}_{\mathfrak{u}}\right) X\right|,\left|\mathbf{P}_{\mathfrak{u}} X\right|\right)\right\|_{\frac{3}{2}} \leq & \left(\varepsilon^{\frac{1}{2}}\left\|\mathbf{P}_{\mathfrak{u}} X\right\|_{3}\right)\left\|\left(\mathbf{I}-\mathbf{P}_{\mathfrak{u}}\right) X\right\|_{3} \lesssim \\
& \left(\varepsilon^{\frac{1}{2}}\left\|\mathbf{P}_{\mathfrak{u}} X\right\|_{3}\right)\left\|\left(\mathbf{I}-\mathbf{P}_{\mathfrak{u}}\right) X\right\|_{\nu}^{\frac{1}{2}}\left\|\left(\mathbf{I}-\mathbf{P}_{\mathfrak{u}}\right) X\right\|_{6}^{\frac{1}{2}} \leq \varepsilon^{\frac{1}{2}}[[X]]^{2} .
\end{aligned}
$$

Finally

$$
\varepsilon^{\frac{1}{2}}\left\|\Gamma^{ \pm}\left(\left|\mathbf{P}_{\mathfrak{u}} X\right|,\left|\mathbf{P}_{\mathfrak{u}} X\right|\right)\right\|_{\frac{3}{2}} \leq\left(\varepsilon^{\frac{1}{2}}\left\|\mathbf{P}_{\mathfrak{u}} X\right\|_{3}\right)\left\|\mathbf{P}_{\mathfrak{u}} X\right\|_{3} \leq \varepsilon^{-\frac{1}{2}}\left(\varepsilon^{\frac{1}{2}}\left\|\mathbf{P}_{\mathfrak{u}} X\right\|_{3}\right)^{2} \leq \varepsilon^{-\frac{1}{2}}[[X]]^{2} .
$$

and (6.2.9) follows.

Now we are ready to bound the several terms entering in $\mathscr{M}$.

Proposition 6.9. If $|\mathfrak{u}| \ll 1$ and $\varepsilon \ll 1$ then, with

$$
\Xi_{\ell}=\sup _{0 \leq j \leq \ell}\left[\left[R_{j}\right]\right]
$$

we have

$$
\mathscr{M}\left(\varepsilon^{\frac{1}{2}} \Gamma_{\mathfrak{u}}\left(\bar{R}_{\ell}, \bar{R}_{\ell}\right)+L_{\mathfrak{u}}^{(1)} \bar{R}_{\ell}+\varepsilon^{\frac{1}{2}} \bar{A}_{\mathfrak{u}}, r_{\ell}\right) \lesssim \Xi_{\ell}^{4}+|\mathfrak{u}|^{2} \Xi_{\ell}^{2}+\varepsilon|\mathfrak{u}|^{2}+\varepsilon^{\infty} .
$$

Proof. With $g=\varepsilon^{\frac{1}{2}} \Gamma_{\mathfrak{u}}\left(\bar{R}_{\ell}, \bar{R}_{\ell}\right)+L_{\mathfrak{u}}^{(1)} \bar{R}_{\ell}+\varepsilon^{\frac{1}{2}} \bar{A}_{\mathfrak{u}}$, we have

$$
\begin{aligned}
\left\|\nu^{-\frac{1}{2}}\left(\mathbf{I}-\mathbf{P}_{\mathfrak{u}}\right) g\right\|_{2}^{2} \leq \varepsilon\left\|\nu^{-\frac{1}{2}} \Gamma_{\mathfrak{u}}\left(\bar{R}_{\ell}, \bar{R}_{\ell}\right)\right\|_{2}^{2}+\left\|\nu^{-\frac{1}{2}} L_{\mathfrak{u}}^{(1)} \bar{R}_{\ell}\right\|_{2}^{2} & +\varepsilon\left\|\nu^{-\frac{1}{2}}\left(\mathbf{I}-\mathbf{P}_{\mathfrak{u}}\right) \bar{A}_{\mathfrak{u}}\right\|_{2}^{2} \\
& \lesssim\left[\left[R_{\ell}\right]\right]^{4}+|\mathfrak{u}|^{2}\left[\left[R_{\ell}\right]\right]^{2}+\varepsilon|\mathfrak{u}|^{2},
\end{aligned}
$$

by using (6.2.7), 6.1.38), 6.1.36) and 6.1.37).

The next term in (1.44) is

$$
\begin{array}{r}
\varepsilon\left\|\nu^{-\frac{1}{2}}\left(\varepsilon^{\frac{1}{2}} \Gamma_{\mathfrak{u}}\left(\bar{R}_{\ell}, \bar{R}_{\ell}\right)+L_{\mathfrak{u}}^{(1)} \bar{R}_{\ell}+\varepsilon^{\frac{1}{2}} \bar{A}_{\mathfrak{u}}\right)\right\|_{\frac{3}{2}}^{2} \leq \varepsilon\left\|\nu^{-\frac{1}{2}} \varepsilon^{\frac{1}{2}} \Gamma_{\mathfrak{u}}\left(\bar{R}_{\ell}, \bar{R}_{\ell}\right)\right\|_{\frac{3}{2}}^{2}+\varepsilon\left\|\nu^{-\frac{1}{2}} L_{\mathfrak{u}}^{(1)} \bar{R}_{\ell}\right\|_{\frac{3}{2}}^{2} \\
+\varepsilon\left\|\nu^{-\frac{1}{2}} \varepsilon^{\frac{1}{2}} \bar{A}_{\mathfrak{u}}\right\|_{\frac{3}{2}}^{2} \lesssim\left[\left[R_{\ell}\right]\right]^{4}+\varepsilon|\mathfrak{u}|\left[\left[R_{\ell}\right]\right]^{2}+\varepsilon^{4}|\mathfrak{u}|^{2},
\end{array}
$$

by using (6.2.9), (6.1.38), (6.1.36) and (6.1.37).

Then we have

$$
\begin{aligned}
& \varepsilon^{3} \|\langle v\rangle^{-1} w {\left[\varepsilon^{\frac{1}{2}} \Gamma_{\mathfrak{u}}\left(\bar{R}_{\ell}, \bar{R}_{\ell}\right)+L_{\mathfrak{u}}^{(1)} \bar{R}_{\ell}+\varepsilon^{\frac{1}{2}} \bar{A}_{\mathfrak{u}}\right] \|_{\infty}^{2} \leq } \\
&\left.\varepsilon^{3}\left\|\langle v\rangle^{-1} w \varepsilon^{\frac{1}{2}} \Gamma_{\mathfrak{u}}\left(\bar{R}_{\ell}, \bar{R}_{\ell}\right)\right\|_{\infty}^{2}+\varepsilon^{3}\left\|\langle v\rangle^{-1} w L_{\mathfrak{u}}^{(1)} \bar{R}_{\ell}\right\|_{\infty}^{2}+\varepsilon^{3} \| \varepsilon^{\frac{1}{2}}\langle v\rangle^{-1} w \bar{A}_{\mathfrak{u}}\right] \|_{\infty}^{2} \\
& \quad \lesssim \varepsilon^{2}\left[\left[R_{\ell}\right]\right]^{4}+\varepsilon^{2}|\mathfrak{u}|\left[\left[R_{\ell}\right]\right]^{2}+\varepsilon^{2}|\mathfrak{u}|^{2},
\end{aligned}
$$

by using (6.2.8), 6.1.39), 6.1.36) and 6.1.37).

Since $\mathbf{P}_{\mathfrak{u}} g=\varepsilon^{1 / 2} \mathbf{P}_{\mathfrak{u}} \bar{A}_{\mathfrak{u}}$, the term $\left\|\mathbf{P}_{\mathfrak{u}} g\right\|_{2}^{2}+\varepsilon^{-2}|\mathfrak{u}|^{2}\left\|\mathbf{P}_{\mathfrak{u}} g\right\|_{\frac{6}{5}}^{2}$ in (1.44) is bounded by $\varepsilon^{\infty}$ using (6.1.36). Next we bound

$$
\left\|z_{\gamma}\left(r_{\ell}\right)\right\|_{2}^{2} \lesssim\left\|z_{\gamma}(\bar{r})\right\|_{2}^{2}+\varepsilon^{-1}\left\|z_{\gamma}\left(P_{\gamma}^{\mathfrak{u}} \tilde{R}_{\ell}\right)\right\|_{2}^{2}
$$

The first term is bounded by $\varepsilon^{\infty}$ using (6.1.21). Moreover, by (6.1.28),

$$
\begin{aligned}
\left(\varepsilon^{\frac{1}{2}-2 \sigma}|\mathfrak{u}|^{-2+2 \varrho}+|\mathfrak{u}|^{-1} \varepsilon^{-1}\right) & \varepsilon^{-1}\left\|z_{\gamma}\left(P_{\gamma}^{\mathfrak{u}} \tilde{R}_{\ell}\right)\right\|_{2}^{2} \\
& \leq \varepsilon^{-2}|\mathfrak{u}|^{-1}\left[\varepsilon\left(|\mathfrak{u}|+\varepsilon^{\frac{1}{2}}\left\|w R_{\ell}\right\|_{\infty}\right)\right]^{2(1+\beta)}\left|R_{\ell}\right|_{2,+}^{2} \\
& \leq \varepsilon^{2 \beta}|\mathfrak{u}|^{-1}\left(|\mathfrak{u}|+\varepsilon^{\frac{1}{2}}\left\|w R_{\ell}\right\|_{\infty}\right)^{2(1+\beta)}\left|R_{\ell}\right|_{2,+}^{2}
\end{aligned}
$$


To bound $\left|R_{\ell}\right|_{2,+}$ we use Lemma 2.2 and (6.2.1) with $\ell$ replaced by $\ell-1$ to obtain

$$
\begin{array}{r}
\left|R_{\ell}\right|_{2,+}^{2} \lesssim\left\|\mathbf{P}_{\mathfrak{u}} R_{\ell}\right\|_{6}^{2}+\left(\varepsilon^{-1}\left\|\left(\mathbf{I}-\mathbf{P}_{\mathfrak{u}}\right) R_{\ell}\right\|_{\nu}\right)^{2}+\varepsilon\left\|\nu^{-\frac{1}{2}} \Gamma_{\mathfrak{u}}\left(\bar{R}_{\ell-1}, \bar{R}_{\ell-1}\right)\right\|_{2}+\left\|\nu^{-\frac{1}{2}} L_{\mathfrak{u}}^{(1)} \bar{R}_{\ell-1}\right\|_{2}^{2} \\
+\varepsilon\left\|\nu^{-\frac{1}{2}} \bar{A}_{\mathfrak{u}}\right\|_{2}^{2} \lesssim\left[\left[R_{\ell}\right]\right]^{2}+\Xi_{\ell}^{4}+|\mathfrak{u}|^{2} \Xi_{\ell}^{2}+\varepsilon|\mathfrak{u}|^{2}, \quad(6
\end{array}
$$

by using (6.2.7), (6.1.38), 6.1.36) and (6.1.37). Hence, since $\Xi<\vartheta<1$, for $\varepsilon \ll 1$ we have

$$
\begin{aligned}
\left.\left(\varepsilon^{-2 \sigma}|\mathfrak{u}|^{-2+2 \varrho}+|\mathfrak{u}|^{-1} \varepsilon^{-1}\right) \varepsilon^{-1} \| r_{\ell}\right) \|_{2}^{2} & \\
\lesssim \varepsilon^{\infty}+\varepsilon^{2 \beta}|\mathfrak{u}|^{-1}\left(|\mathfrak{u}|+\left[\left[R_{\ell}\right]\right]\right)^{2(1+\beta)}\left\{\left[\left[R_{\ell}\right]\right]^{2}\right. & \left.+\Xi_{\ell}^{4}+|\mathfrak{u}|^{2} \Xi_{\ell}^{2}+|\mathfrak{u}|^{2}\right\} \\
& \lesssim \Xi_{\ell}^{4}+|\mathfrak{u}|^{2} \Xi_{\ell}^{2}+\varepsilon|\mathfrak{u}|^{2}+\varepsilon^{\infty},
\end{aligned}
$$

The terms $|r|_{2,-}^{2}$ is treated in a similar way. As for $\varepsilon|w r|_{\infty}$ we proceed as before using (6.1.29), (6.2.8), 6.1.39) and 6.1.36 and 6.1.37).

Collecting the estimates, since $\varepsilon<1$, we conclude that

$$
\mathscr{M}\left(\varepsilon^{\frac{1}{2}} \Gamma_{\mathfrak{u}}\left(\bar{R}_{\ell}, \bar{R}_{\ell}\right)+L_{\mathfrak{u}}^{(1)} \bar{R}_{\ell}+\varepsilon^{\frac{1}{2}} \bar{A}_{\mathfrak{u}}, r_{\ell}\right) \lesssim \Xi_{\ell}^{4}+|\mathfrak{u}|^{2} \Xi_{\ell}^{2}+\varepsilon|\mathfrak{u}|^{2}+\varepsilon^{\infty}
$$

Since $\Xi_{\ell} \leq \vartheta$, from (1.43) we obtain

$$
\left[\left[R_{\ell+1}\right]\right]^{2} \leq \vartheta^{2}\left[\vartheta^{2}+c_{0}^{2} \vartheta^{2}+c_{0}^{2}+\varepsilon^{\infty} \vartheta^{-2}\right]<\vartheta^{2},
$$

provided that

$$
\vartheta^{2}+c_{0}^{2} \vartheta^{2}+c_{0}^{2}+\varepsilon^{\infty} \vartheta^{-2}<1 .
$$

This is verified if $\vartheta \ll 1, \varepsilon \ll 1, c_{0} \ll 1$.

The same arguments prove (6.2.6), by using (6.1.13) and (6.1.14). The sequence $\left\{R_{\ell}\right\}$ thus converges strongly to $R$ such that $[[R]]<\vartheta$. It is standard to check that $R$ solves (6.1.22). Since convergence in $[[\cdot]]$ implies pointwise convergence, by (6.1.14) it follows that $R$ satisfies (6.1.23).

Therefore $F=\mu_{\mathfrak{u}}+\varepsilon \mathscr{Q}+\varepsilon^{\frac{3}{2}} R$ solves the problem (6.1.1), 6.1.2) and hence it is positive by construction. Moreover, it is in $L^{\infty}$, even if not uniformly bounded in $\varepsilon$. We can use Proposition 6.1 to conclude that it is also solution to the original problem (1.1), with boundary condition (1.8) and condition at infinity (1.12). The same estimates also prove uniqueness in the larger space because we can drop the assumption $\beta>0$ which was used before only to deal with terms appearing in the modified problem (6.1.1), (6.1.2).

\section{ApPEndiX A. Bounds ON THE VELOCITY FIELD}

Proposition A.1. If $|\mathfrak{u}|$ is sufficiently small, then the solution to the problem

$$
\begin{aligned}
& U \cdot \nabla U+\nabla p=\Delta U, \quad \nabla \cdot U=0, \quad \text { in } \Omega^{c} \\
& \lim _{|x| \rightarrow \infty} U=\mathfrak{u},\left.\quad U\right|_{\partial \Omega}=0,
\end{aligned}
$$

is such that

$$
\|U-\mathfrak{u}\|_{p} \lesssim|\mathfrak{u}|, \quad \text { for any } p \geq 3 .
$$

Proof. We first construct $w(x)$ such that $\nabla \cdot w(x)=0, \lim _{|x| \rightarrow \infty} w(x)=\mathfrak{u}$, and $\left.w(x)\right|_{\partial \Omega}=0$, with $|w(x)-\mathfrak{u}|=0$ for $x$ sufficiently large. In fact (see [19]) we can choose

$$
w=\mathfrak{u}-\operatorname{curl}\left[\chi(d(x, \partial \Omega))\left(\mathfrak{u}_{2} x_{3}, \mathfrak{u}_{3} x_{1}, \mathfrak{u}_{1} x_{2}\right)\right]
$$


where $\chi(z)$ is smooth with $\chi(z)=1$ for $x<\frac{1}{2}$ and $\chi(z)=0$ for $z \geq 1$. By construction $\nabla \cdot w=0$. Moreover, we have

$$
\begin{aligned}
& \operatorname{curl}\left[\chi(d(x, \partial \Omega))\left(\mathfrak{u}_{2} x_{3}, \mathfrak{u}_{3} x_{1}, \mathfrak{u}_{1} x_{2}\right)\right]= \\
& \chi^{\prime}(d(x, \partial \Omega)) \nabla_{x} d(x, \partial \Omega)\left(\mathfrak{u}_{2} x_{3}, \mathfrak{u}_{3} x_{1}, \mathfrak{u}_{1} x_{2}\right)+\chi(d(x, \partial \Omega)) \mathfrak{u}= \begin{cases}\mathfrak{u} & x \in \partial \Omega, \\
0 & d(x, \partial \Omega)>1 .\end{cases}
\end{aligned}
$$

Clearly $w-\mathfrak{u}$ is compactly supported and $\|w\|_{W^{s, p}} \lesssim|\mathfrak{u}|$ for any $p \geq 1$ and any $s \geq 0$. We then seek for $U=w+v$, with $v$ such that

$$
\begin{aligned}
& w \cdot \nabla v-\Delta v+\nabla p=-(w+v) \cdot \nabla w+\Delta w-v \cdot \nabla v \\
& \lim _{|x| \rightarrow \infty} v=\left.0 \quad v\right|_{\partial \Omega}=0 .
\end{aligned}
$$

We construct the approximating sequence solving

$$
\begin{aligned}
& w \cdot \nabla v^{\ell}-\Delta v^{\ell}+\nabla p^{\ell}=-\left(w+v^{\ell}\right) \cdot \nabla w+\Delta w-v^{\ell-1} \cdot \nabla v^{\ell} \\
& \lim _{|x| \rightarrow \infty} v^{\ell}=\left.0 \quad v^{\ell}\right|_{\partial \Omega=0}=0,
\end{aligned}
$$

for $\ell \geq 1$ and $v^{0}=0$.

Step 1. By energy estimate and weak solution theory, we can show there is a solution $v$ to (A.4), (A.5), unique for $|\mathfrak{u}| \ll 1$, which is the weak limit of $\left\{v^{\ell}\right\}$ and for any $\ell$

$$
\left\|\nabla v^{\ell}\right\|_{L^{2}}+\left\|v^{\ell}\right\|_{L^{6}} \lesssim|\mathfrak{u}| \text {. }
$$

Step 2. We now show that $v \in L^{3}$ and $\|v\|_{L^{3}} \lesssim|\mathfrak{u}|$. Using $\partial_{j} \mathfrak{u}=0, \nabla \cdot v^{\ell}=0$ and $\nabla \cdot w=0$, we write the $i$-th component of (A.6) as

$$
\sum_{j=1}^{3}\left[\mathfrak{u}_{j} \partial_{j} v_{i}^{\ell}-\partial_{j}^{2} v_{i}^{\ell}\right]+\partial_{i} p^{\ell}=-\sum_{j=1}^{3} \partial_{j}\left[-\partial_{j} w_{i}+\left(w_{j}-\mathfrak{u}_{j}\right) v_{i}^{\ell}+v_{j}^{\ell}\left(w_{i}-\mathfrak{u}_{i}\right)+w_{j} w_{i}+v_{j}^{\ell-1} v_{i}^{\ell}\right] .
$$

In Fourier space, we have (using the Leray Projector $\Pi$, and $k \cdot \hat{v}(k)=0$ ):

$$
\widehat{\partial_{m} v_{i}^{\ell}}=\sum_{j=1}^{3} \frac{k_{m} k_{j}}{|k|^{2}+i \mathfrak{u} \cdot k} \hat{\Pi} \mathcal{F}\left\{-\partial_{j} w_{i}+\left(w_{j}-\mathfrak{u}_{j}\right) v_{i}^{\ell}+v_{j}^{\ell}\left(w_{i}-\mathfrak{u}_{i}\right)+w_{j} w_{i}+v_{j}^{\ell-1} v_{i}^{\ell}\right\} .
$$

We have

$$
\left|\partial_{k}^{l} \frac{k_{m} k_{j}}{|k|^{2}+i \mathfrak{u} \cdot k} \hat{\Pi}\right| \leq \frac{1}{|k|^{l}},
$$

independent of $\mathfrak{u}$. Hence we can use the Mihlin-Hormander theorem. Therefore, by Sobolev embedding in $3 \mathrm{D}\left(W^{1, \frac{3}{2}} \subset L^{3}\right)$ and the compact support of $w-\mathfrak{u}$, we obtain

$$
\begin{aligned}
\left\|v^{\ell}\right\|_{L^{3}} & \leq \sup _{m}\left\|\partial_{m} v^{\ell}\right\|_{\frac{3}{2}} \\
& \leq\left\|-\partial_{j} w_{i}+\left(w_{j}-\mathfrak{u}_{j}\right) v_{i}^{\ell}+v_{j}^{\ell}\left(w_{i}-\mathfrak{u}_{i}\right)+w_{j} w_{i}+v_{j}^{\ell-1} v_{i}^{\ell}\right\|_{L^{\frac{3}{2}}} \\
& \lesssim|\mathfrak{u}|\left(1+\left\|v^{\ell}\right\|_{6}\right)+\left\|\left|v ^ { \ell - 1 } \left\|v ^ { \ell } \left|\left\|_{L^{\frac{3}{2}}} \lesssim|\mathfrak{u}|+\right\| v^{\ell}\left\|_{L^{3}}\right\| v^{\ell-1} \|_{L^{3}} .\right.\right.\right.\right.
\end{aligned}
$$

Therefore, if we assume the recurrence hypothesis $\sup _{0 \leq m \leq \ell-1}\left\|v^{m}\right\|_{L^{3}} \leq C|\mathfrak{u}|$, by choosing $|\mathfrak{u}| \ll 1$ we obtain

and the limit satisfies $\|v\|_{L^{3}} \leq C|\mathfrak{u}|$.

$$
\left\|v^{\ell}\right\|_{L^{3}} \leq C|\mathfrak{u}|
$$

Step 3. By differentiating the equation, from the energy inequality for the derivative we obtain $\overline{\|D v\|_{6}} \lesssim|\mathfrak{u}|$ and hence $\|v\|_{\infty} \lesssim|\mathfrak{u}|$. By interpolation we conclude (A.3).

Acknowledgments Yan Guo's research is supported in part by NSF grant 1611695, Chinese NSF grant 10828103, as well as a Simon Fellowship. R. Marra's research is partially supported by MIUR-Prin. We are very grateful for extensive and constructive comments from the referees, which help us to improve the presentation of the paper. We thank Junhwa Jung for pointing out some omissions. 


\section{REFERENCES}

[1] Arkeryd L.; Nouri A.: On a Taylor-Couette Type Bifurcation for the Stationary Nonlinear Boltzmann Equation Jour. Stat. Phys., 124, pp. 401-443 (2006)

[2] Babenko, K.I.: On Stationary Solutions of the Problem of Flow Past a Body of a Viscous Incompressible Fluid, Mat. Sb., 91 (133), 3-27 (1973); English Transl.: Math. SSSR Sbornik, 20 1973, 1-25 (1973)

[3] C. Bardos; F. Golse; D. Levermore: Sur les limites asymptotiques 41Acad. Sci. Paris Sér. I Math., 309, $727-732(1989)$

[4] C. Bardos; S. Ukai: The classical incompressible Navier-Stokes limit of the Boltzmann equation, Math. Mod. Meth. Appl. S., vol.1, p 235 (1991)

[5] Bobylev A. V.; Mossberg E.: On Some Properties of Linear and Linearized Boltzmann Collision Oerators for Hard Spheres Kinetic and Related Models, 1, pp. 521-555 (2008)

[6] C. Cercignani, Mathematical methods in Kinetic Theory, Plenum Press, New York 1969

[7] C. Cercignani; R. Illner; M. Pulvirenti: The mathematical theory of dilute gases, Springer-Verlag (1994)

[8] A. De Masi; R. Esposito; J. L. Lebowitz: Incompressible Navier-Stokes and Euler Limits of the Boltzmann Equation, Comm. Pure and Appl. Math., 42, 1189-1214 (1989)

[9] R. Esposito; Y. Guo; C. Kim; R. Marra: Non-Isothermal Boundary in the Boltzmann Theory and Fourier Law, Comm. Math. Phys. 323, 177-239 (2013)

[10] R. Esposito; Y. Guo; C. Kim; R. Marra: Stationary solutions to the Boltzmann equation in the Hydrodynamic limit, Annals of PDE, 4 pp. 1-119 (2018), DOI 10.1007/s40818-017-0037-5; ArXiv, 1502.05324v3 (2016)

[11] L. C. Evans: Partial Differential Equations, Graduate Studies in Mathematics, vol. 19 American Mathematical Society (1998)

[12] Finn, R.: Estimates at Infinity for Stationary Solutions of the Navier-Stokes Equations, Bull. Math. Soc. Sci. Math. Phys. R. P. Roumaine, 3 (51) 387-418 (1959)

[13] Galdi G. P.: An Introduction to the Mathematical Theory of the Navier-Stokes Equations Springer (2011)

[14] F. Golse; L. Saint-Raymond: The Navier-Stokes limit of the Boltzmann equation for bounded collision kernels, Invent. Math. 155, 81-161 (2004)

[15] Y. Guo: Boltzmann diffusive limit beyond the Navier-Stokes approximation. Comm. Pure and Appl. Math. 59, 626-687 (2006)

[16] Y. Guo; J. Jang: Global Hilbert expansion for the Vlasov-Poisson-Boltzmann system. Comm. Math. Phys. 299, 469-501 (2010)

[17] Y. Guo; J. Jang; N. Jiang: Acoustic limit for the Boltzmann equation in optimal scaling. Comm. Pure Appl. Math. 63, 337-361 (2010)

[18] Hörmander L.: Estimates for translation invariant operators in $L^{p}$ spaces. Acta Math. 104, 93-140 (1960).

[19] O. Ladyzhenskaya: Mathematical Theory of Viscous Incompressible Flow, 2th. ed., Gordon and Breach (1969)

[20] Lamb, H.: Hydrodynamics (Sixth ed.). Dover Publications. (1945)

[21] Leoni G.: A First Course in Sobolev Spaces, AMS Grad. Stud. in Math. (2009)

[22] Leray, J.: Étude de Diverses Équations Integrales non Linéaires et de Quelques Problèmes que Pose l' Hydrodynamique, J. Math. Pures Appl., 12, 1-82 (1933)

[23] Lions, P.-L.; Masmoudi, N. : From the Boltzmann equations to the equations of incompressible fluid mechanics. I, II. Arch. Ration. Mech. Anal. 158, no. 3, 173-193, 195-211 (2001)

[24] N. Maslova: Nonlinear Evolution Equations, World Scientific (1993)

[25] Masmoudi, N. ; Saint-Raymond, L. : From the Boltzmann equation to the Stokes-Fourier system in a bounded domain. Comm. Pure Appl. Math. 56, no. 9, 1263-1293 (2003)

[26] Mihlin S. G.: On the multipliers of Fourier integrals (Russian). Dokl. Akad. Nauk SSSR 12, 143-155 (1957).

[27] Oseen, C. W.: Über die Stokes'sche formel, und über eine verwandte Aufgabe in der Hydrodynamik, Arkiv för matematik, astronomi och fysik, vi (29) (1910)

[28] Saint-Raymond, L.: Hydrodynamic Limits of the Boltzmann Equation, Springer Lecture Notes in Mathematics, (2009)

[29] Thomson, W.: On ship waves, Institution of Mechanical Engineers, Proceedings, 38 : 409-434 (1887)

[30] Ukai S.; Asano K.: Steady solutions of the Boltzmann equation for a gas flow past an obstacle. I. Existence. Arch. Rational Mech. Anal. 84, 249-291 (1983).

[31] Ukai S.; Asano K.: Steady solutions of the Boltzmann equation for a gas flow past an obstacle. II. Stability. Publ. Res. Inst. Math. Sci. 22, 1035-1062 (1986)

[32] Ukai S.; Yang T.; Zhao H.: Stationary solutions to the exterior problems for the Boltzmann equation. I. Existence. Discrete Contin. Dyn. Syst. 23, 495-520 (2009)

[33] Ukai S.; Yang T.; Zhao H.: Exterior Problem for the Boltzmann equationwith Temperature Difference. Commun. in Pure and Appl. Analysis, 8, pp 473-491 (2009)

[34] L. Wu; Y. Guo: Geometric Correction for Diffusive Expansion of Steady Neutron Transport Equation, arXiv:1404.2583, in Comm. Math. Phys. 336 1473-1533 (2015) 